\title{
Visual Spatial Attention to Multiple Locations at Once : The Jury Is Still Out
}

Citation for published version (APA):

Jans, B., Peters, J. C., \& de Weerd, P. (2010). Visual Spatial Attention to Multiple Locations at Once : The Jury Is Still Out. Psychological Review, 117(2), 637-684. https://doi.org/10.1037/a0019082

Document status and date:

Published: 01/01/2010

DOI:

10.1037/a0019082

Document Version:

Publisher's PDF, also known as Version of record

Document license:

Taverne

Please check the document version of this publication:

- A submitted manuscript is the version of the article upon submission and before peer-review. There can be important differences between the submitted version and the official published version of record.

People interested in the research are advised to contact the author for the final version of the publication, or visit the DOI to the publisher's website.

- The final author version and the galley proof are versions of the publication after peer review.

- The final published version features the final layout of the paper including the volume, issue and page numbers.

Link to publication

\footnotetext{
General rights rights.

- You may freely distribute the URL identifying the publication in the public portal. please follow below link for the End User Agreement:

www.umlib.nl/taverne-license

Take down policy

If you believe that this document breaches copyright please contact us at:

repository@maastrichtuniversity.nl

providing details and we will investigate your claim.
}

Copyright and moral rights for the publications made accessible in the public portal are retained by the authors and/or other copyright owners and it is a condition of accessing publications that users recognise and abide by the legal requirements associated with these

- Users may download and print one copy of any publication from the public portal for the purpose of private study or research.

- You may not further distribute the material or use it for any profit-making activity or commercial gain

If the publication is distributed under the terms of Article $25 \mathrm{fa}$ of the Dutch Copyright Act, indicated by the "Taverne" license above, 
See discussions, stats, and author profiles for this publication at: https://www.researchgate.net/publication/44569227

\section{Visual Spatial Attention to Multiple Locations at Once: The Jury Is Still Out}

Article in Psychological Review · April 2010

DOl: 10.1037/00019082 · Source: PubMed

CITATIONS

112

3 authors:

Bert Jans

Maastricht University

8 PUBLICATIONS 143 CITATIONS

SEE PROFILE

C. Peter De Weerd

Maastricht University

149 PUBlications 6,475 CITATIONS

SEE PROFILE

Some of the authors of this publication are also working on these related projects:

Project The role of spatial frequencies in neural face processing View project

Project Memory of time View project
READS

1,471

Judith C Peters

Maastricht University

67 PUBLICATIONS 1,878 CITATIONS

SEE PROFILE 


\title{
Visual Spatial Attention to Multiple Locations at Once: The Jury Is Still Out
}

\author{
Bert Jans \\ Maastricht University
}

\author{
Judith C. Peters \\ Maastricht University and Netherlands Institute for \\ Neuroscience, Amsterdam, the Netherlands
}

\author{
Peter De Weerd \\ Maastricht University and Donders Institute for Brain, Cognition and Behaviour, Nijmegen, the Netherlands
}

\begin{abstract}
Although in traditional attention research the focus of visual spatial attention has been considered as indivisible, many studies in the last 15 years have claimed the contrary. These studies suggest that humans can direct their attention simultaneously to multiple noncontiguous regions of the visual field upon mere instruction. The notion that spatial attention can easily be split is counterintuitive in the light of current neurocognitive models of attention. We examined studies on divided attention against 4 methodological criteria that should be satisfied in order to convincingly demonstrate divided attention, and we found no studies in the current literature that pass this test. On the basis of current theories of attention, we argue that dividing attention may not be easily achievable by naive human observers and that, instead, it is a skill that may be acquired only through training.
\end{abstract}

Keywords: divided spatial attention, experimental design criteria, theoretical constraints, divided spatial attention in dual tasks, divided spatial attention as part of expert task performance

In an important article, McCormick, Klein, and Johnston (1998) evaluated the evidence from visual divided attention studies and concluded that "most of the evidence favors the unified model" ( $\mathrm{p}$. 350 ). According to the unified model, attentional modulation is confined to a single, indivisible focal region in the visual field. A decade later, Adamo, Pun, Pratt, and Ferber (2008) introduced their article with the statement that "there is abundant evidence showing that observers can deploy attention to separate locations in space" (p. 296). What has happened in recent years to explain this drastic change in zeitgeist?

Bert Jans, Department of Cognitive Neuroscience, Maastricht University, Maastricht, the Netherlands; Judith C. Peters, Department of Cognitive Neuroscience, Maastricht University, and Department of Neuroimaging and Neuromodeling, Netherlands Institute for Neuroscience, Amsterdam, the Netherlands; Peter De Weerd, Department of Cognitive Neuroscience, Maastricht University, and Donders Institute for Brain, Cognition and Behaviour, Radboud University Nijmegen, Nijmegen, the Netherlands.

The writing of this article was supported in part by an open competition grant (400-04-036) and a VICI grant (015-056-604) from the Netherlands Organization for Scientific Research (NWO) to Peter De Weerd and an NWO project (402-01-632) supporting Judith C. Peters. The first version was written by Bert Jans, and subsequent versions by Peter De Weerd with contributions from Judith C. Peters and Bert Jans. The three authors may claim first authorship of this work. We are grateful for feedback in various phases of the article by Pascal Fries, Claudia Schreiner, and Saskia Ranson. In addition, we thank Claus Bundesen, Kyle Cave, and Peter McCormick for their insightful comments and suggestions.

Correspondence concerning this article should be addressed to Peter De Weerd, Faculty of Psychology and Neuroscience, Department of Cognitive Neuroscience, Maastricht University, PO Box 616, Maastricht 6200 MD, the Netherlands. E-mail: p.deweerd@maastrichtuniversity.nl
During the last 15 years, an increasing number of studies have claimed that the focus of attention can be divided over multiple noncontiguous locations in the visual field. This assertion is supported by what appears to be converging evidence from behavioral, functional magnetic resonance imaging (fMRI), and electrophysiological studies in humans. These studies all suggest that human observers can simultaneously attend to and identify stimuli presented at the same time in multiple locations. Furthermore, human observers typically appear to be able to divide attention whenever instructed to do so, without preceding training. This suggests a tremendous flexibility in the way attention is allocated and argues against the unitary nature of the attentional focus, which is at the heart of serial models of attention. For these reasons, findings supporting a division of the attentional focus have received a lot of interest. How well do these findings hold up, however, in the face of closer scrutiny?

Importantly, the question whether attention can be divided and allocated in parallel pertains only to spatial attention (to locations in the visual field). Spatial attention should be distinguished from feature-related attention (to aspects of objects such as their orientation and color; for review, see Maunsell \& Treue, 2006), which is well known to operate in parallel across the visual field (Bichot, Rossi, \& Desimone, 2005; Hopf et al., 2000; Maunsell \& Treue, 2006; Motter, 1994a, 1994b; H. J. Müller \& Ebeling, 2008) Because of the distinction between the two forms of attention, the parallel processing of object features does not imply that spatial attention operates in parallel as well. Evidence from several disciplines supports the idea that the mechanisms underlying spatial and feature-related attention are different. For example, human magnetoencephalography experiments (Hopf, Boelmans, Schoenfeld, Luck, \& Heinze, 2004) and neurophysiological experiments 
in monkeys (Bichot et al., 2005) have demonstrated that during visual search, feature-based attention precedes spatial attention. By contrast, in a match-to-sample task, a neurophysiological study in monkeys indicated that parietal activity leads temporal activity during attention to a single location, suggesting a precedence of spatial attention (Saalmann, Pigarev, \& Vidyasagar, 2007). Furthermore, feature-based attention can operate outside the focus of spatial attention (e.g., Motter, 1993, 1994a, 1994b; Treue \& Martínez Trujillo, 1999), and interactions between visual areas and parietofrontal areas that control attentional allocation are different for these two types of attention (Schenkluhn, Ruff, Heinen, \& Chambers, 2008; F. A. Wilson, Scalaidhe, \& Goldman-Rakic, 1993). Many models of attention (Desimone \& Duncan, 1995; Duncan, 1984; Kröse \& Julesz, 1989; Mordkoff, Yantis, \& Egeth, 1990; Shiffrin \& Schneider, 1977; Treisman \& Gormican, 1988; Treisman \& Sato, 1990; Wolfe, 1994) are based on the differential contributions of feature-related and spatial attention.

Despite significant conceptual differences among theories, they commonly distinguish two major steps in attentional processing when multiple stimuli are presented simultaneously. It is proposed that early processing of stimuli in a visual scene occurs in parallel with no or minor capacity limits (Joseph, Chun, \& Nakayama, 1997; Sagi \& Julesz, 1985) and is followed by late processing to achieve full analysis of a selected, relevant object. Late processing involves the spatial restriction of attention to the selected object (Bichot et al., 2005; Brefczynski \& DeYoe, 1999; Chelazzi, Miller, Duncan, \& Desimone, 1993; Kastner, Pinsk, De Weerd, Desimone, \& Ungerleider, 1999; Reynolds, Chelazzi, \& Desimone, 1999; Somers, Dale, Seiffert, \& Tootell, 1999; Treue \& Maunsell, 1999) and is subject to severe capacity limitations (Broadbent, 1958; Egeth, 1977; C. W. Eriksen \& St. James, 1986; Kahneman, 1973; Shaw \& Shaw, 1977). The focused analysis of a selected object is what we term spatial attention, and the question whether spatial attention can be divided comes down to whether limited attentional capacity during full processing of stimuli can be allocated in parallel to separate spatial locations. Here other interpretations of the term spatial attention related to the encoding of space itself, including the encoding of spatial relationships among objects or of objects relative to an observer in different spatial reference frames (e.g., Andersen, Snyder, Bradley, \& Xing, 1997; Colby \& Goldberg, 1999; Graziano, 2001; Olson, 2003), will not be considered.

In the analysis of divided attention studies that we offer, we take as a null hypothesis the traditional view that spatial attention cannot be divided (Broadbent, 1982; Downing \& Pinker, 1985; B. A. Eriksen \& Eriksen, 1974; C. W. Eriksen \& St. James, 1986; McCormick \& Klein, 1990; McCormick et al., 1998; Miller, 1988; Pan \& Eriksen, 1993; Posner, 1980; Posner, Snyder, \& Davidson, 1980; Thornton \& Gilden, 2007). Recent human psychophysical evidence (Thornton \& Gilden, 2007; VanRullen, Carlson, \& Cavanagh, 2007), event-related potential (ERP) evidence in humans (Woodman \& Luck, 1999, 2003), and neurophysiological evidence in monkeys (e.g., Bichot et al., 2005; Chelazzi et al., 1993) support the idea that the default mode of attentional processing is to allocate resources in a unified focus during target selection.

There are three lines of research and theoretical thinking that underscore the idea that divided attention is not a self-evident feature of the attentional system and that it is therefore reasonable to take the hypothesis of unified attention as a null hypothesis First, spatial attention is closely related to the selection of a target object for action, and motor actions are usually directed serially toward single objects in single locations, rather than simultaneously to several objects in multiple locations. A prime example is the foveating of individual objects, which almost naturally leads to the concept of a focus of attention "illuminating" relevant objects in a scene one at a time (B. A. Eriksen \& Eriksen, 1974). In the same vein, the classical spatial cuing experiments from Posner and colleagues (Posner \& Cohen, 1984; Posner et al., 1980) have been interpreted as evidence for a serially moving "spotlight" of attention (Posner et al., 1980). The study from Bichot et al. (2005) strongly suggests that the selection of a target for action involves an increase in attentional enhancement at the target location only. Although mechanisms of spatial attention and motor planning (intention) can be distinguished (Andersen \& Buneo, 2002; Andersen et al., 1997; Juan, Shorter-Jacobi, \& Schall, 2004; Thompson, Biscoe, \& Sato, 2005), spatial attention and mechanisms related to motor planning and preparation are closely related (Sheliga, Riggio, \& Rizzolatti, 1994) and closely linked within the parietal and premotor cortices (Andersen \& Buneo, 2002; Colby \& Goldberg, 1999; Corbetta, 1998; T. Moore \& Armstrong, 2003). It would be surprising that mechanisms of covert attention would routinely maintain separate foci of heightened processing given that motor action is essentially serial. Even when more effectors are involved (e.g., eye and hand movements), their action is usually coordinated and directed at the same location (see, e.g., Johansson, Westling, Bäckström, \& Flanagan, 2001). Hence, the link between spatial attention and motor action and the fact that motor action is directed usually to a single location argue against the idea that spatial attention can easily be divided, in an ondemand, flexible manner. However, in some conditions, multiple sources of information may be relevant simultaneously. This may happen, for example, while threading a needle, hitting a nail with a hammer, or monitoring information from multiple instruments on an instrument panel in an aircraft cockpit. In these situations, it can be argued that attention will be split to process relevant, disparate parts of the retinal image simultaneously. However, the simultaneous relevance of multiple locations does not imply that they are processed with split attention. Moreover, attending to multiple locations simultaneously (if possible at all) is likely to come at a significant cost, making it unlikely to be a mechanism of choice in daily life conditions. We suggest that the default mode of processing is to shift attention serially between relevant locations and that only in special conditions (e.g., time pressure) this mechanism might fail, which then opens the unanswered question whether in those conditions information might be processed (at a cost) by dividing spatial attention.

Second, an influential school of thought suggests that the allocation of spatial attention is controlled by a saliency map that encodes relevance in a single location independent of specific features of the object (e.g., Koch \& Ullman, 1985), and the lateral intraparietal area has been proposed as a potential neuronal substrate of such a map (Gottlieb, Kusunoki, \& Goldberg, 1998; Kusunoki, Gottlieb, \& Goldberg, 2000). The activity in the saliency map is thought to modulate processing in retinotopically organized areas in the temporal lobe that analyze objects (Desimone \& Duncan, 1995). Other brain regions that could function as a saliency map include the frontal eye fields (T. Moore \& Arm- 
strong, 2003; T. Moore \& Fallah, 2004), the pulvinar (Desimone, Wessinger, Thomas, \& Schneider, 1990; Petersen, Robinson, \& Morris, 1987), and the superior colliculus (Wurtz \& Goldberg, 1972). To date, however, there is no evidence that the lateral intraparietal area or any other structure involved in the control of spatial attention could sustain two stable, spatially segregated regions of attentional enhancement. Although the absence of that evidence does not exclude the possibility of spatially divided attention, the absence of empirical support for this hypothesis with arguably the most direct method (neurophysiology) makes a conclusion of divided attention difficult in human experiments that use less direct methods (fMRI, ERPs, psychophysics), especially if experimental conditions were not designed to exclude as much as possible the unified attention hypothesis.

Third, the idea of split spatial attention cannot easily be reconciled with the biased competition model of attention (Desimone \& Duncan, 1995), an influential theoretical framework that explains a large body of behavioral, electrophysiological, and brain lesion data in monkeys and humans (e.g., Chelazzi et al., 1993; Deco \& Zihl, 2004; De Weerd, Peralta, Desimone, \& Ungerleider, 1999; Gallant, Shoup, \& Mazer, 2000; Kastner, De Weerd, Desimone, \& Ungerleider, 1998; Moran \& Desimone, 1985; Reynolds et al., 1999; Treue \& Maunsell, 1999). With the term biased competition model we refer strictly to the empirically supported theory (Bichot et al., 2005; Reynolds et al., 1999), which has been mathematically implemented to include parallel competition across the visual field and a single biasing signal for target selection (Reynolds et al., 1999; see also Reynolds \& Heeger, 2009). The term competition refers to the finding that, in the absence of attention, the influences of two or more stimuli within a neuron's receptive field (RF) are averaged, thus providing little useful information for further processing of either stimulus at higher levels in the visual system (Moran \& Desimone, 1985; Reynolds et al., 1999; Treue \& Maunsell, 1999). RF size increases rapidly with higher hierarchical stages of visual processing, until they are almost as large as the entire visual field (Desimone \& Ungerleider, 1989). Therefore, for any set of stimuli in the visual field, it is true that their information will be mixed (and thus lost) at some level of the visual system. To overcome this problem, the competition is "biased" by attention toward a relevant stimulus (the target) so that for all the neurons containing the two stimuli in their RF, only the target's information is represented, at the cost of the irrelevant stimulus (the distractor). Paying attention to two stimuli during a divided attention task would have the effect only of giving equal weight to the stimuli during averaging, which means that at the hierarchical level of the visual system where RFs are large enough to encompass the stimuli, the information about the individual stimuli will be lost. Thus, full processing of two (or more) stimuli at the same time (i.e., divided attention) is not compatible with biased competition theory (see Discussion).

The weight of evidence and theoretical arguments that appear to support unified attention make clear that a potential finding of divided spatial attention would have far-reaching theoretical implications regarding the neural substrates involved in attentional control. On the basis of the discussed neurocognitive evidence, we suggest that participants in experiments will use a unified attention strategy whenever possible and that only in restricted conditions in which unified attention mechanisms might fail, there is the hypothetical possibility that they might divide spatial attention.
The term divided attention study may be misleading because it suggests that divided attention can be studied and thus presupposes the existence of such a phenomenon. Here, however, these studies are considered attempts to demonstrate spatial divided attention. In the following sections, we develop a formal approach to evaluate experiments investigating divided spatial attention.

\section{Divided Attention: Definition, Experimental Design, and Criteria}

To evaluate studies of spatially divided attention, we propose a testable definition of divided spatial attention, introduce commonly used experimental design features, and formulate four criteria against which evidence from these experiments will be evaluated.

\section{Definition of Divided Spatial Attention}

At first approximation, divided attention can be defined as the ability to carry out two or more attentional tasks in separate locations in parallel. A significant problem in the field of divided attention is that task performance predicted from parallel and serial models is often indistinguishable. A similar difficulty exists in the domain of visual search, where the determination of a theoretical model underlying search data is difficult because a data set is often compatible with particular implementations of both a parallel and a serial model (Townsend, 1990; Townsend \& Wenger, 2004). For example, the linear function between reaction time and the number of items to be searched observed in working memory (Sternberg, 1966) and visual search paradigms (Treisman \& Gelade, 1980) can be explained equally well by serial and parallel processing models (Atkinson, Holmgren, \& Juola, 1969; Egeth, 1966; Miller, 1988; Townsend, 1969). Likewise, in paradigms testing divided attention (divided attention paradigms), behavior that might be suggestive of parallel, simultaneous allocation of attention in two or several loci (split focus model) can easily be accommodated by serial models. The serial models that have been used to explain performance in divided attention tasks are of two types. According to a serial shifting theory of attention (B. A. Eriksen \& Eriksen, 1974; Posner, 1980), quasisimultaneous processing of two objects is accomplished by rapid shifting of a single, indivisible focus of attention that is fixed in size. According to a zoom lens theory of attention (C. W. Eriksen \& St. James, 1986), the attentional focus can be adjusted to match the spatial scale of the attended objects, and because processing within the focus of attention is assumed to take place in parallel, this could explain simultaneous processing of two target stimuli falling within the zoomed attentional focus. Thus, serial models could accommodate data suggestive of parallel processing in two ways. They might assume either rapid shifting of the attentional focus between separate stimulus locations (scanning) or attentional zooming so the focus of attention can encompass multiple information sources in the visual field. One might argue that both rapid shifting and zooming lead to attentional enhancement in between relevant locations and that this provides a way to distinguish serial from parallel allocation of attention; as in the case of parallel attention, there would be no attentional modulation in between relevant locations. However, the possibility exists that as the attentional focus moves from one to the next location, it is simply turned off (for a discussion, see Chastain, 
1992; Shulman, Remington, \& McLean, 1979; Tsal, 1983; Yantis, 1988; for reviews, see Cave \& Bichot, 1999; C. W. Eriksen \& Murphy, 1987). Thus, serial mechanisms are compatible with analogue shifts of attention in which attention remains always "on" and with discrete "hops" in attention in which attention is turned "off" during its movement. This renders it difficult to make differential predictions from serial and parallel models in divided attention tasks.

Because of the various ways in which a serial model could accommodate data from a paradigm testing divided attention, it is crucial to precisely define the concept of divided attention. We define divided attention as the ability to split attention into multiple, spatially noncontiguous distributions of enhanced processing that remain stable throughout the time the divided attention task or trial is carried out. The stability of a multimodal distribution of attention is essential: If attention moved from one target location to another during an individual trial, the attentional capacity available would not be truly split between locations. For the study of divided attention, it is irrelevant whether moving attention is accomplished through a serial mechanism (a shift or a hop) or a parallel mechanism during which attention would be turned up in one location while being turned down in the other. In both cases, the target stimuli could not be processed at once. The absence of a stable division of attention might be inherent to difficult tasks that require individual scrutiny of differently located stimuli. The absence of a stable division of attention can also be expected in easy tasks, in which the limited processing times in each location would permit quick movements of attention between target locations during stimulus presentation. Hence, the art of divided attention research consists of devising tasks that have the proper level of difficulty and the proper stimulus timing to enforce a true, temporally stable division of attention among relevant locations. The essential feature of the stability of such multifocal distribution leads to several methodological criteria, which, if satisfied, can help maximize the validity of a conclusion that a truly parallel division of attention underlies performance in a divided attention task.

\section{Main Experimental Design Features}

A first important design property in divided attention studies is the task participants are asked to perform. In one category of tasks, participants are instructed to detect, discriminate, or identify a single target that could be presented in two or more locations cued prior to target onset. The dependent measure is often the reaction time to the target (in case of a detection task) but can also be a measure of accuracy (in case of an identification or discrimination task). This task is referred to as a single-target experiment, with further specifications depending on the experiment. For example, if participants merely have to detect a dot in cued locations, we use the terminology single-target detection task. In the other task category, two or more targets have to be discriminated (i.e., identified, compared, or matched) simultaneously, and the dependent measure is usually the percentage of correct performance. This task is referred to as a two-target (or multiple-target) discrimination task.

A second design property is the manner in which attention is directed to relevant locations. Attention can be cued to one or more target locations with exogenous and endogenous cues. An exogenous cue is an attention-capturing stimulus presented away from a fixation point, which elicits an automatic orienting of covert attention to the cue (see Criterion 3: Appropriate cue-to-target intervals). An endogenous cue usually entails a symbolic instruction at fixation and a willful orientation to a particular location.

A third design feature of divided attention experiments is the manner in which the distribution of attention at and near target locations is measured (to verify whether attention is restricted to separate locations). In single-target detection tasks, the distribution of attention around target locations is tested by measuring detection performance for probing stimuli presented infrequently in nontarget locations. In (multiple) target discrimination or identification tasks, the distribution of attention is often tested by presenting distractors near the targets. In some experiments, the extent to which nearby distractors interfere with target discrimination or identification is used to measure attentional allocation away from the targets. In other experiments, the processing of the distractors themselves on occasional probing trials is used as a measure of the presence of attention away from the targets.

In the following section, theoretically motivated methodological criteria for good experimental design in divided attention studies are introduced. On the basis of an analysis of 19 prominent studies, we show that paradigms in which the criteria are not satisfied are not well suited to investigate the hypothesis of divided attention. Specifically, we show that failure to satisfy the proposed methodological criteria renders a unitary attention account of the data a plausible alternative to a divided attention account.

\section{Methodological Criteria}

Criterion 1: Sufficient task difficulty. To demonstrate divided spatial attention, a concentration of resources must be demonstrated at the target locations, and this is best achieved when stimulus processing requirements in the attention task approach attentional capacity limitations. According to the theorem of capacity limitations (Kahneman, 1973), only a limited amount of processing resources is available for the processing of stimuli or locations in the visual field, and reaching these limitations leads to a concentration of resources at the target location (Egeth, 1977; C. W. Eriksen \& St. James, 1986; Shaw \& Shaw, 1977). In line with that view, Lavie and colleagues (Forster \& Lavie, 2008; Lavie, 1995; Lavie \& Cox, 1997; Lavie \& Tsal, 1994) demonstrated that high stimulus processing load, also referred to as perceptual load (Yantis \& Johnston 1990; Miller, 1991), is a prerequisite for inducing a concentration of attentional resources. Their studies demonstrated that irrelevant information will be processed whenever target processing requirements fall within capacity limits but will fail to be processed when processing requirements reach or exceed capacity limits. On the basis of these findings, we suggest that in divided attention experiments, setting an appropriate task difficulty is instrumental for creating the conditions under which attention might be divided in separate distributions focused on the targets. Conversely, an easy task is unlikely to induce a concentration of attentional resources (and certainly does not require it) and is therefore unsuited to test divided attention.

Task difficulty can be calibrated by choosing stimulus parameters for which task performance in two locations leads to a performance decrement compared with performance in a single location (e.g., Braun \& Sagi, 1991). The smaller the detrimental 
effect of performing a task simultaneously in separate locations, the more doubts remain concerning the attention-demanding nature of the task, and the less likely it becomes that attention is split in separate foci that process the targets in parallel. Instead, when a task can be performed without cost in two locations, it is likely that the task can be performed in several more locations in parallel (if the task avoids working memory limitations).

Task difficulty is determined not only by the stimulus load but also by the type of operations that have to be carried out on the stimulus representations (e.g., detection vs. discrimination). It can be argued that discrimination experiments are generally more difficult than detection experiments, but both tasks could be made difficult by presenting stimuli at detection or discrimination threshold. Hence, task difficulty is determined by a mixture of pure perceptual load and of load related to cognitive operations carried out on the stimulus presentations. We use the term task difficulty with these qualifications in mind. Note that many factors can influence difficulty, such as the size, number, and density of display elements and the distances between targets and among targets and distractors (see, e.g., Miller, 1991). However, these factors have not been systematically investigated in divided attention studies.

In single-target detection tasks, participants often merely have to detect a small high-contrast dot. This task is trivial, and the only relevant dependent measure is reaction time. In single-target detection experiments, a sizable, significant increase in reaction time in multiple- versus single-location conditions might indicate that the task is attention-demanding nevertheless (at least several tens of milliseconds). Without that data, there is no reason to assume that attention is focused on the targets during the task (by either splitting, zooming, or serial reallocation).

In a two-target discrimination design, a sufficiently difficult task performed in a single location will result in good, but certainly not perfect, performance (e.g., 90\% correct). Simultaneous execution of the task in two (or more) locations should reveal a significant decrease from that performance level. We suggest that a decrease to a level in between chance and perfect performance or lower (e.g., $75 \%$ or less in each of two locations) would be clear evidence that the task is attention demanding and might require spatial focusing of attention.

In two-target discrimination tasks, the question whether the decrease in performance in the divided attention task is due to limitations in parallel or serial mechanisms can be tested by applying a behavioral paradigm proposed by Shiffrin and Gardner (1972). Once it has been demonstrated that carrying out a task in two (rather than one) locations entails a cost, performance in the divided attention task can be compared when two targets are simultaneously presented or sequentially presented. For example, participants might have to report the identity of two targets presented simultaneously for $50 \mathrm{~ms}$, or they might have to report the identity of one target presented for $50 \mathrm{~ms}$ followed by the other for $50 \mathrm{~ms}$ (in a predictable sequence). If low performance in the simultaneous condition were due to a serial mechanism under time pressure, presenting the targets in a predictable sequence should increase performance, because this would double the time during which the stimuli can be processed within the focus of attention. If low performance in the simultaneous condition were due to a parallel mechanism under time pressure, presenting the targets in a sequence would not help, as the processing time devoted to each target would remain unchanged. Note that the definition of divided attention as a temporally stable set of noncontiguous distributions provides the basis for the differential predictions in simultaneous and sequential conditions.

Finally, the number of training trials can be considered as an index of difficulty. It seems reasonable to consider studies in which participants perform the task at high performance levels after just a few tens of training trials as easier than a task in which thousands of trials are required before participants can perform the task.

Criterion 2: Sufficiently brief presentation times (control over stimulus processing). In both single-target and two-target experiments, brief target presentations may contribute to reaching an appropriate difficulty level and discourage serial strategies. Estimates of how short target presentations have to be to prevent voluntary attention shifts while still permitting target identification have been derived from data in a variety of paradigms. Studies using the attentional dwell paradigm (in which a stimulus is presented and masked in one location and followed by a stimulus and mask in a second location) have shown that the interstimulus interval required to identify both stimuli exceeds $200 \mathrm{~ms}$ (Duncan, Ward, \& Shapiro, 1994; Kröse \& Julesz, 1989; C. M. Moore, Egeth, Berglan, \& Luck, 1996; Peterson \& Juola, 2000; Reeves \& Sperling, 1986; Theeuwes, Godijn, \& Pratt, 2004; Ward, Duncan, \& Shapiro, 1996; Weichselgartner \& Sperling, 1987; for reviews, see Egeth \& Yantis, 1997; C. M. Moore et al. 1996). Studies using other paradigms suggest that much faster endogenous shifts of spatial attention are possible. In the divided attention task of C. W. Eriksen and Yeh (1985), participants had to move their attention in a subset of trials from a primary cued location where the target had not appeared to a cued or noncued secondary location in order to find the target. When the target was found in a noncued location, responses were delayed by about $110 \mathrm{~ms}$ compared with a response to a target in a primary cued location. When the most likely secondary target location was implicitly cued by the primary cue (i.e., participants knew where the target most likely would appear if not present in the primary location), responses were delayed only by $50 \mathrm{~ms}$. Kramer and Hahn (1995) desynchronized the onset of two stimuli that had to be matched (from 0 to $98 \mathrm{~ms}$ in 14-ms steps). The stimuli were presented briefly $(60 \mathrm{~ms})$, masked, and physical cues related to onset were attenuated (for details, see Kramer \& Hahn, 1995). Participants' attention was focused on the discrimination of targets at a single location, and only for a subset of target stimuli a shift of attention was required to assess the match with a target stimulus in a second location. For stimulus onset asynchronies (SOAs) exceeding 84 $\mathrm{ms}$, reaction times started to increase. Therefore, the longest onset asynchrony at which the increased reaction time was not observed yet (i.e., $70 \mathrm{~ms}$ ) is a reasonable estimate for the fastest possible serial shift in Kramer and Hahn's study. In difficult serial search tasks that require individual scrutiny of items in the search array, search times increased by roughly $100 \mathrm{~ms}$ per item (Bricolo, Gianesini, Fanini, Bundesen, \& Chelazzi, 2002). In another study in which participants were subjected to extensive training in a difficult search task, search performance suggested shifts of attention at a speed of $30 \mathrm{~ms}$ per item (Czerwinski, Lightfoot, \& Shiffrin, 1992). Electrophysiological studies in monkeys (Khayat, Spekreijse, \& Roelfsema, 2006; Motter, 1994a) and human participants (Chelazzi et al., 1993; Hillyard \& Anllo-Venlo, 1998; 
Mangun, 1995; M. M. Müller, Teder-Sälejärvi, \& Hillyard, 1998; Woodman \& Luck, 1999, 2003) in a variety of paradigms have demonstrated attention switches within 100-200 ms.

The evidence taken together suggests that depending on the paradigm used, shifts of attention could take place in a time window ranging from a few tens of milliseconds to $200 \mathrm{~ms}$ or more. On the basis of the evidence discussed, a reasonable criterion for studies of divided attention is to restrict stimulus exposures to $100 \mathrm{~ms}$ or less (taking into account task difficulty). Thus, if two target stimuli in a display are presented simultaneously, stimulus durations of $100 \mathrm{~ms}$ or less reduce the probability that a serial mechanism can account for their successful identification, on the condition that the task performed on the target stimuli is attention demanding.

There are three noteworthy issues regarding the requirement to minimize stimulus exposure duration. First, full control of processing time is afforded only by efficient masking (Averbach \& Coriell, 1961; Coltheart \& Arthur, 1972; Karni \& Sagi, 1991). Surprisingly, this is done in only 10 of the 19 studies that we have evaluated in detail in the present article. Without masking, an attention shift to a retinotopic location can be effective long after the stimulus disappeared because of still ongoing stimulus processing. Second, the exposure duration that must be set to prevent movements of attention will be shorter the easier the task is. Although an exposure duration of $100 \mathrm{~ms}$ may be sufficient to limit the probability of movements of attention in a difficult task (e.g., identification of complex target), this may not be the case in easier tasks (e.g., detection of a dot or identifying overtrained symbols such as letters), because limited processing requirements at each location may free enough time to move attention between locations (e.g., C. W. Eriksen \& Yeh, 1985; Hahn \& Kramer, 1995). Third, monitoring fixation is an important part of controlling stimulus exposure and retinotopic stimulus location. An important series of studies by Jordan and colleagues (Jordan \& Patching, 2006; Jordan, Patching, \& Milner, 1998, 2000, 2003) has shown that merely instructing participants to fixate a central point is not effective and that results from experiments obtained without fixation control are likely to produce misleading data. In the context of divided attention experiments, the absence of eye movement monitoring invites participants to foveate the stimuli or target locations by strategic, anticipatory saccades, especially when targets are shown in fixed locations and at predictable times in the trial. Moreover, participants may strategically adopt fixation positions away from the intended fixation point (e.g., closer to one of the target locations). The mix of attentional and eye movement strategies that participants may use in absence of eye movement control during divided attention tasks questions the validity of the data. Even when stimulus exposures are brief, and stimuli presented in unexpected locations, it is important to assess the variability of fixation behavior in different experimental conditions, as changes in variability will affect estimates of the spread of attentional distributions and associated conclusions about the potential division of attention. Specifically, although stimulus exposures of $100-150 \mathrm{~ms}$ render saccades during that time unlikely, there can be variability in the eye position relative to fixation at the time of stimulus onset. Remarkably, 11 of 19 divided attention studies we have analyzed did not monitor eye movements. Although a stringent evaluation of conditions that contribute to experimental control over the stimulus would lead to a disqualification of studies that did not monitor eye movements, we have assumed that the detrimental effect of not monitoring eye movements on its own does not completely invalidate the findings (especially for short stimulus durations). Hence, for the studies analyzed in this article, we merely mention the presence or lack of fixation control, without further discussion.

Criterion 3: Appropriate cue-to-target intervals. Spatial attention can be cued to a target location by endogenous and exogenous cues. Psychophysical experiments reveal different properties of exogenous and endogenous cuing that are relevant for the choice of the cue-to-target interval (e.g., Berger, Henik, \& Rafal, 2005; Fu, Caggiano, Greenwood, \& Parasuraman, 2005; Juola, Koshino, \& Warner, 1995; Steinman \& Steinman, 1998; Turatto et al., 2000). The effect of exogenous cuing is transient, and its attentional benefits are maximized in a 50-200-ms interval following the cue. Endogenous cuing can have sustained effects, and its benefits are maximized from $500 \mathrm{~ms}$ onward after the cue. In addition to differences in the time course of attentional allocation following exogenous and endogenous cuing, differences in the spatial distribution of attention have been reported. Studies using small stimuli as cues for spatial attention (Rizzolatti, Riggio, Dascola, \& Umiltà, 1987; Umiltà, Riggio, Dascola, \& Rizzolatti, 1991) or frames within which attention was to be directed (Downing \& Pinker, 1985; Hughes \& Zimba, 1985, 1987; Zimba \& Hughes, 1987) have reported a large spread of exogenous cuing effects, often encompassing an entire visual quadrant or hemifield. Using the line-spreading illusion, Steinman and Steinman (1998) reported more favorable estimates of the spread of exogenous cuing, but still extending up to $5^{\circ}$ surrounding the cue. The initially broad distribution of attention following exogenous cues may be refined by endogenous cues (instructions), which have been shown to be effective in setting up higher resolution attention distributions focused on the relevant cue (Egeth, 1977; LaBerge, 1983; Turatto et al., 2000; see also Busse, Katzner, \& Treue, 2008; Umiltà, Mucignat, Riggio, Barbieri, \& Rizzolatti, 1994). The endogenous zooming-in and precise delineation of attention may be facilitated by the use of a frame stimulus (e.g., Turatto et al., 2000) or by the presence of competing information in the visual field (for review, see Cave \& Bichot, 1999). Cortical processes may be predominant in the slower endogenous cuing effects (e.g., from the lateral intraparietal area, frontal eye fields, prefrontal cortex), and subcortical contributions may be predominant in the faster exogenous cuing effects (Berger et al., 2005; Boehnke \& Munoz, 2008; Buschman \& Miller, 2007; Corbetta \& Shulman, 2002; Juola et al., 1995; Sereno, 1992; J. J. Snyder \& Chatterjee, 2006). A strong subcortical contribution to exogenous cuing effects (Desimone et al., 1990; Wurtz \& Goldberg, 1972) is in line with the direct retinal input to superior colliculus and pulvinar, the large neuronal RFs in these structures (Gattass, Oswaldo-Cruz, \& Sousa, 1979; Goldberg \& Wurtz, 1972), and their roles in orienting (Sprague, 1996) and interhemispheric competition (Desimone et al., 1990; Peterson et al., 1987). The hypothesis of a fast orienting response (by exogenous cuing) followed by zooming in on a target (by endogenous cuing) is in line with Posner's view on attentional allocation stages (e.g., Posner \& Cohen, 1984).

Brief cue-to-target intervals can be important in easy singletarget detection experiments that use long or unlimited stimulus presentation durations and rely on speeded reaction times. With this type of design, a cue-to-target interval that is too long allows 
participants to select a single cued location prior to stimulus onset on a trial-by-trial basis and to shift attention to the other location if the target did not appear where attention was allocated initially, which can be done within $50 \mathrm{~ms}$ (C. W. Eriksen \& Yeh, 1985). Averaging data from such trials can lead to inferred attention distributions akin to spatial attention that has been divided (Posner et al., 1980). Specifically, if the time to shift attention is small compared with the magnitude and variability in reaction times, the effect of attention shifts on reaction times will be invisible in the average reaction time distributions to targets in the two target locations. Thus, a data pattern with fast reaction times in target locations, and slow reaction times in infrequently used probing locations away from target locations, may reflect a bias to allocate attention to target locations based on the expected probability of target presentation, rather than a parallel allocation of attention. In some studies (e.g., Shaw \& Shaw, 1977) this type of explanation seems applicable, and unless there are very aberrant features in the reaction time data (e.g., bimodal distributions), it cannot be excluded. Therefore, the results of single-target detection studies with long cue-to-target intervals are often difficult to interpret.

To avoid endogenous focusing of attention on a single location prior to target presentation, brief cue-to-target intervals as well as cue presentations at random times relative to trial onset are desirable features of single-target detection experiments. However, brief cue-to-target intervals can lead to a dominance of spatially unspecific exogenous cuing effects. Exogenous cuing following brief cue-to-target intervals, therefore, may prevent the focusing of the noncontiguous attentional distributions that are supposed to underlie divided attention. Hence, although a single-target detection (or discrimination) experiment may run into interpretational difficulties if the cue-to-target intervals are too long, it is not clear whether short intervals are a good alternative. On the basis of this reasoning, single-target detection experiments do not appear to provide an optimal test of divided attention.

If a single-target detection task were to be made difficult so that detection performance could be measured in terms of accuracy (as specified in Criterion 1), and exposure duration limited to prevent effective attention shifts, the problem of long cue-to-target intervals might be less severe. Under these conditions, better-thanchance performance on a single-target divided attention task with two target locations would argue against the idea that participants would have used the strategy of randomly selecting a single-target location prior to target onset (in which case performance would be $50 \%)$.

In two-target discrimination experiments that require a comparison or identification of both targets, the cue-target delay is best kept long to maximize the possibility that a divided attention distribution is set up according to instructed probabilities, and to overcome the initially broader distribution of attention induced by exogenous cues. As discussed for single-target experiments, a long cue-to-target interval is defensible only if stimulus exposure duration is brief and the task sufficiently difficult.

In summary, in easy single-target experiments, long cue-totarget intervals render the data uninterpretable. Hence, brief cueto-target intervals (and thus exogenous cuing) are required. By combining the benefits of a short interval to limit attention shifts $(<100 \mathrm{~ms})$ with the period in which exogenous cuing is maximized (50-200 ms), it seems that a cue-to-target interval of $150 \mathrm{~ms}$ or less might be a reasonable choice. However, in light of evidence for coarse attention distributions following exogenous cuing, the resulting attention distribution should be tested carefully to make a credible claim for divided attention. In difficult multiple-target experiments (or difficult single-target experiments) with short target exposures, cue-to-target intervals are best kept long (>500 ms) to maximize the possibility that noncontiguous attention distributions are set up at target locations. It is worth noting that in addition to the cuing stimuli, the target, distractor, and probing stimuli will be associated with a mixture of endogenous and exogenous cuing effects. Attentional allocation during target presentations is therefore a result of a complex interplay among the various attentional cues of the different stimuli presented during an experimental trial.

Criterion 4: A full reconstruction of the attentional landscape surrounding target locations. In divided attention tasks, it is important to demonstrate that attentional enhancement is limited to the target locations and absent in other locations in the visual field. This is accomplished by showing poor processing of probing stimuli or distractors presented away from the targets. If the results confirm that the processing of the probing or distractor stimuli is limited, it is inferred that attention must have been split.

The number and density of locations surrounding target locations that must be tested to establish that attention is directed to noncontiguous locations depends on the assumed flexibility of the attentional focus and on its spatial resolution. In many studies, limited flexibility is assumed, and stimulus placement is based on the idea that attention can be allocated only to round regions in the visual field, as suggested by the metaphor "focus of attention." If the assumption of limited flexibility is correct, the absence of attention at a single location in between two target locations would be sufficient to infer that spatial attention was divided.

In discussing the flexibility and resolution of spatial attention, we focus on endogenous attention, which offers the best chances for allocating attention to distinct locations at a spatial resolution that is high enough to potentially demonstrate divided attention (see Criterion 3: Appropriate cue-to-target intervals). Studies using sustained distributions of spatial attention induced by endogenous cuing have demonstrated that the attentional spotlight can be focused in highly flexible ways (Somers et al., 1999; for review, see Cave \& Bichot, 1999). For example, stimuli in the center of the spotlight can be ignored, indicating that attention can be deployed in an annular configuration (Juola, Bouwhuis, Cooper, \& Warner, 1991; M. M. Müller \& Hübner, 2002). Moreover, during reading attention is serially shifted from word to word, in agreement with a perceptual span of up to 15 characters in length and one character in width (Pollatsek, Reichle, \& Rayner, 2006; Reichle, Pollatsek, Fisher, \& Rayner, 1998; but see Engbert, Nuthmann, Richter, \& Kliegl, 2005). This finding suggests that the spotlight can take on a stretched (narrow) elliptical shape. Furthermore, monkey neurophysiological studies have shown that selective attention to one of two stimuli placed within the RFs of a visual neuron in early visual areas will lead to preferential processing of the attended stimulus by that neuron (Luck, Chelazzi, Hillyard, \& Desimone, 1997; Reynolds et al., 1999). The lowest level visual area where this finding has been reported is V2, and therefore V2 RF sizes (see, e.g., Gattass, Gross, \& Sandell, 1981) may be considered as an upper limit for the spatial resolution of spatial attention. Hence, the specificity of spatial attention can be estimated to be better than 
$1^{\circ}, 2^{\circ}$, and $4^{\circ}$ at eccentricities of $2.5^{\circ}, 5^{\circ}$, and $10^{\circ}$, respectively. These estimates of the resolution of spatial attention are in line with findings in human psychophysical studies (Intriligator \& Cavanagh, 2001; Sagi \& Julesz, 1986). Thus, contrary to assumptions in many studies of divided attention, a significant body of evidence indicates that spatial attention can be allocated in flexible, unified spatial conformations at high spatial resolution. ${ }^{1}$

In view of the flexibility and resolution of spatial attention, divided attention studies that use sparse stimulus arrangements cannot exclude the possibility that cued target locations are encompassed by a unified distribution of attention shaped to avoid irrelevant areas or influences of distracting stimuli. Specifically, in many studies a limited number of target and probing or distractor stimuli are arranged equieccentrically around a fixation spot (usually done to equate visibility). As a consequence, a narrow elliptical focus could incorporate the two relevant locations without incorporating the distractors or probes in so-called midlocations. In other studies, the stimuli are arranged on a straight line, often with a target location to the left and to the right of a fixation point, with probing stimuli or distractors falling in between. This is another stimulus arrangement in which lack of processing of probing stimuli or distractors does not imply divided spatial attention, because a slightly bent elliptical or annular focus, or an annular region of attentional enhancement, could be set up to include targets while avoiding the probing or distractor locations.

To reject the hypothesis that the focus of attention adopts a single contiguous region of enhancement that is shaped to optimize performance, the experimental design of divided attention studies should include probing stimuli (distractors) in many unpredictable locations in between and surrounding the target locations. The spacing among stimuli and probing locations should minimize the possibility that a unitary distribution of attention could be formed that avoids distractor-probing locations while still encompassing target locations. We suggest the use of empirically determined estimates of the spatial resolution of endogenous attention as a guideline to choose sufficiently dense stimulus or probing locations at different eccentricities (based on psychophysics and neurophysiology). If probing stimuli or distractors are presented at a sufficient spatial density across the display, a plausible reconstruction is possible of any landscape of attentional allocation surrounding the targets. This permits drawing unbiased conclusions, because a conclusion of divided attention will be based on evidence that the unified attention is not true, or vice versa. Hence, any study that claims spatial divided attention without having tested the unified attention hypothesis cannot come to a credible conclusion of divided attention.

\section{Using Strong Rather Than Weak Criteria to Assess Plausibility}

The criteria we use to evaluate studies of divided attention can be summarized as follows: We suggest that a sensible investigation into split spatial attention requires a demanding stimulus and/or task (Criterion 1), short stimulus exposures $(<100 \mathrm{~ms})$ and masking to prevent shifts of attention during target presentation (Criterion 2), a cue and a cue-to-target interval that help to establish a stable attention distribution prior to target presentation (Criterion 3), and a demonstration of the absence of attentional enhancement surrounding the targets by a sufficiently complete assessment of the attentional distribution surrounding target locations (Criterion 4).

It could be argued that it would be fairer to test studies of divided attention against more relaxed criteria and still to accept claims of divided attention from studies with somewhat flawed designs, in light of the cumulative nature of scientific progress. However, the credibility of a conclusion of divided attention in specific experimental conditions hinges on a successful rejection of the unified attention model in these conditions (Popper, 1959), and we doubt that cumulated evidence from experiments that all have an identifiable design flaw could make a much stronger case against unified attention than any of the flawed experiments themselves. In our view, the accumulation of data collected with imperfect experimental designs does not carry enough weight to firmly reject the null hypothesis that spatial attention cannot be split. $^{2}$ To the contrary, a phenomenon that appears to be reported only under less than rigorously controlled conditions should be put in doubt. We argue that a rejection of the null hypothesis that spatial attention is indivisible cannot be based on studies that leave open an alternative scenario for the null hypothesis to remain true, especially when this alternative is plausible, empirically supported, and could be excluded by better experimental design. Hence, the outlined criteria are useful, as a study that fulfills them would provide a strong argument against a unitary focus of attention, and fair, as it is entirely possible to design a task that fulfills them.

To avoid ambiguities in the interpretation of the different criteria, it is worth highlighting that we use the four criteria to evaluate the plausibility of a conclusion of divided attention, without denying the logical possibility of divided attention when criteria are not satisfied. For example, it is logically possible that in a psychophysical study failing Criterion 1 (easy task) a convincing finding of divided attention would be reported. However, on the basis of the empirical studies underlying Criterion 1, we consider this unlikely. Likewise, even in the extreme case in which the experimental task is too easy (failing Criterion 1), stimulus exposures and cue-to-target intervals are too long (failing Criteria 2 and 3 ), and the density of probing-distractor stimuli is too low (failing Criterion 4), the logical possibility that participants used divided spatial attention cannot be excluded. However, under these nonrestrictive conditions, many attentional strategies are possible (unified attentional strategies being most likely), making a conclusion of divided attention untenable. In general, we suggest that the more convincingly all criteria are fulfilled, the more plausible a potential conclusion of spatial divided attention can be.

Note that a demonstration of a stable division of attention in unconstrained stimulus conditions (i.e., not in accordance to the four criteria) requires methods that can measure relevant data at high spatial and temporal resolution. This is most easily achieved through invasive recordings of neuronal population activity in animals. For example, using a dense array of electrodes targeting central vision in monkey V4, a divided attention task may reveal enhanced spontaneous activity and synchrony in separate popula-

\footnotetext{
${ }^{1}$ If attention can be directed to regions of attention with a flexible shape, it might be useful to reinterpret the metaphor of a "focus" of attention as a region in the visual field where attention brings stimuli "in focus," instead of a region of enhanced processing that has the shape of a "focus."

${ }^{2}$ After an anonymous reviewer.
} 
tion of neurons with nonoverlapping RFs in locations where the target stimuli are expected (Engel, Fries, \& Singer, 2001) but not in other, surrounding locations. This pattern of neuronal activity can be established in periods without any stimulation, and could be confirmed by measuring responses to probing stimuli occasionally presented during performance of the divided attention task. It may be possible to use these direct, high-resolution measures of brain activity to establish a sustained, stable division of attentional resources lasting for many seconds, and in this context a number of stimulus restrictions proposed in Criteria 1-4 may be less relevant. However, equivalent direct approaches in humans are ordinarily not available. Given the spatial (magnetoencephalography, electroencephalography) and temporal resolution (fMRI) limitations of indirect methods to measure human brain activity noninvasively, and the indirectness of purely psychophysical approaches, conclusions of divided attention risk lacking plausibility on methodological grounds alone if stimulus conditions have not been chosen to exclude as much as possible a unified attention strategy (i.e., zooming or shifting attention, or deforming its distribution to avoid distractor locations). Hence, in addition to the theoretical reasons that make a division of spatial attention unlikely in unconstrained conditions, there are methodological reasons that suggest it would be very difficult to demonstrate divided attention in human experiments using unconstrained experimental conditions.

\section{Other Considerations: Dividing Attention Among Object Features, Among Objects, and the Role of Suppression}

The question how attention is divided can be asked not only in the domain of spatial attention but also in the domain of featurerelated and object attention. In the domain of feature-related attention, some studies indicate a competition for attentional resources when tasks are limited within the same feature domain, but no competition across feature domains. For example, while performing a contrast increment detection task in the luminance domain in one location, performing a second task in another location decreases performance, but only if the second task is also in the luminance domain, and not if the second task is in the color domain (Chirimuuta, Burr, \& Morrone, 2007; Morrone, Dente, \& Spinelli, 2002, 2004). We speculate that the effects of competition between features would probably be present in locations other than the tested target locations, as enhancement produced by attention to single features also leads to effects across the visual field (Bichot et al., 2005). In support of the parallel nature of attentional feature processing and competition between features, Chirimuuta et al. (2007) showed that after training in specific locations on the simultaneous performance of tasks in two feature domains, the acquired skill generalized across the visual field. Thus, the experiments by Morrone et al. $(2002,2004)$ and Chirimuuta et al. can be seen as evidence for a parallel competition between feature processing across the visual field and capacity limitations in the parallel processing of features. This is different from the study of the effects of capacity limitations during task performance in two spatial locations, which is the subject of the present article.

In addition to directing spatial attention and attention to object features, attention can be directed to complex objects as a whole. Object-based attention can operate independently from spatial attention, as it can be selectively directed to one of two objects superimposed on the same location (O'Craven, Downing, \& Kanwisher, 1999). Furthermore, object-based attention differs from feature-based attention, as one can attend a specific feature of an object while ignoring other features (Nobre, Rao, \& Chelazzi, 2006). The manner in which these different forms of attention interact is debated. Several studies have demonstrated that the division of attention between object features is facilitated when the features belong to the same rather than different objects (e.g., Driver \& Baylis, 1989; Duncan, 1984; Egly, Driver, \& Rafal, 1994; Mattingley, Davis, \& Driver, 1997). More recent evidence suggests that the inefficiency of attending to parts of two objects may be related to the spreading of spatial attention across the spatial extent occupied by the objects, rather than to a fixed inability to process more than one object at a time (Davis, Driver, Pavani, \& Shepherd, 2000). The division of object-related attention, and the ways in which different forms of (divided) attention interact, go beyond the scope of the present article.

Attention distributions are often understood to reflect variations in processing enhancement, but in stimulus arrays containing distractors, enhanced processing at the target location may be accompanied by suppressed processing of surrounding distractor stimuli. Suppression outside the attended locus has been demonstrated by psychophysical as well as neuroimaging studies (Bahcall \& Kowler, 1999; Caputo \& Guerra, 1998; Cave \& Zimmerman, 1997; Cepeda, Cave, Bichot, \& Kim, 1998; M. M. Müller \& Hübner, 2002; N. G. Müller \& Kleinschmidt, 2004; N. G. Müller, Mollenhauer, Rösler, \& Kleinschmidt, 2005; Slotnick, Hopfinger, Klein, \& Sutter, 2002; Slotnick, Schwarzbach, \& Yantis, 2003; Smith, Singh, \& Greenlee, 2000), and may be related to negative blood oxygen level dependent (BOLD) signal (Shmuel et al., 2002; Smith et al. 2000; Smith, Williams, \& Singh, 2004). If performance in a divided attention task would reveal that the regions of enhanced processing at the target locations are surrounded by an area of suppressed distractor processing, and these suppressed areas join, then it can be asked whether this supports divided spatial attention. The answer depends on whether the notion of an attentional focus is expanded to include suppressive regions, which would lead to a redefinition of the focus of attention as an area of attentional modulation. According to that definition, separated regions of attentional enhancement embedded in a larger region of suppression belong to the same attentional focus. Hence, suppressive processes add a level of complexity to testing the concept of divided spatial attention. However, most studies do not address the topic of suppression, and we consider attention divided if there is evidence for two or more noncontiguous regions in the visual field where attentional performance exceeds that in the rest of the visual field.

In the classical attention literature (e.g., Treisman, 1982; Treisman \& Gelade, 1980), attentive is equated with serial processing, and preattentive is often equated with parallel processing. There is now evidence that also parallel processes may require attention (Joseph et al., 1997), and even that some serial processes may take place preattentively (VanRullen, Reddy, \& Koch, 2004). This suggests that the attentive-preattentive dichotomy is not identical with the serial-parallel dichotomy. In the context of our analysis of divided attention studies, parallel and serial processes are both attentional strategies that could be employed by a participant 
instructed to perform a task simultaneously in more than one location.

Psychophysical studies of divided attention (e.g., Egly \& Homa, 1984; H. J. Müller \& Findlay, 1987; Shaw \& Shaw, 1977) have often provided the inspiration for the paradigms and stimuli used in neurocognitive studies. Psychophysical studies therefore are discussed first, followed by electrophysiological and imaging studies. Much of the divided attention literature is difficult to read because of the different terminology, stimuli, and paradigms used in different studies, and other differences among studies. We chose to highlight details of stimuli and paradigms while using consistent terminology across studies to permit the reader to arrive at an informed evaluation of the data without having to refer to the original studies. The analysis below will highlight the main design criteria on which a study fails, with the outcome of all criterion evaluations for each study summarized in Table 1 (see Discussion).

\section{Psychophysical Studies}

Psychophysical studies of spatial divided attention can be subdivided into three categories. First, we discuss single-target detection and identification experiments. The task in these experiments is either to detect a single dot, or small disks, or to identify a specific target letter. Typically, the distribution of attention is tested with probing stimuli, which are target stimuli presented in nontarget locations in a minority of trials. Performance is ordinarily analyzed in terms of reaction times. Second, we discuss multiple-target identification or matching experiments. The task in these experiments is to identify or match letters or shapes presented in multiple locations (usually two). As in single-target experiments, the distribution of attention is usually tested by probing stimuli. Some studies also test the distribution of attention near target locations by measuring the interference of nearby distractors on target performance (distractor interference). Third, we consider as a separate category multiple-target experiments in which participants are monitoring locations in which rapid serial visual presentation (RSVP) streams are shown or are tracking dynamically moving targets. The stimuli used typically are letters and digits in RSVP streams and simple dots or shapes in tracking tasks.

\section{Single-Target Detection and Identification Experiments}

Single-target identification in two target locations after exogenous cuing. C. W. Eriksen and Yeh (1985) devised an experiment in which participants monitored information presented simultaneously in several relevant locations, under conditions that would favor either serial or divided allocation of attention. In their main experiment (see Figure 1), eight small stimuli were displayed together at a given eccentricity $\left(2.5^{\circ}\right.$ in their "large" displays) around a fixation spot. Participants were instructed to identify a target letter $(S$ or $Y$ ), which could appear in four possible locations (at 3, 6, 9, or 12 o'clock). Stimuli in the other locations were distractors (see Figures 1A and 1B). They had to be ignored and were not used to test attention away from target locations. In the experimental condition, $150 \mathrm{~ms}$ before display onset, a single bar-shaped cuing stimulus was presented near one of the four possible target locations. This cue indicated that a target was likely to occur not only in that location (the primary location) but also in the diametrically opposite location (secondary location). The probability that a stimulus would occur in the primary, the secondary, or the two noncued target locations was varied in three experimental conditions. In the first condition (40-40-10-10), the target appeared on $40 \%$ of the trials in the primary location, on $40 \%$ of the trials in the secondary location, and on $10 \%$ of the trials in the two remaining (noncued) target locations. In the second condition (70-10-10-10), the target appeared on $70 \%$ of the trials in the primary location and on $10 \%$ of the trials in each of the remaining three locations. In the third condition $(100-0-0-0)$, the target always appeared in the primary location. Participants were informed of these probabilities prior to each condition. Stimuli were presented tachistoscopically, but eye movements were not monitored. Average reaction times varied in a 420-560-ms range in the different conditions. Accuracy was not reported but can be assumed to have been close to perfect.

C. W. Eriksen and Yeh (1985) hypothesized that attention would be allocated according to the probabilities of target presentation in the target locations. They predicted that the first condition (40-40-10-10) would induce a division of attention, with equal reaction times expected for both cued target positions. A serial strategy was anticipated in the 70-10-10-10 condition, in which participants were strongly biased to expect the target in the primary target location. Here shorter reaction times were expected for the primary location than for the secondary and noncued locations. In contrast to these predictions, the data were in agreement with the serial alternative in both experimental conditions. Reaction times to letters in the primary target location were always shorter than reaction times to letters in secondary or noncued target locations, with the longest reaction times found in noncued locations. In the 40-40-10-10 condition, responses to secondary or noncued locations were delayed by about 50 and $110 \mathrm{~ms}$, respectively, compared with the primary location. In the 70-10-10-10 condition, responses to secondary or noncued locations were delayed by about 80 and $120 \mathrm{~ms}$, respectively, compared with the primary location.

C. W. Eriksen and Yeh (1985) suggested that participants had a preset serial strategy to attend the primary location first and to attend the secondary location or noncued location later, even in the 40-40-10-10 condition where a divided attention strategy seemed more likely a priori. Although the interpretation of the global pattern of data in terms of a shift of attention is reasonable, the interpretation of the data in the 40-40-10-10 condition as evidence in favor of a preset serial strategy is not. The investigators cued the primary location directly with a small bar stimulus preceding the target stimulus and cued the secondary location only indirectly (by its fixed spatial relationship with the location of the primary cue). The time between the cue and the stimulus onset was $150 \mathrm{~ms}$, which makes the cued location subject to exogenous facilitation, at the disadvantage of the secondary location (see Criterion 3). Thus, also in the 40-40-10-10 condition where target probabilities were equal in primary and secondary location, the primary location was cued more effectively than the secondary location. This imbalance in the strength of cuing might have contributed to a strategy of shifting attention. In addition, the probing locations in between and surrounding the cued target locations that would have been required to test a hypothesis of 
A

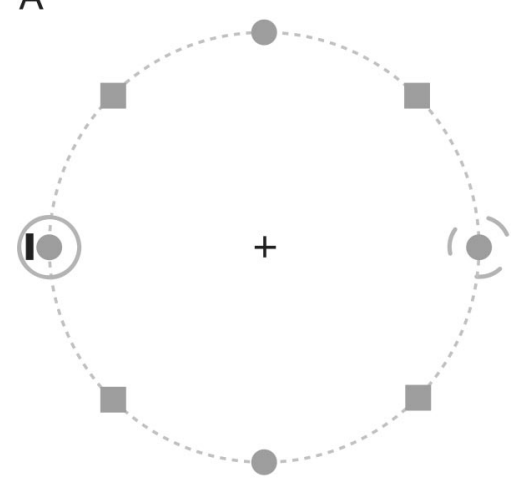

Direct cue

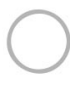

Directly cued location

( Indirectly cued location

-

Possible target location

Distractor location

Imaginary circle constraining stimulus layout

B

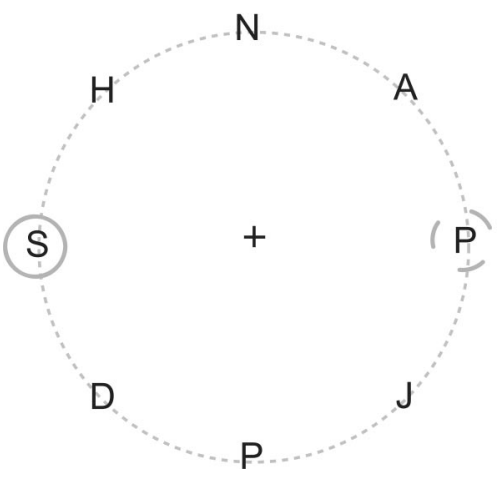

C

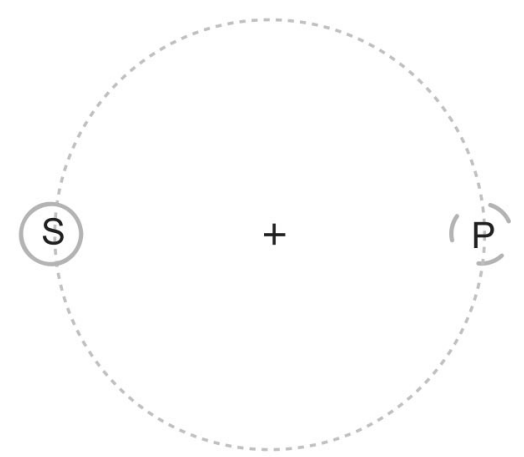

Figure 1. Overview of stimulus displays (not to scale) in the experiment of C. W. Eriksen and Yeh (1985). Gray circles, dots, and squares are for illustrative purposes and were not present in the stimulus displays. In "large displays" eccentricity of the stimuli was $2.5^{\circ}$; in "small displays" eccentricity was $1^{\circ}$. Results from both display sizes were pooled, as they were indistinguishable. The width and height of the characters were $0.16^{\circ}$ and $0.20^{\circ}$, respectively, and a similarly sized bar $\left(0.20^{\circ} \times 0.08^{\circ}\right)$ was used as a cue. (A) A maximum of eight stimuli as indicated by gray dots and squares was presented in a given display, equieccentrically around fixation (dashed circle). Targets were $S$ and $Y$ symbols. There were four predefined potential target locations (illustrated by small dots) and four distractor locations (small squares). In any display, there were two cued target locations. One location was cued directly by a small bar stimulus (small solid circle outline), the other indirectly by virtue of being opposite to the primary cue (small dashed circle outline). The two noncued target locations where targets are shown at a low probability can be considered probe locations. (B) Example of a display with eight stimuli, with a target $(S)$ in the directly cued location and a distractor $(P)$ in the indirectly cued location. (C) Example of a similar display with stimuli only in the target locations.

divided attention were lacking (Criterion 4). Furthermore, the task was easy, and the stimuli were presented unmasked (Criteria 1 and 2 ), and therefore serial shifts cannot be fully excluded in any of the conditions of this experiment. Hence, the experimental design used by C. W. Eriksen and Yeh precluded a solid conclusion regarding the presence or absence of parallel, divided attention.

Single-target detection in two target locations cued with differently sized frames (endogenous cuing). Castiello and Umiltà (1992) performed a series of experiments aimed at reinvestigating the possibility of parallel processing at two locations (eye fixation was monitored). The participants' task was to respond as fast as possible to the onset of a small dot. To cue the relevant locations within which the dot could appear, two empty frames (outlines of squares) were presented together $10^{\circ}$ to the left and right of fixation. The sizes of the two frames were never the same but varied on different trials (see Figure 2A). The target dot
(100 ms) appeared at random in one of the two frames. Cue-totarget intervals of $500 \mathrm{~ms}, 600 \mathrm{~ms}$, and $50 \mathrm{~ms}$ were used, and trials with these different intervals were randomly interleaved. The frames stayed on until the end of the trial. Average reaction time in the experiments with the long cue-to-target intervals was on the order of $260 \mathrm{~ms}$, with slightly longer reaction times $(337 \mathrm{~ms}$ ) for the 50-ms cue-to-target interval, and performance correct was near $100 \%$.

The data for the 500-ms cue-to-target interval showed that reaction times were smaller for dots presented in the small frame of $1.1^{\circ}$ (226 $\mathrm{ms}$ in their Experiment 1) compared with dots presented in the largest frame of $3.3^{\circ}$ (264 $\mathrm{ms}$ in their Experiment 1). The results were replicated with frames that were merely indicated by dots on the four corner positions, showing that the results were not contaminated by contextual interactions. The effect of frame size on reaction time was not 
A

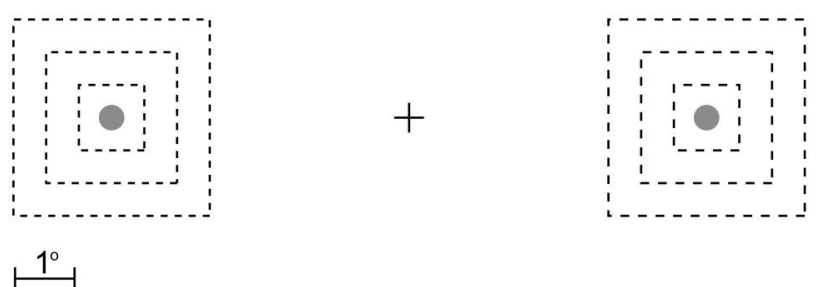

B

\section{Double-frame condition}

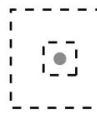

-

Single-frame conditions

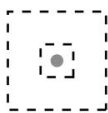$$
+
$$

$\bullet$

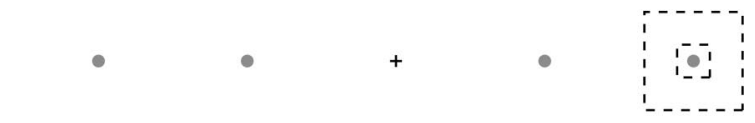

$1_{1}^{\circ}$

- Possible target location

. - . - Outline of possible cuing frames

Figure 2. Overview of conditions in two experiments with frames as attentional cues (drawings to scale). (A) Design and stimuli used by Castiello and Umiltà (1992). A single target could appear in one of two locations $5^{\circ}$ to the left or right of fixation (gray dots), both of which were cued simultaneously by frames (dashed lines) sized $1.1^{\circ}, 2.2^{\circ}$, or $3.3^{\circ}$. All sizes are illustrated, but actual trials presented only a single frame per position. (B) Design and stimuli used by McCormick et al. (1998). A single target could appear in one of four locations $5^{\circ}$ or $10^{\circ}$ to the left or right of fixation (gray dots). Two frame sizes were used $\left(1.1^{\circ}\right.$ and $\left.3.3^{\circ}\right)$ in a double-frame condition but also in a single-frame condition. In the singleframe conditons, targets could be validly or invalidly cued by the preceding frames.

present for cue-to-target intervals of $50 \mathrm{~ms}$. This suggests that a 500-ms cue-to-target interval permitted the focusing of attention on the outlined relevant location according to their sizes and that the exogenous cuing effects with the 50-ms cue-totarget interval were less spatially specific. The decrease in reaction time with decreasing frame size for the longer cue-totarget interval is in agreement with a parallel model in which a limited amount of attentional resources (C. W. Eriksen \& St. James, 1986) is split equally between the two locations, leading to less attention per unit of surface in the larger frame.
However, there are at least four factors that limit the interpretation of the data. First, because the task was very easy and the stimuli were not masked, serial shifts of a small focus of attention within and between attended regions cannot be excluded. The results are compatible with serial scanning of each outlined area, which would take less time for a small compared with a large attended area (Criteria 1 and 2). Second, a single focus of attention might have encompassed the relevant regions. A combined analysis of Castiello and Umiltà's (1992) experiments by McCormick et al. (1998) supported this possibility (see McCormick et al., 1998, Table 1). This analysis revealed that average reaction times increased with the area the attentional zoom would have to cover to include the two relevant locations (from $252 \mathrm{~ms}$ for the smallest zoom to $265 \mathrm{~ms}$ for the largest zoom). Because, on average, a larger frame will necessarily be associated with a larger attentional zoom and a smaller frame with a smaller zoom, this may at least in part explain the differences in reaction times between targets in small compared with larger frames reported in Castiello and Umiltà. Third, during the 500-ms cue-to-target interval, it is also possible that participants allocated a fixed amount of resources to either the left- or the right-hand location on a trial-by-trial basis, which would be compatible with the finding of slower processing in larger frames (Criterion 3). The assumption underlying this reasoning is that the time required for reorienting attention on trials where the a priori allocation of attention was incorrect is not so long and variable that it obscured the effect of increased reaction times for larger cued regions. Fourth, there was no test of the distribution of attentional resources away from target locations (Criterion 4). On the basis of these considerations, the data support neither unified models of attention (serial or zooming models) nor parallel, divided attention models.

Castiello and Umiltà's (1992) study was replicated by McCormick et al. (1998), who indicated that a split of attention is unlikely, and instead hypothesized that Castiello and Umiltà's data were produced by an enlarged focus of attention. To test the zooming hypothesis, they measured attentional modulation in between relevant locations. Eye fixation was monitored. They cued one or two locations designated by square frames $\left(1.1^{\circ}\right.$ or $3.3^{\circ}$ in size $)$ presented $10^{\circ}$ to the left or to the right of fixation (see Figure $2 \mathrm{~B}$ ). Participants reported the presence of dot stimuli in or near the cued regions. The target dot was presented $515 \mathrm{~ms}$ after onset of the frame, and both the frame and the target dot remained on the screen until the participant responded. There were three types of trials: with a single frame on the left of fixation, with a single frame on the right (single-frame conditions), or with a frame on either side of fixation (double-frame condition). In all three types of trials, a target dot could be presented in four possible locations (falling on a horizontal line through the fixation target). In the single-frame conditions, the dot could appear within the frame, in between the frame and the fixation spot, and in the two symmetrical locations on the other side of the fixation spot. In the doubleframe condition, two of the target locations fell within the frames, and the two others fell between the fixation spot and the frames. In any single-frame trial, there was a .615 probability that the target was presented inside the frame, with a much smaller probability (.077) for each of the remaining three locations outside the frame. In any double-frame trial, there was a .75 probability that a target appeared within one or the other frame (.375 for each frame), with a much smaller probability for the remaining two locations outside 
the frames (.0625 for each frame). The probability of a catch trial (without target) was about .15. Participants were aware of these contingencies.

The different cuing conditions were designed to test whether increasing the number of primarily attended locations (cued by frames) from one to two would lead to an increase in size of the attention focus to encompass both (zooming model) or would lead to two attention foci (splitting model). Both models predict that in the single-frame condition, reaction times to targets within the frame would be shorter than to targets outside the frame. However, in the double-frame condition, the two models make different predictions. According to the zooming model, reaction times to targets shown in between the two frames should be similar to reaction times to targets within a frame (because in-between targets fall within the enlarged attention focus). According to the splitting model, reaction times to targets shown in between the two frames should be larger than reaction times to targets within a frame (because in-between targets would fall in between the separate foci of attention). The main result of the experiment was that reaction times to targets inside and in between frames were similar in the divided attention condition. This indicates that attentional allocation in between frames was similar to allocation within frames.

Although the results seem to speak for a zooming model and against divided attention, several aspects of the experimental design precluded a conclusive interpretation of the data. First, the increase in reaction time when targets had to be detected in two locations rather than one was only $19 \mathrm{~ms}$, raising doubts whether the task was difficult enough to permit a plausible test of divided attention (Criterion 1). Moreover, it is possible that the increased reaction times in the dual-task condition were due to the low .375 probability of target presentation in each location compared with .615 in a single location (Criterion 3). A reduction of the probability of target presentation at a single location from .615 to .375 may have produced a similar increase in reaction time. Second, the target and frame remained on the screen until the participant responded, leading to presentation times that were too long to exclude reallocations of attention during stimulus presentation (Criterion 2). With reaction times in the order of 250-400 ms, the focus of attention could have shifted between the two frame locations, thereby leading to the attentional modulation of locations in between target locations. Third, because of the long cue-to-target intervals, it is unclear to what extent allocation of attention to single frames prior to target onset has contributed to the results in the dual-frame conditions (Criterion 3). With respect to cuing, the effectiveness of target probabilities in target locations to spatially direct attention can be questioned. It is open to doubt that attention would be centered exclusively at the two frame locations, each at the opposite side of the fixation spot at $10^{\circ}$ of eccentricity, because in only $37.5 \%$ of trials a target was presented at each location and because the monitoring of in-between locations is easier (because closer to fixation). The ineffectiveness of the peripheral cues in the present experiment to effectively "collect" attention is also indicated by the very small difference in reaction time between small $\left(1.1^{\circ}\right)$ and large $\left(3.3^{\circ}\right)$ frame conditions $(6 \mathrm{~ms})$. Fourth, note that the large stimulus spacing and lack of probing locations surrounding target locations precluded a complete assessment of the distribution of attention in the stimulus displays used (Criterion 4). In conclusion, the chosen design parameters make the data from this study hard to interpret.

Single-target detection in two locations cued with masked cues. It has been shown that attention improves spatial resolution, thereby leading to improved accuracy of perception, increased discrimination performance, and shorter reaction times (Deco, Pollatos, \& Zihl, 2002; Frey, 1990; Intriligator \& Cavanagh, 2001; Schneider \& Bavelier, 2003; Shore, Spence, \& Klein, 2001). In addition, attention has been demonstrated to speed up processing. Of two simultaneously presented stimuli, the onset of the attended stimulus is perceived earlier than the nonattended one, a phenomenon termed prior entry (Titchener, 1908). Prior entry can be induced by endogenous or exogenous cues. Exogenous cues can speed up processing if they are presented in sufficiently close spatial and temporal proximity of the target stimulus. When their spatial and temporal proximity is very close, the exogenous cue can become masked by the target (Breitmeyer \& Kersey, 1981; Enns \& Di Lollo, 2000). It is interesting that the attentional enhancement or prior entry of the target stimulus (Jaskowski, van der Lubbe, Schlotterbeck, \& Verleger, 2002; McCormick, 1997; Scharlau \& Neumann, 2003a, 2003b) can take place despite a strong reduction or even an absence in awareness of the cue itself due to masking (Klotz \& Neumann, 1999; Scharlau \& Ansorge, 2003). This masked-cuing paradigm was used by Scharlau (2004) to investigate the division of attention between two noncontiguous locations in the visual field.

Scharlau (2004) aimed to contrast models that assume a unitary focus of attention (shifting model and zooming model) with a model in which attention can be split into multiple foci in noncontiguous regions of the visual field (splitting model). In their experiments, participants were asked to judge the order in which two differently colored disk outlines were presented (e.g., "red before green" or "green before red"). These target stimuli were effectively presented with different onsets, the standard target (e.g., green) being presented at a fixed time in the course of the trial and the variable target (e.g., red) being presented at a variable time interval before (negative SOA) or after the standard (positive SOA). The variable stimulus was always presented simultaneously with a distractor stimulus (in a third color; e.g., yellow). The stimuli were presented in one of two possible triangular formations centered on the fixation spot (one possible arrangement is shown in Figure 3). Colors of distractor and targets were fixed in each participant, and targets and distractor positions in the triangular formation were varied in position from trial to trial, in a balanced way, so that participants had to rely exclusively on the color of the targets to select the stimuli for which a judgment of temporal order was required. Fixation was not monitored. Disk outlines had a diameter of $1.9^{\circ}$ and an eccentricity of $5.6^{\circ}$. The cue consisted of a disk outline perfectly fitting inside the cued target or distractor, with the same color as the cued stimulus. The cue $(16 \mathrm{~ms})$ was followed immediately by the cued stimulus, which stayed in view until the participant gave a response. Note that we classify the present study as a single-target detection design because participants detect a single target preceding another one in time.

In the first experiment (see Figure 3), four conditions were used, with the last one devised to falsify the unitary focus theory. First, in an uncued condition, the two targets were presented with different SOAs ( -48 to $48 \mathrm{~ms}$ ) without attentional manipulation. In this condition, the psychometric curve plotting the proportion of responses "standard before variable" against SOA showed $50 \%$ 


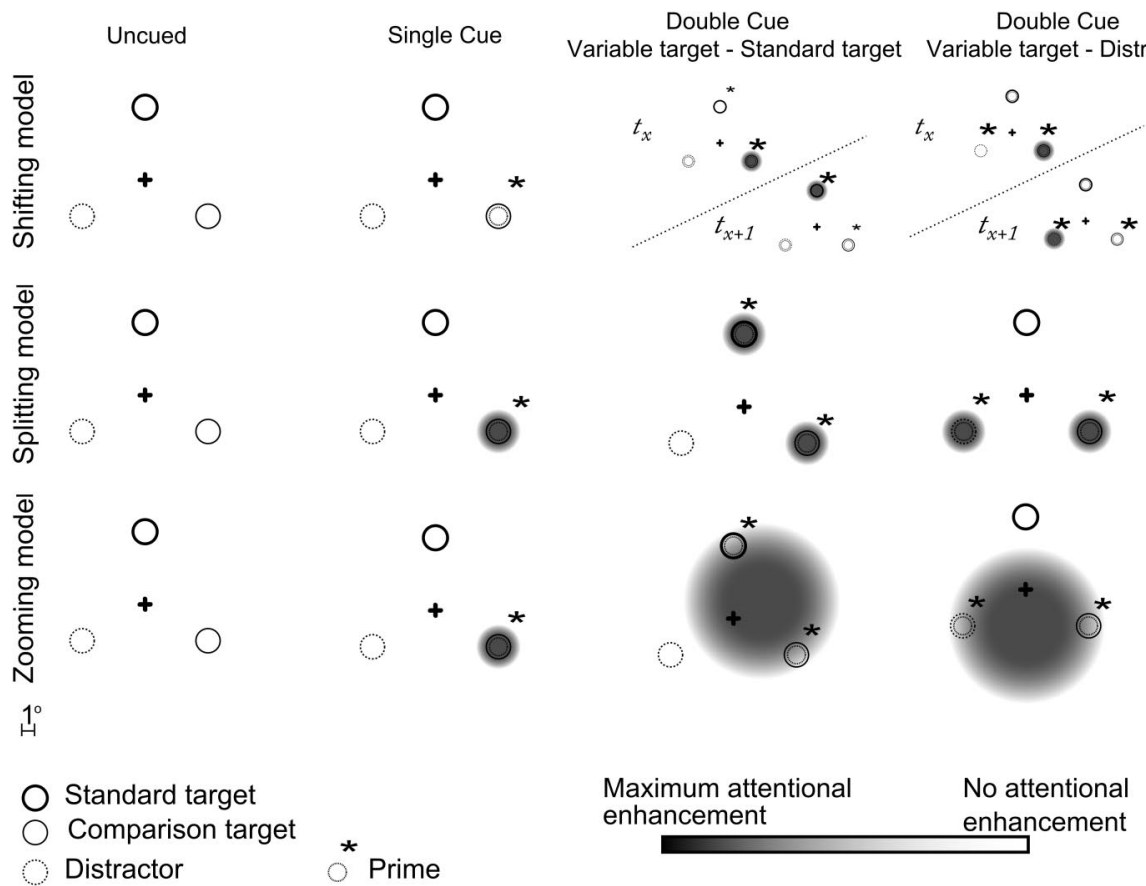

Figure 3. Experimental conditions in the prior-entry experiment of Scharlau (2004). Hypothesized shapes of attention distributions (radial grayscale gradients) are shown in four cuing conditions (four columns) according to three attention theories (three rows). Cue and stimulus diameters were $1.4^{\circ}$ and $1.9^{\circ}$, respectively (drawing approximately to scale). For clarity, cue stimuli (small disk outlines) are indicated by asterisks. The movement of attention according to the shifting model is illustrated by showing the location of focused attention in the display at two time points, denoted $t_{x}$ and $t_{x+1}$. For illustration, a fixed arrangement of standard target, variable target, and distractor was chosen, but in the experiment the position of the stimuli was randomized for the shown triangular configuration, and also a top-down triangular configuration was used.

responses at a zero SOA, indicating that the prior entry for the variable target equaled that for the standard target. Second, in a single-cue condition cuing the variable stimulus, prior entry was induced for that stimulus, shown by a shift of the psychometric curve to the right (e.g., less than 50\% "standard before variable" responses at a zero SOA). Third, in a target-target cuing condition, the targets were both cued, again leading to no difference in prior entry between variable target and standard target. In a fourth condition (target-distractor cuing), the variable target was cued as well as the distractor. This resulted in a prior-entry effect for the variable target that was not different from that seen in the singlecue condition (expressed in time, it equaled $30-40 \mathrm{~ms}$ in both conditions). Scharlau (2004) argued that this suggested a split of attention between the distractor and the variable target. According to a serial shifting variant of the unitary focus theory, random assignment or shifting of the focus between the cued distractor and the cued variable target would have led to a reduction of the prior-entry effect for the variable stimulus in the target-distractor cuing condition compared with the single-cue condition, but this was not observed.

A second experiment (see Figure 4) showed that in the variable target-distractor cuing condition, it did not matter whether the standard was positioned away from the variable target and distractor (standard away condition) or in between (standard in-between condition). In the two conditions, the prior-entry effect of the variable stimulus relative to the standard was the same. Scharlau
(2004) argued therefore that the results excluded a zooming model and on the whole supported the existence of divided attention.

Despite the suggestive data, their interpretation is limited by several factors. First, a critical aspect of Scharlau's (2004) findings is that the prior-entry effect following cuing of the variable target stimulus remained unchanged when an additional (distractor) stim-

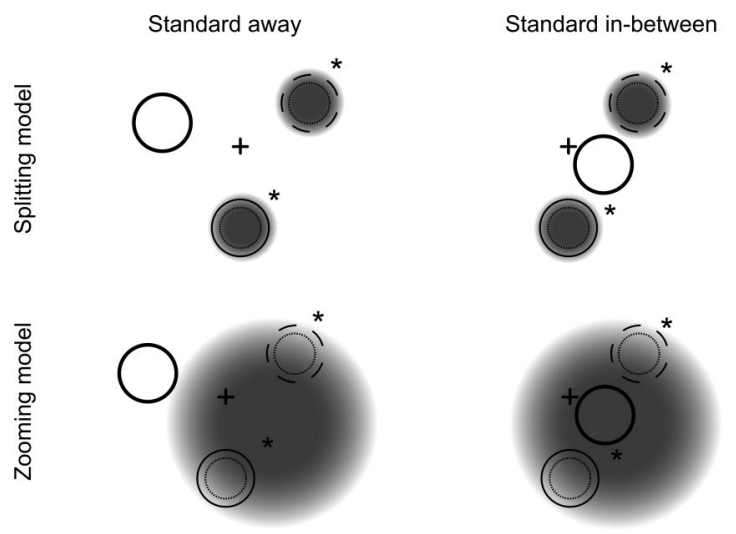

Figure 4. Experimental conditions used by Scharlau (2004) to test zoom lens and splitting models. Grayscale gradients indicate attention distributions after cuing variable target and distractor according to splitting model (top row) and zooming model (bottom row). Not to scale; other conventions as in Figure 3. 
ulus was cued. Thus, in this paradigm, the hypothesized division of attention did not reduce the amount of attention available in individual foci. This contrasts with other studies (Castiello \& Umiltà, 1992; McCormick et al., 1998) in which a division of attention over increasing areas of visual space (by increasing size or number of target locations) reduced the effectiveness of attentional processing. We suggest that the lack of a decrease in attentional enhancement for the variable target when also a distractor was cued shows that this dual cuing condition was far from draining attentional capacity. In light of an almost complete lack of processing requirements in different locations (participants needed to report only whether one stimulus appeared before the other), we argue that the task used in Scharlau was insufficiently difficult to tax attentional resources, which reduces the plausibility of a conclusion of divided attention (Criterion 1). Second, given the lack of processing requirements, the possibility remains that attention "hopped" between locations without much cost, thereby producing prior-entry effects in different locations. This hypothesis is not incompatible with the magnitude of the prior-entry effect, estimated to be in the order of $40 \mathrm{~ms}$ in Scharlau's study. Third, the test of the zoom lens model of attention in Experiment 2 by placing the standard stimulus in between cued stimuli was insufficient to exclude the possibility that attention was allocated flexibly to a unitary region in the visual field that avoided the location in between cued stimuli (Criterion 4). Fourth, and most fundamentally, there is no evidence in Scharlau's experiments that attention was ever directed to the distractor stimulus. For each participant, the standard target, variable target, and distractor had a fixed color throughout the experiment. In addition, the positions of the stimuli were randomized, and participants had to rely entirely on the color of the stimuli to identify the targets and the distractor (see Scharlau, 2004, pp. 990-991). Therefore, the selection of the targets was probably based on a parallel feature-based selection of the target colors across visual space and a suppressed processing of the distractor color. It seems a plausible possibility that prior-entry cues were effective in regions of visual space that were occupied by an attended color and not effective in regions of visual space occupied by a nonattended color. In exogenous cuing studies, similar conditional effects of cuing have been demonstrated (e.g., Folk, Remington, \& Johnston, 1992). Hence, we suggest that the cuing of the distractor was not effective (Criterion 3). This hypothesis explains why in Experiment 1 it did not matter whether, together with the variable target, the distractor was also cued and why in Experiment 2 it did not matter whether the standard target was placed in between cued variable target and cued distractor. Thus, it is likely that the cuing used by Scharlau was insufficiently effective to capture attention in locations that were not already cued by features of the target stimuli (Criterion 3 ).

In conclusion, although at face value the present experiment suggests the existence of parallel enhancement of processing in noncontiguous locations induced by prior entry, the question remains whether prior entry was an effective attentional modulation in the context of Scharlau's (2004) experiments. Even if it was, several plausible explanations of the observed results exist that are compatible with a model of unitary rather than divided spatial attention.

Single-target detection in complex stimulus arrays (endogenous cuing). Gobell, Tseng, and Sperling (2004) reported an "ability of spatial attention to conform to requested disjoint distri- butions" (p. 1293). In their experiment, participants had to report the location of a target stimulus in target regions and ignore distractors in distractor regions, as defined by a colored grating that was presented prior to the display with target and distractors (see Figure 5). Distractors were small white disks $\left(0.23^{\circ}\right.$ diameter), and the target was a slightly larger disk $\left(0.34^{\circ}\right.$ diameter $)$. Participants had to be trained in a session similar in length to the experimental session before the participants satisfactorily performed the task (1,700 trials). Eye movements were not monitored.

The instruction grating was a square-wave red-green grating covering the entire computer monitor $\left(18^{\circ} \times 13.5^{\circ}\right.$ at viewing distance of $120 \mathrm{~cm}$ ). On a trial-by-trial basis, orientation (horizontal or vertical), spatial frequency (approximately 0.06, 0.11, 0.17, $0.33 \mathrm{c} /$ degree), and phase shift of this grating were varied. The instruction grating remained present for $150 \mathrm{~ms}$ and then faded into a gray background over the next $450 \mathrm{~ms}$. After an additional $200 \mathrm{~ms}$, the search array was presented for $150 \mathrm{~ms}$ (unmasked). The search array consisted of a $12 \times 12$ array of disks (separated from one another by approximately $1^{\circ}$ and slightly jittered). The observer was instructed to report the position of the target in an array location within one of the attended patches of the screen. At the same time, the observers had to ignore distractors and/or false targets (1 or 10) in the to-be-ignored patches of the screen.

A response was considered correct when the target was localized in the correct location or a neighboring location. Performance declined from about $75 \%$ correct at a spatial frequency of 0.06 c/degree to about $25 \%$ correct at 0.17 and 0.33 c/degree (chance performance ranging from $2 \%$ to $8 \%$ in different conditions). This would correspond to a performance decrease from about $92 \%$ correct to about $78 \%$ correct if chance level had been $50 \%$, which indicates that the task was fairly easy, and approached a reasonable difficulty level only at the higher spatial frequencies $(0.17$ and 0.33 c/degree; Criterion 1). It is interesting that decreasing viewing distance to $60 \mathrm{~cm}$ (and thus decreasing spatial frequency by a factor of two) led to results indistinguishable from those obtained at $120 \mathrm{~cm}$. Gobell et al. (2004) also showed that performance depended strongly on the exposure time of the instruction grating, with performance starting to decline for exposures of less than $350 \mathrm{~ms}$.

Gobell et al.'s (2004) results may be indicative of a form of divided attention with a fairly low spatial resolution. It can be questioned, however, whether the allocation of attention was based on a purely spatial process. The attention distribution was set up by attending to a feature (green or red) in the instruction grating, and it was the short-term memory of this colored grating pattern that guided the distribution of attention. Short-term (working) memory for objects and their features can modulate feature processing in visual areas (Chelazzi et al., 1993) in parallel across visual space (Bichot et al., 2005; Boynton, Ciaramitaro, \& Arman, 2006; Motter, 1994a, 1994b; Sàenz, Buracas, \& Boynton, 2003). Therefore, working memory may be the source of feature-based gating of processing of the relevant area in the instruction grating, possibly through some interaction between spatial and feature- or objectrelated mechanisms in prefrontal cortex (Rao, Rainer, \& Miller, 1997). The dominant contribution of object-related mechanism in setting up the attention distribution in Gobell et al. stimuli is supported by the finding that a change in viewing distance did not affect the decline of performance with increasing numbers of increasingly narrow patches in the instruction grating. Therefore, 


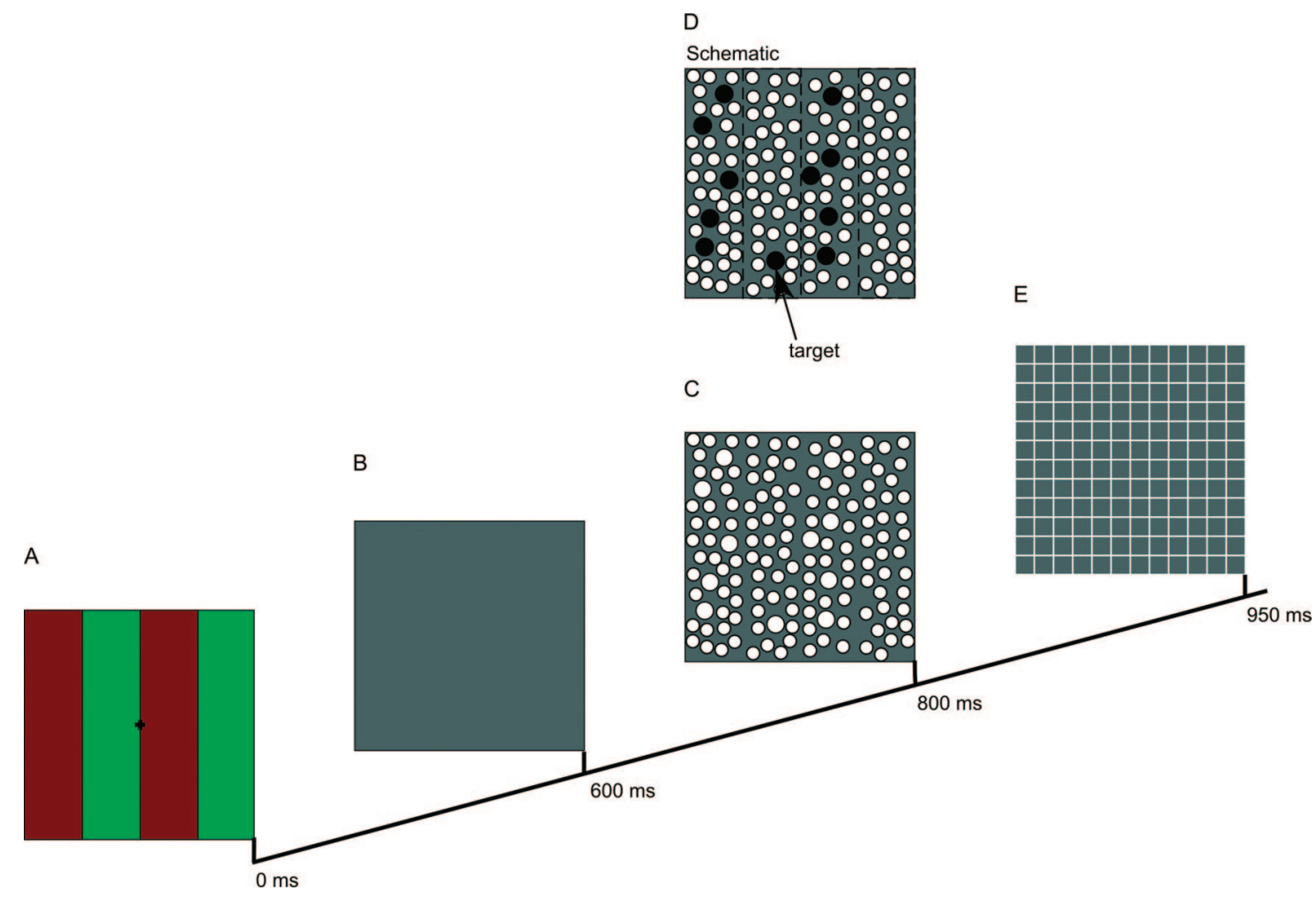

Figure 5. Overview of the stimulus sequence in the study of Gobell et al. (2004). (A) Participants were presented with an instruction grating and had to attend the patches in one of the two colors defining the instruction grating. In the stimulus illustrated here, participants would have been instructed (verbally) to attend to the green patches. (B) After $150 \mathrm{~ms}$, the instruction grating started to fade out into a gray screen (450-ms fading period). (C) Subsequently, a position-randomized $12 \times 12$ array of small disks was presented. The array consisted of 133 small distractor disks and 11 slightly larger disks. Ten of the larger disks were randomly distributed over the areas that had to be ignored (corresponding to the red regions in the instruction grating), and they are referred to as false targets. A single larger disk was shown in one of the areas that had to be attended (corresponding to the green regions in the instruction grating) and is a true target. The task of the participants was to identify the position of the target disk while ignoring false targets (in some experiments false targets were removed). (D) The target and false targets are shown in black, and the separation between attended and to-be-ignored regions is shown in dashed lines for illustrative purposes. (E) Finally, a response display was shown in which the participant had to indicate the position of the target. From trial to trial, the orientation and spatial frequency of the grating were randomized.

for the range of frequency conditions chosen, the decline did not depend on retinal spatial frequency but rather on the size of the patches of the instruction grating on the screen irrespective of viewing distance. This is a strong indication that the obtained attention effects were primarily object related rather than spatial. Possibly, the working memory mechanisms in prefrontal cortex that determined sensory gating of processing made use of sizeinvariant object-related information arriving via inferotemporal cortex (Lueschow, Miller, \& Desimone, 1994). Therefore, we contend that Gobell et al. revealed primarily an object-related effect of attention, rather than a spatial effect in a retinotopic coordinate system (see Table 1, Criterion 3).

Beyond the general question whether the attentional manipulation in this study was one of spatial attention, some limitations in interpreting the study arise from the unmasked 150 -ms presentations of the search arrays (Criterion 2) and the long cue-to-target intervals (>350 ms; Criterion 3). In light of these parameters, it cannot be excluded that participants prioritized processing of one of the attended areas and then attempted to increase performance by shifting attention within the 150-ms exposure (unmasked) to another region.

Gobell et al. (2004, Figure 2b) showed a "landscape" of performance correct for the entire $12 \times 12$ array, averaged over attention conditions. In two of the three observers shown, the data revealed a strong decline of performance correct with distance from the fovea. It is unfortunate that the investigators did not split their data to show the attentional landscapes for separate orientations, to demonstrate that the orientations of regions of enhanced performance coincided with attended regions as cued by the instruction grating. In addition, it is unfortunate that there was no detailed measure of attention in to-be ignored stimulus regions. However, Gobell et al. did report a strong increase of performance when false targets were removed from "unattended" areas. This suggests interference by the false targets in the unattended areas, which 
normally is considered evidence against the splitting of attention (Criterion 4). In conclusion, although the design and stimulus display used by Gobell et al. are promising, further experiments are necessary to show that the conclusion of divided attention is warranted and applicable to the domain of spatial attention.

\section{Two-Target Discrimination and Identification Experiments}

Two-target matching experiments with sudden-onset and no-onset stimuli. Kramer and Hahn (1995) tested the possibility of a noncontiguous division of the spotlight of attention using a two-target matching experiment. Two target letters (cued by frame stimuli) were presented together with two distractor letters in between, and the task of the participant was to indicate whether the target letters were identical. The frames were presented mirrorsymmetrically around the vertical meridian in the upper or lower quadrants of the visual field, above or below fixation. The two target letters were presented inside the frames $150 \mathrm{~ms}$ after onset of the frame cues, together with the two distractor letters in between the target locations (cue-to-target interval $150 \mathrm{~ms}$ ). The display with letters remained present briefly (60 ms), and letters were masked. Eye fixation was not monitored. The four letters were separated by about $2.5^{\circ}$ and had the same eccentricity of $8.5^{\circ}$. The cue provided by the frame had an exogenous component (related to its onset $150 \mathrm{~ms}$ before stimulus onset) and an endogenous component (related to the expectation of the targets within the frames, which were always presented in the same two locations).

The four letters were presented as either "sudden onset" stimuli (presented at once on a blank screen) or "no onset" stimuli. The no-onset stimuli were "digital eights" (the number 8 consisting of seven line segments) presented together with the frame cues in the locations of the target and distractor stimuli, and modified after $150 \mathrm{~ms}$ into letters by removing segments of the digital eight. For example, the digital eight became an "E" by removing two segments. The no-onset stimuli were introduced to remove exogenous effects associated with sudden-onset stimuli. Kramer and Hahn (1995) reasoned that onset effects of stimuli shown in between target stimuli automatically attract attention, unfairly suggesting that attention cannot be split. The rationale behind the use of no-onset stimuli was to remove this confound.

Split focus and zoom lens models of attention make different predictions about the processing of the distractor letters in between cued locations. According to the splitting model, the identity of distractor letters would not influence target-target matching responses, but according to the zooming model it would. According to the zooming model, a participant's attention would be directed not only to the targets but also to the in-between distractors. A distractor matching one of the targets would therefore facilitate a match response independently of whether or not the targets matched, and a nonmatching distractor would facilitate a nonmatching response regardless of whether targets matched. Thus, the present study made use of distractor interference on target performance to assess the distribution of attention. Note that the brief presentation times paired with masking made any type of attention reallocation during and immediately after stimulus presentation unlikely.
In the sudden-onset condition, there was an interaction between target-target matching and distractor-target matching status. When matching distractors were present, target-target matching responses were about $25 \mathrm{~ms}$ faster and $3 \%$ more accurate than target-target mismatch responses. When distractors were nonmatching with the targets, target-target matching responses were about $30 \mathrm{~ms}$ slower and 10\% less accurate than target-target mismatch responses. By contrast, in the no-onset condition, reaction time and accuracy in the target matching task did not depend on the matching or nonmatching status of the distractors, and in fact, results were indistinguishable from data obtained without distractors. This indicates that participants could ignore the distractors in the no-onset condition, but in the sudden-onset condition they could not. Irrespective of distractor status, reaction times for matching targets were about $50 \mathrm{~ms}$ slower, and performance was about $15 \%$ lower, compared with nonmatching targets. Very similar results were found when the cue-to-target interval was increased to $600 \mathrm{~ms}$ and when all stimuli were confined within a hemifield (Hahn \& Kramer, 1998).

At first sight, the results in the sudden-onset condition agree with the zooming model, and the results in the no-onset condition with the splitting model. However, it cannot be excluded that a zooming account could apply to both no-onset and sudden-onset conditions. In the no-onset condition, there is the untested but plausible possibility that a narrow elliptical zoom assumed a shape to include only the target locations and to avoid the distractor locations (Criterion 4). This may have been facilitated by the equieccentric arrangement of targets and distractors (distractors being slightly offset from a straight line connecting the targets). In the sudden-onset condition, the endogenous setup of the attentional zoom lens to avoid distractors might have been overruled by or complemented with the bottom-up exogenous cues associated with the onset of all four stimuli. This is plausible given the rapid time course of exogenous cuing effects, which take place before endogenous cues can become dominant (see, e.g., Steinman \& Steinman, 1998). Furthermore, reaction times were around $550 \mathrm{~ms}$ in the sudden-onset condition, which makes it plausible that exogenous cuing effects still determined the distribution of attention while participants made their target-target matching decisions. Hence, the elliptical focus of attention might have included all target and distractor locations in the sudden-onset condition due to exogenous cues associated with the sudden onset of the stimuli.

That exogenous cues can override task-related distributions of attention has been reported in both human psychophysical studies (Donk \& Theeuwes, 2001; Enns, Austen, Di Lollo, Rauschenberger, \& Yantis, 2001; Franconeri \& Simons, 2005; Jonides \& Yantis, 1988; Theeuwes, 1995) and monkey neurophysiological studies (Balan \& Gottlieb, 2006; Kusunoki et al., 2000). This occurs for various types of exogenous cuing (abrupt onsets of new stimuli, as well as color and luminance changes of existing stimuli) and with little spatial selectivity. Furthermore, whether exogenous cues dominate endogenous cues depends on experimental conditions. Folk et al. (1992) claimed that salient distractors capture attention only if they share task-relevant features with the target stimulus. In line with these findings, Bacon and Egeth (1994) showed that the effect of attentional capture was diminished if the relevant target dimension was unknown to the participants at the time of cuing. Belopolsky, Zwaan, Theeuwes, and Kramer (2007) showed that salient distractors capture attention only if they are 
encompassed by the endogenous zoom lens of attention. Taken together, these studies show that attentional capture is most likely within the zoomed region of attentional enhancement for stimuli that share task-relevant features with the target. We suggest that in Kramer and Hahn (1995), immediately following cuing, a coarse, unified attention distribution was set up to avoid distractor locations but that the onset of four stimuli at the edge of the attention distribution contained enough energy to shift or expand the distribution of attention to include distractor locations. In other words, shortly after stimulus onset, exogenous and endogenous factors may both contribute to the momentary distribution of attention in a single contiguous region of the visual field. Further, the same type of stimulus material was used for target stimuli and distractors, and the distractors were explicitly chosen so they could be judged in a manner similar to the letter-matching task performed on the targets. These experimental conditions are likely to enhance involuntary processing of the distractors. Hence, the data from Kramer and Hahn seem compatible with a hypothesis of a single contiguous region of attentional enhancement that is positioned away from distractors for no-onset stimuli but drawn toward distractors for sudden-onset stimuli. Note that in the sudden-onset condition, reaction times were about $200 \mathrm{~ms}$ faster than in the no-onset condition, which is compatible with the idea that fast exogenous cuing effects contributed to faster attentional processing, which, however, involved both the distractors and the targets.

At first sight, the reasoning that exogenous cues directly associated with stimulus onset can override task-related attention may make any divided attention experiment impossible. In many experiments, targets presented in an unexpected location (probing stimuli) are used to measure the amount of attention near the target locations. If the onset of a probing stimulus always overrides endogenous attention, then the distribution of attention prior to the probing stimulus cannot be tested. However, the effects of exogenous cuing and endogenous cuing are competitive (e.g., Bell and Munoz, 2008), and which cue will win will depend on the relative strength of the cues (e.g., Bacon \& Egeth, 1994; Folk et al., 1992). In most experiments using single probing stimuli, it can safely be assumed that a weak probing stimulus (often a dot or a target letter) will not (completely) override existing or developing distributions of endogenous (task-related) attention incorporating the target locations. However, in Kramer and Hahn (1995), there is a competition between the attention that is directed to the target locations by preceding cues and the large exogenous cue associated with the simultaneous onset of four stimuli (two targets and two distractors). In that experiment, it seems reasonable to hypothesize that exogenous cuing effects due to the simultaneous onset of four stimuli override prior allocations of attention.

The effects of exogenous cuing resulting from stimulus onset may be avoided not only by using no-onset stimuli, as done by Kramer and Hahn (1995), but also by using tasks that are difficult enough to ensure that participants take their perceptual decisions long after the transient effects of the exogenous cues have vanished. Note that in Kramer and Hahn these two factors cannot be separated from each other. With the no-onset stimuli, accuracy was about $10 \%$ lower and reaction times were about $200 \mathrm{~ms}$ longer than with sudden-onset stimuli, which suggests that the loss of distractor interference in the no-onset condition could have been an effect of both the lack of onset transients and increased difficulty. Further, the high level of performance in no-onset $(80 \%$ correct) and especially sudden-onset conditions (90\% correct) incurs doubts about the suitability of the difficulty level of the tasks used in Hahn and Kramer to test divided attention (Criterion 1).

In another experiment aimed at finding support for parallel processing in separate foci of attention, Hahn and Kramer (1998) extended the experiment of Kramer and Hahn (1995) by including a manipulation of stimulus quality, using otherwise similar displays and timing parameters. Displays were presented containing two target letters that were both of low quality $(L L)$, both of high quality $(H H)$, or of low and high quality $(H L)$, and the stimuli were presented with either a sudden onset or no onset. A serial model predicts that total processing time would be the summation of the processing times for the individual stimuli, with the longest processing time for two low-quality visual stimuli $(L L>H L>H H)$. A parallel model predicts that total processing time equals the processing time of the stimulus that requires the longest processing time $(L L=H L>H H)$. The reaction time results with suddenonset stimuli appeared to support the serial model, whereas results with the no-onset stimuli appeared to support the parallel model. Further, two distractors placed equieccentrically between targets did not influence target-matching responses in the no-onset conditions, but they did in the sudden-onset conditions. Hahn and Kramer concluded that in the no-onset condition, the data are compatible with a parallel division of attention. However, just as in Kramer and Hahn, the possibility remains that in the no-onset condition, a zoomed region of attentional enhancement was set up to avoid the two distractors and to optimize performance at target locations (Criterion 4). Hahn and Kramer's study, though suggestive, is not a conclusive demonstration of divided attention.

Bichot, Cave, and Pashler (1999) performed another location cuing experiment to reinvestigate the possibility of splitting the focus of attention. In each trial, eight shape outlines were presented at $6.1^{\circ}$ of eccentricity around a fixation spot, two of which were distinct in color and served as target regions (see Figure 6). The outlines were presented for $105 \mathrm{~ms}$, after which a letter was placed inside each outline for $60 \mathrm{~ms}$, upon which the display was masked. The participants had to report whether the two targetoutlines matched in shape and also had to report as many letters as possible. Eye fixation was not monitored. On the shape-matching task, participants reached a performance of $85 \%$ correct for adjacent shapes, with a small but significant drop for nonadjacent ones (80\%). Within correctly discriminated outlines, participants correctly identified up to $40 \%$ of the letters. Virtually no correct identifications took place within distractor outlines, and this held true even when only a single distractor outline was positioned in between two target outlines.

Bichot et al. (1999) took their data as support for the split focus model of attention. Specifically, they interpreted the absence of correct letter identifications in distractor regions between target locations as evidence against the zoom lens theory and against serial movements of a focus of attention. However, a simple unified attention distribution (e.g., resembling a narrow, elliptical zoom) could have formed to include target locations, excluding in-between locations. The stimulus arrangement used (Criterion 4) therefore does not permit a conclusive interpretation of the data in favor of divided attention. A study by Dubois, Hamker, and VanRullen (2009) replicated the findings from Bichot et al. using the same circular stimulus arrangement, but also established that attention was present at the target letters only for $100-150 \mathrm{~ms}$, 


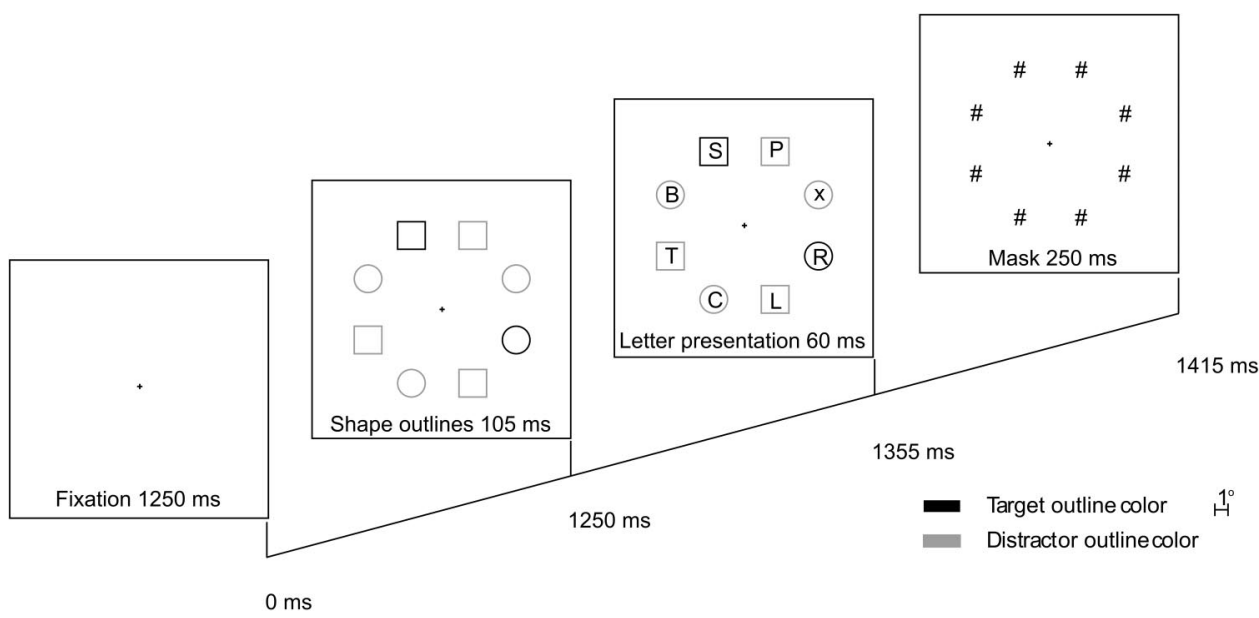

Figure 6. Design and stimuli used by Bichot et al. (1999). Sequence of stimulus displays is illustrated from left to right. After fixation $(1,250 \mathrm{~ms})$, eight empty shape outlines (circles and squares) were presented $(105 \mathrm{~ms})$ at an eccentricity of $6.1^{\circ}$. Subsequently, a letter was placed in each outline $(60 \mathrm{~ms})$ and masked $(250 \mathrm{~ms})$. Participants compared the shapes of two target outlines that differed in color from the others (target outlines illustrated in black and distractors in gray) and also reported as many letters as possible.

after which attention became focused on one or the other target stimulus. Dubois et al. interpreted their findings as support for divided attention but also indicated that divided attention is unstable. However, because Dubois et al. replicated the stimulus conditions used by Bichot et al., it is equally likely that a unified, elliptical focus of attention was allocated to the region occupying the targets, while avoiding distractors, and that within $150 \mathrm{~ms}$ attention shifted to one or the other target. In essence, the experiment by Dubois et al. is subject to the same criticisms as the comparable experiment in Bichot et al.'s article (Experiment 6), as the experimental design was unchanged.

Two-target identification experiments using complex stimulus arrays. The experiments discussed in the preceding sections used displays with a fairly limited number of items. Awh and Pashler (2000) performed several experiments to test how attention could be divided among potential target locations in more complex displays with large numbers of distractors (see Figure 7). The hypothesis behind these experiments is that a true division of attention is more likely if in between (and around) the target locations, a large number of distractors have to be actively ignored.

In Awh and Pashler's (2000) experiment, participants fixated in the middle of a screen when presented with two cuing stimuli (equal signs, presented for $750 \mathrm{~ms}$ ) that marked the positions where target numbers (two digits) would appear (see Figure 7B). Immediately following these two cues, a $5 \times 5$ array containing 23 letters and two target numbers was shown, centered on the fixation spot $(118 \mathrm{~ms}$, with some variability between participants as exposure was adapted to performance). Eye fixation was not monitored. Only the four corners of the central $3 \times 3$ elements in the array could contain target digits, pairs of which could be cued. On $80 \%$ of the trials, the two positions where a digit could appear were cued validly; on $20 \%$ of the trials, the positions were cued invalidly. When the target positions were cued invalidly, one target digit appeared in a location between cued locations and the other in a second position further away from cued locations. The invalidly cued trials had the purpose of probing target processing in distractor locations. For example (Figure 7B1), after cuing the top left and top right position of the central $3 \times 3$ array, targets could be presented in the cued positions (valid trial) or in the middle positions of top row and bottom row. After the presentation of the array (Figure 7C), all characters were masked (Figure 7D). After the mask, an instruction display was given to the participants to indicate the two (cued or uncued) locations in the array for which a target report was desired (see Figure 7E). The accuracy of the participants' report was measured in two locations indicated by a question mark. Note that the global, imaginary orientations defined by targets in valid and invalid trials were orthogonal. Further, cuing was directed at two positions in different hemifields (Figures 7B1 and 7B4) or at two positions in the same hemifield (Figures 7B2 and 7B3).

The accuracy data for horizontal cuing showed that on invalidly cued trials, accuracy of target reports for the invalidly cued positions $(<35 \%)$ decreased strongly compared with accuracy for validly cued positions $(>85 \%)$. This may suggest that processing of the cued locations was enhanced, while the region in between was ignored, in agreement with a splitting model of attention. This interpretation is less straightforward than it seems, however, as the requirement in invalid trials to report on an extra unattended item in addition to the item in between attended locations puts the in-between item at an extra disadvantage compared with the inbetween and the attended items. The extra item is located as far away from the targets and in-between item as the distance between the targets themselves. Although processing of the far target appears to have been ineffective $(10 \%-15 \%$ correct), the mere requirement to report on the far item may have interfered with reports on the in-between item. Hence, the strategy of validly or invalidly cuing pairs of locations and asking reports on pairs of locations may have led to an underestimate of processing at the in-between distractor location (Criterion 3). We suggest that invalid cuing of single distractor locations chosen unpredictably from multiple locations in between and surrounding targets might have been a more effective manner of probing attentional distri- 


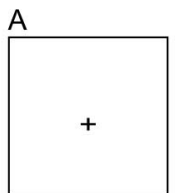

B1

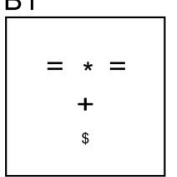

B2

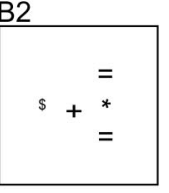

B3

$$
=
$$$$
\text { * }+s
$$

B4

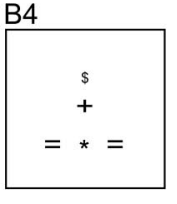

$750 \mathrm{~ms}$

$500 \mathrm{~ms}$
C1

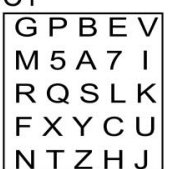

$\mathrm{C} 2$

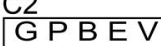

MA 5 W I

R Q S L K

$\mathrm{F} \times 7 \mathrm{C}$

NTZH J

D

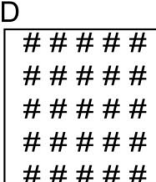

\#\#\# \# \#

E1

? ?

. . . .

E2

- .

.. . .

. ?.

stimulus duration

trial time

$0 \mathrm{~ms}$

$500 \mathrm{~ms}$

$1250 \mathrm{~ms}$

$\pm 1368 \mathrm{~ms}$

$\pm 1468 \mathrm{~ms}$

until

subjects'

response

Figure 7. Trial overview and stimulus layout used by Awh and Pashler (2000) in their Experiment 1. (A) Participants fixated a cross in the middle of the screen for $500 \mathrm{~ms}$. (B) After the fixation period, two locations where a target was likely to occur were cued by equal signs for $750 \mathrm{~ms}$. Four cue arrangements were used by Awh and Pashler. The middle and far uncued positions are depicted as asterisks and dollar signs, respectively. (C) After the cue period, a $5 \times 5$ array of alphanumeric characters was shown, with 23 letters (distractors) and two numbers (targets). The presentation duration of the character array was adapted to the performance of the participants. Note that B1 contains valid cues for stimulus display C1, as the number targets fall within the cued locations. By contrast, the cues in B1 are invalid for stimulus display C2. Arrays C1 and C2 are associated with B1; stimulus arrays corresponding to B2-B4 are not shown. (D) After the stimulus display, a $5 \times 5$ array of hash signs was presented to mask the stimulus display (mask duration $=100 \mathrm{~ms}$ ). (E) Finally, participants were presented with a response display, and they had to type the numbers they had perceived at the location of the question marks. The next trial was initiated after the participants gave a response.

butions around the target locations (Criterion 4). Altogether, though suggestive, the outcome of this experiment does not unambiguously support the idea of divided attention.

Furthermore, participants in Awh and Pashler (2000) went through a 120-trial training procedure in which the stimulus exposure duration was optimized $(X=118, S D=39 \mathrm{~ms})$ to reach a satisfactory performance level. This is a somewhat risky procedure, as it may permit participants to choose attentional strategies. Cue-to-target interval was $750 \mathrm{~ms}$, and it is not impossible that participants prioritized a single target location to be processed first and then moved attention to the second target location during stimulus presentation (Criterion 3). Hence, it is plausible that a number of participants used a sequential reallocation of attention during stimulus presentation to optimize performance.

Awh and Pashler (2000) repeated the experiment without distractors (but with masking in all $5 \times 5$ array positions) and also with neither distractors nor masking stimuli (hash signs). In these two experiments, the decrease in performance for uncued targets was far less pronounced or absent. Awh and Pashler suggested that this revealed active suppression of distractors when they were presented in between attended locations (as in the previous experiment). However, it is equally plausible that the (serial) reallocation of attention that is required to attempt reporting on targets in uncued locations is facilitated by the absence of factors that could interfere with attentional reallocation (i.e., the presence of other stimuli and masks).

Awh and Pashler (2000) also reported that in the difficult version of their experiment (with distractors and masking), the accuracy of validly cued targets was higher when they were presented in different hemifields (horizontal cuing; see Figures 7B1 and 7B4) compared with when they were presented in the same hemifield (vertical cuing; see Figures 7B2 and 7B3). The disadvantage for targets in the same hemifield disappeared in the easier versions of the experiments without distractors and without masking. Thus, when the task was harder, attentional capacity limits were reached earlier for within-hemifield targets than for across-hemifield targets. At face value, this finding suggests that when two targets are placed in two hemifields, more 
attentional resources become available to process them. However, this does not answer the question whether the processing of the stimuli proceeded in parallel foci of attention or through a dynamic reallocation of attention (for a discussion, see next section). In conclusion, the hypotheses formulated by Awh and Pashler that attention can be truly divided between two targets, and that this entails active suppression of in-between stimuli, needs further testing.

Two-target matching experiments with intra- and interhemispherically placed targets. Kraft et al. (2004) noted that in many studies of divided attention, the division of resources over two specific locations is tested between locations in opposite hemifields (see Table 1), thereby confounding the issue of crosshemispheric attention with the issue of divided attention in general. Taking into account evidence from split brain patients (Arguin et al., 2000; Holtzman, 1981), as well as the general contralateral organization of the visual system and attentional control structures, Kraft et al. hypothesized that divided attention might be more easy to accomplish across hemispheres than within. In line with that idea, studies in which participants were required to report on two target items revealed better performance when the two targets were presented in opposite hemifields rather than within a single hemifield (Awh \& Pashler, 2000; Banich, 1998; Brown \& Jeeves, 1993; Sperling \& Reeves, 1980). Kraft et al. also suggested that the latter finding is more robust for difficult than for easier tasks. On the basis of these observations, they designed an experiment in which the positioning of targets within or across hemifields was explicitly manipulated for different levels of task difficulty.

In the standard display used in Kraft et al.'s study (2004), four stimuli were presented (of which two were targets) in four locations indicated by frames. The first frame (Position 1) was located just above the horizontal meridian at $6.1^{\circ}$ of eccentricity. The second frame (Position 2) was placed in the lower left quadrant just below the horizontal meridian at $3.1^{\circ}$ of eccentricity. The third frame (position 3 ) was located in the lower left quadrant just to the left of the vertical meridian at an eccentricity of $3.1^{\circ}$. The fourth frame (Position 4) was positioned to the right of the vertical meridian below fixation at $6.1^{\circ}$ of eccentricity. With this arrangement, Kraft et al. could compare performance on targets cued in adjacent frames within hemifields (Positions 1 and 2) and in adjacent frames across hemifields (Positions 3 and 4). Likewise, they could compare performance on targets within a hemifield (Positions 1 and 3) separated by a distractor (Position 2) with performance on targets across hemifields (Positions 2 and 4) separated by a distractor (Position 3 ). The stimuli in the frames were simple, high-contrast shapes that were easy to discriminate (Task 1), symbols at high contrast that were more difficult to discriminate (Task 2), or simple shapes that were more difficult to discriminate because of low contrast (Task 3). Participants signaled with a button press whether the target stimuli were matching. Participants started fixating a central cross for $3 \mathrm{~s}$, after which the four frames were presented. Presentation of the frames was followed by a central cue that indicated the two frames where targets would be presented. The central cue remained in view (for 4, 7, or $10 \mathrm{~s})$ until the stimuli appeared (100 ms, unmasked). The frame and cue stayed in view during the response period. Eye fixation was monitored.

Here we compare performance on Task 1 and Task 2 for which the evidence in Kraft et al. (2004) most clearly supported that they were different in difficulty level. The average reaction time was $833 \mathrm{~ms}$ and the error rate $10 \%$ in Task 1 , which was significantly lower than the $935 \mathrm{~ms}$ and $20 \%$ in Task 2. Furthermore, reaction times and error rates were significantly larger for targets within a hemifield compared with targets across hemifields, indicating a bilateral field advantage. The most difficult condition was the hard version of the experiment (Task 2) with targets placed within hemifields (average performance was $75 \%$ correct, average reaction time was $967 \mathrm{~ms}$ ). The extent of distractor interference (increase of reaction time or increase of error rate in distractor condition compared with adjacent condition) was used as a measure for split attention.

We consider the results for targets placed within a hemifield first. In terms of reaction time, distractor-induced interference within a hemifield was $81 \mathrm{~ms}$ in the easy task and decreased to 41 $\mathrm{ms}$ for the difficult task. In terms of error rates, distractor-induced interference within hemifield was $8 \%$ in both the easy task and the hard task. Although not clear from error rates, the reaction time data suggest that making a task harder for targets within a hemifield reduced distractor interference, which can be seen as a trend toward splitting attention. However, considering all the experiments (five in total), a trend for distractor interference within hemifields remained invariably present across experiments (including the more difficult ones), and a combined statistical analysis might well have confirmed that. Although Kraft et al. (2004) did not perform this analysis, they concluded that a split of the attentional focus within hemifields may not be possible, even with tasks of appropriate difficulty level.

The results for targets across hemifields showed a slightly different pattern. In terms of reaction time, distractor-induced interference was $41 \mathrm{~ms}$ in the easy task and $40 \mathrm{~ms}$ for the difficult task. In terms of error rates, distractor-induced interference was $0 \%$ in the easy task and $1 \%$ in the difficult task. These data suggest that virtually independent of task difficulty, attention is split when the targets are presented in different hemifields. To explain the necessity for a difficult task to potentially split attention within a hemifield, Kraft et al. (2004) suggested that in easy tasks, there are sufficient resources available to process all stimuli at an early level, automatically leading to distractor interference. To explain split attention across hemifields, Kraft et al. suggested that "because of the increased attentional resources available when the attentional systems of both hemispheres are active, information can be processed more efficiently at an early level of visual processing, producing the bilateral field advantage" (p. 29). This suggestion, however, seems not to be entirely compatible with the idea that having sufficient resources (to process stimuli within a hemifield) in fact can prevent a division of attention. Implicitly, Kraft et al. assumed that the extra resources that become available when attending to targets in different hemifields are used separately in the two hemifields. If this view were correct, then the improved performance for those targets that were separated by a distractor when targets were presented across rather than within hemifields would be likely due to the fact that in one of the hemifields the target was never in competition with a distractor. Hence, a balanced design in which in two quadrants targets are presented surrounded by the same number of distractors would be better suited to study divided attention within and across hemifields. Furthermore, the idea that attention would be split between hemifields in healthy individuals goes against considerable evi- 
dence that visual processing at any point in the visual field draws on attentional resources from the two hemispheres. Neurons in high-level visual areas in the temporal lobe that contribute to attentional interactions across hemifields (e.g., Chelazzi et al., 1993) have large, bilateral RFs (Desimone, Albright, Gross, \& Bruce, 1984; Gross, Rocha-Miranda, \& Bender, 1972). These bilateral RFs reflect (splenial) cross-callosal and commissural connections (Gross, Bender, \& Mishkin, 1977). Furthermore, although for some specific functions either the left or the right hemisphere can be dominant (e.g., Mangun et al., 1994; Pouget \& Sejnowski, 2001; Sack et al., 2002; Weintraub \& Mesulam, 1987), parietal control of attentional allocation is based on a competition between hemispheres (Arguin et al., 2000; Holtzman, 1981; Larson \& Brown, 1997; Lomber, Payne, Hilgetag, \& Rushmore, 2002; Luck, Hillyard, Mangun, \& Gazzaniga, 1989; Arguin et al., 2000; Payne, Lomber, Geeraerts, van der Gucht, \& Vandenbussche, 1996; Sprague, 1996). Thus, the focus of attention might draw on attentional resources in both hemispheres depending upon a competition between hemispheres driven by task-related advantages of one or the other hemisphere (Brown \& Jeeves, 1993). The outcome of that competition may determine the relative contributions of the two hemispheres to the execution of a particular task. We speculate that for general aspects of attentional processing, such as the enhancement of sensitivity and other capabilities (Gopher, 1986; Kahneman, 1973; Navon \& Gopher, 1979; Wickens, 1980, 1984), both hemispheres contribute about equally and that the interhemispheric integration of attentional resources is more efficient along the vertical meridian rather than far away from it. This hypothesis is sufficient to understand why the presentation of targets shown across hemifields resulted in better matching performance: They were, on average, closer to the vertical meridian, whether or not the targets were separated by a distractor.

In sum, we suggest that in healthy observers, the integration of resources from the two hemispheres occurs within a single focus of attention. Placing stimuli in two locations in the two hemifields does not automatically give those stimuli access to spatially separate processing resources but, instead, may lead to more resources within a single region of attentional enhancement (and, we hypothesize, more so the closer the stimuli are placed to the vertical meridian).

Moreover, the hypothesis that the two targets can be processed with a single contiguous distribution of attention cannot be rejected with the arrangement of cued locations in Kraft et al.'s (2004) experiment, as the possibility exists that a curved or ringshaped distribution is set up to avoid interference by the distractors (Criterion 4). Kraft et al. suggested that their finding of better performance for across- than for within-hemifield targets is not likely to be compatible with shifts of attention, and they quoted evidence showing that reorienting attention within hemifields is easier than across hemifields (Hughes \& Zimba, 1987; Rizzolatti et al., 1987). However, other investigators could not replicate this finding (Henderson \& Macquistan, 1993; Macaluso \& Patria, 2007). Therefore, if the focus of attention could avoid distractors or could be turned off while shifting, there may have been enough time for attentional shifts both across and within hemifields. Kraft et al. agreed that shifts of attention "cannot be ruled out with certainty as the stimulus patterns were not masked" (p. 28; Criterion 2). A strategy of shifting attention might have been encouraged by the very long cue-to-target intervals, which, combined with the unmasked 100-ms exposure times, might have allowed participants to prioritize a single target location and then attempt to move attention to the other target location during stimulus presentation (Criterion 3). Kraft et al. emphasized the importance of the difficulty level of the task to demonstrate divided attention. In one condition (the within-hemifield condition in their Experiment 2A, with average reaction times of $967 \mathrm{~ms}$ and a performance level of $74 \%$ correct), the difficulty manipulation was successful, but in the other conditions of the different experiments, the tasks were fairly easy (Criterion 1). Hence, although many interesting topics were addressed in Kraft et al.'s article, including the important topic of task difficulty, the reported experiments do not provide strong evidence to suggest that spatial attention can be allocated simultaneously to multiple noncontiguous regions in the visual field.

Target locations cued by preprogrammed motor sequences. In most experiments of spatial divided attention, attention is covertly shifted to the relevant locations, either by instruction or by exogenous cuing. However, because of the close link between motor mechanisms and mechanisms directing attention (Klein, 1980; Rizzolatti, 1983; Rizzolatti et al., 1987), preparing a motor movement toward a location might be a useful technique to direct and control the location of covert spatial attention. Sheliga et al. (1994) demonstrated in human participants that the path of a saccade was influenced by the location of spatial attention. Furthermore, a number of studies have shown that the intention to perform a saccade to a particular location enhances perceptual performance at that location (e.g., Deubel \& Schneider, 1996; Kowler, Anderson, Dosher, \& Blaser, 1995). This finding is in line with studies in monkeys demonstrating attention-like enhancement of activity in V4 neurons (T. Moore \& Armstrong, 2003) and psychophysical enhancement of performance during microstimulation of the frontal eye fields (T. Moore \& Fallah, 2004).

If attention can be directed to a target location by making that location relevant for a motor movement, the question can be asked how spatial attention is allocated to several saccade target locations prior to a sequence of saccades. A number of studies have suggested sequential allocation of attention to multiple saccade goals (Henderson, 1992; Henderson, Pollatsek, \& Rayner, 1989; Morrison, 1984). Alternatively, when a task involves the execution of a predefined set of saccades, the entire sequence may be prepared as a whole, which might lead to a parallel allocation of attention to all relevant saccade targets prior to onset of the first saccade. Godijn and Theeuwes (2003) tested this idea in a series of experiments. In their paradigm, participants viewed an array of eight small disks of the same color arranged around fixation at an eccentricity of $8^{\circ}$; four disks were placed at the principal meridians $\left(0^{\circ}\right.$ and $\left.90^{\circ}\right)$ and four at the diagonal meridians $\left(45^{\circ}\right.$ and $\left.135^{\circ}\right)$. After a brief interval $(35,82$, or $129 \mathrm{~ms})$, a color change indicated the two saccade goals (target locations) on diagonal positions. In the following brief interval $(47 \mathrm{~ms})$, target letters were presented near the two saccade targets and near the two other diagonal positions (four in total). After the disappearance of the letters, the disk display remained in view for $800 \mathrm{~ms}$, and the saccades were executed in an order determined by the participant. At the end of the trial, a display with two letters was shown from which participants had to select the letter that had been shown at one of the two target locations. Godijn and Theeuwes reported that irrespective of the spatial arrangement of the target and nontarget locations, letter identification at target locations (77\% correct in Experiment 1) 
was always significantly better than at nontarget locations (58\% correct in Experiment 1). This held true also for nontarget locations in between target locations. These results were replicated when target locations were given by an endogenous (symbolic) cue at fixation and when the experimenter (rather than the participant) determined the order of saccades. Because letter identification was consistently better at the first ( $82 \%$ correct) compared with the second saccade goal (72\% correct), Godijn and Theeuwes considered it possible that a focus of attention was allocated to the first saccade goal in most trials and to the second saccade goal in a minority of trials. However, they showed that there was no difference in letter identification between conditions in which letters at target locations (as well as nontarget locations) were presented simultaneously or sequentially (Experiment 4). Additional data indicated that participants could match briefly presented $(77 \mathrm{~ms}$ ) targets presented simultaneously in the target locations (Experiment 5), at a performance level close to $80 \%$ correct. Godijn and Theeuwes interpreted their data as arguing in favor of a stable, parallel distribution of attention at the saccade target locations. In a similar study (Baldauf, Wolf, \& Deubel, 2006), attention was directed to multiple target locations by a sequence of hand movements, and very similar results were obtained. Baldauf et al. (2006) used a single-target identification design, but we briefly refer to this study in the present section on two-target experiments because in its concept of directing attention by means of motor preparation, the study is very similar to that of Godijn and Theeuwes. In both studies, it was concluded that attention was allocated in parallel to multiple noncontiguous-movement target locations prior to executing the programmed movement sequence.

The studies from Godijn and Theeuwes (2003) and Baldauf et al. (2006) are interesting demonstrations of the interaction between mechanisms of motor preparation and mechanisms of spatial attention. However, they do not unequivocally show that attention is spatially divided between the target locations. All the experiments by Godijn and Theeuwes and one of the experiments by Baldauf et al. involved two target locations that, together with distractor locations, were arranged equieccentrically around fixation. This stimulus arrangement and the large distances between neighboring stimuli would permit a single elliptical focus of attention to encompass the target locations (Criterion 4). The lack of a difference in letter identification performance between sequential and simultaneous conditions in Godijn and Theeuwes is in agreement with a single focus of attention encompassing the target locations, so there is no need to assume that spatial attention would be divided between target locations. The possibility of a single focus of attention set up to avoid locations or objects irrelevant for the prepared movements is strengthened by the traces of performed pointing movements in the two-target experiment of Baldauf et al. The observed pointing movements neatly avoided nontarget locations in between. It is possible that the movement traces, rather than the movement goals, correspond to the spatial allocation of attention. Baldauf et al. also performed triple-pointing tasks to allocate attention to three target locations, which would require a more complexly shaped distribution of attention if the targets were to fall within a single attentional focus (possibly along the movement traces). Even if this is considered less likely, the experiments from Baldauf et al. are subject to the criticism that the stimulus array was presented too long (170-220 ms) to prevent sequential reallocations of attention (Criterion 2) and that the task was insuf- ficiently difficult (86\% correct responses in Experiment 1; Criterion 1).

\section{Two-Target Experiments With Dynamic Displays}

Two-target identification in two RSVP streams. RSVP streams are often used to study the attentional blink, which is the suppression of processing during several hundreds of milliseconds following the detection of a target in a stream of stimuli (Chun \& Potter, 1995; Joseph et al., 1997; Potter, Chun, Banks, \& Muckenhoupt, 1998; Raymond, Shapiro, \& Arnell, 1992; Shapiro, Arnell, \& Raymond, 1997). However, it is also known that processing immediately following the target is relatively spared (Cousineau, Charbonneau, \& Jolicœur, 2006; Kawahara \& Yamada, 2006; Martin \& Shapiro, 2008; Zuvic, Visser, \& Di Lillo, 2000). Kawahari and Yamada (2006) investigated the properties of attentional allocation at the beginning of the attentional blink and interpreted their results as evidence for the ability to attend to two noncontiguous locations concurrently.

Kawahari and Yamada (2006) presented two RSVP streams, one $1.7^{\circ}$ to the left of fixation and the other $1.7^{\circ}$ to the right. The stimuli consisted of a series of digit distractors and occasional letter targets presented in rapid succession (100-ms presentations without interstimulus interval). Two targets were presented at the same time in the two streams ("first targets") and were followed by two more targets at intertarget intervals varying between 100 and $700 \mathrm{~ms}$ ("second targets"). Fixation was not monitored.

In agreement with previous literature on the attentional blink, Kawahari and Yamada (2006) found decreased performance in identifying the second targets when they followed the first targets too closely. Performance was worst at $200 \mathrm{~ms}$ after the first target and improved for target-target intervals greater than $200 \mathrm{~ms}$. Kawahari and Yamada also found that at the 100-ms-interval post-Target 1, there was a relative sparing of attentional processing. Specifically, they found that within the $85 \%$ of trials in which the two initial targets were identified correctly, $40 \%$ of trials also resulted in correct identification of both second targets. For the same 100-ms interval between first- and second-target pair, it was also demonstrated that participants identified only $25 \%$ of the two second-target stimuli when they were presented in between the standard locations of the RSVP streams (i.e., closer to fixation). The identification rate for the inwardly displaced second targets was significantly less than for second targets presented in the standard RSVP locations. No such effect was found for outwardly displaced second targets. These data were taken as evidence supporting divided spatial attention. Note that the inwardly and outwardly displaced second targets were masked within $100 \mathrm{~ms}$ by a digit.

The 100-ms stimulus durations and the masking by subsequent stimuli make shifts of attention during the simultaneous presentation of two targets unlikely. However, the claim of split attention applies only to the second targets presented $100 \mathrm{~ms}$ after the first targets; for all the other time lags (200-700 ms), there was no difference in performance between the inwardly displaced targets and the targets in their standard location. Furthermore, the evidence used to argue for split attention comes from a paradigm in which attentional processing is heavily suppressed. Kawahari and Yamada's (2006) claim of divided attention is entirely based on the somewhat weaker suppression at the standard RSVP (target) 
locations than at locations closer to the fovea during the first 100 $\mathrm{ms}$ following the first-target pair. The difference in suppression disappears for longer time lags. Thus, the claim of divided attention is based on the different time courses of the onset of suppression during the beginning of the attentional blink at peripheral target locations compared with locations on the horizontal meridian closer to fixation.

Even if one would take this as evidence for divided attention, that conclusion cannot be drawn with confidence. The stimuli were presented on a straight horizontal line through fixation, and it cannot be excluded that a unified region of suppression briefly morphed into a ring configuration of lesser suppression encompassing the target locations, rather than into two split distributions of lesser suppression during the first $100 \mathrm{~ms}$ of the attentional blink. Hence, the stimulus arrangement used was not suited to show conclusively either a split or a unified distribution of enhanced processing (Criterion 4), leaving any conclusion of how attention (or in this case, suppression) was distributed a matter of speculation. In addition, a transient modulation in suppression at the target locations during the first $100 \mathrm{~ms}$ of the attentional blink-even if it could be shown to be spatially limited to the target locations - can hardly be considered strong evidence for divided spatial attention. It would be an automatic phenomenon during the attentional blink, escaping control by the participant, and far removed from the idea that divided attention is an effective strategy that can be applied at will to efficiently process several simultaneously available sources of information.

Two-target comparisons during sequential and simultaneous target presentations in two RSVP streams. Shiffrin and Gardner (1972) suggested that a comparison of sequential and simultaneous target presentations could be highly informative in determining the contribution of serial or parallel processes. The main idea is that in a difficult task, the availability of only a single focus of attention would decrease performance if targets were presented simultaneously in different locations compared with presenting them sequentially. Bichot et al. (1999) tested this idea in an experiment in which participants fixated a fixation cross (fixation not monitored) in the middle of a screen while each trial presented a rapid succession of eight displays containing two digits (140 ms per display). The location of the two digits was fixed in each display, thereby creating two RSVP streams, one $2.5^{\circ}$ to the left and one $2.5^{\circ}$ to the right of fixation. In each RSVP stream, red digits were targets and green digits distractors, or vice versa (randomized over participants), and participants had to report the highest target number presented in the course of the eight displays. Emphasis was placed on accuracy; there was no time pressure. In the simultaneous condition, each of the eight displays contained either two targets or two distractors. In the sequential condition, a display showed a distractor on the left of fixation and a target on the right, or the opposite, and target and distractor were alternating on subsequent displays. Conditions were randomized, and within each condition the series started randomly with one or the other of the two possible displays (see Figure 8).

Bichot et al. (1999) found that the error rates for the sequential condition were in the same range as the error rates in the simultaneous condition, which supports divided rather than sequential allocation of attention. A weakness in the experiment, however, is that the task in the simultaneous condition was not very hard (Criterion 1). Performance in the simultaneous condition was close to $50 \%$, but this performance is the result of four individual
A

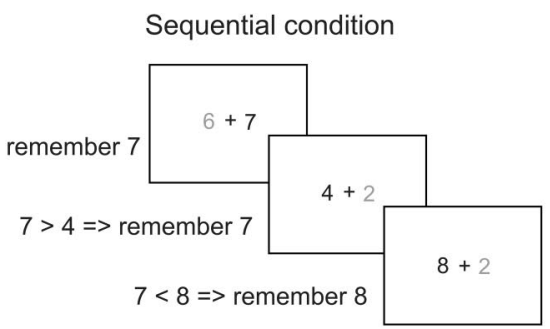

B

\section{Simultaneous condition}

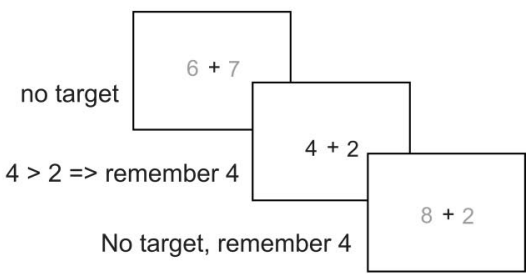

C

Shifting model

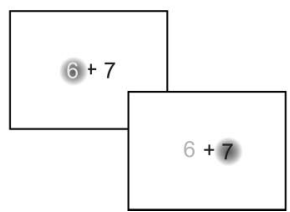

Splitting model

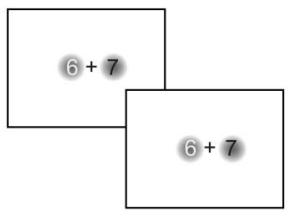

Zooming model

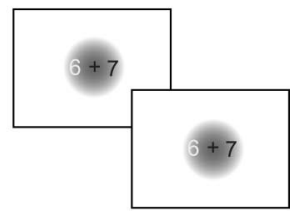

Figure 8. Overview of sequential and simultaneous conditions in experiments of Bichot et al. (1999) and summary of hypotheses derived from three attention models. (A) Sequential condition, in which participants had to report the highest target number that had occurred in a series of displays (three out of eight displays shown). Targets (illustrated in black) differed in color from nontargets (illustrated in gray). Cognitive operations are indicated to the left of each display. (B) Simultaneous condition, in which targets were paired within a display. Conventions as in Figure 8A. (C) Possible attentional distributions predicted from three models of attention (shifting, splitting, and zooming). Attentional modulation is indicated in gray (with black indicating stronger enhancement). Zooming lens models were tested by placing distractor stimuli at fixation and other locations in between the target locations (not illustrated). 
decisions taking place in the course of each trial. Individual decisions must have been correct at a rate of approximately $85 \%$ in order to reach a composite performance per trial of $50 \%$ correct, which makes the task insufficiently attention demanding in the simultaneous condition to expect great improvement in the sequential condition. The possibility exists that there was already sufficient time to process the two simultaneous targets with a serial mechanism, such that the increased processing time available for serial processing in the sequential condition would not constitute a significant advantage. In addition, it is unclear whether the errors made in this task were related to the processing of the targets or to the computations performed on the numbers. The computations themselves are attention demanding, and they might have been responsible for most errors. Because these computations are unlikely to be affected by the serial or simultaneous stimulus presentations, error rates might have been unaffected by these two conditions. Further, the sequential condition requires a comparison between a number held in memory and a currently perceived number. This makes the task more complex in the sequential than in the simultaneous condition, and given equal observed performance, the purely perceptual aspect of the task might have been carried out even more efficiently in the sequential condition than in the simultaneous condition. For all these reasons, the interpretation of the data as support for split attention is subject to significant doubts.

An additional drawback is that the results could also be accommodated by a zooming model of attention (Criterion 4). In a second experiment, Bichot et al. (1999) therefore inserted several distractors in between target locations (including distractors equilinearly placed between target locations). This resulted in a small decrease in performance to about $40 \%$ correct for both simultaneous and sequential conditions (i.e., $80 \%$ correct for individual decisions). Bichot et al. stressed that performance in simultaneous and sequential conditions was again equal and concluded that their experiment supported divided attention. However, the decrease in overall performance compared with that of their first experiment (its statistical significance was not tested) suggests that the distractors had at least some detrimental influence in both the sequential and the simultaneous conditions. This means that attention was either zoomed to include all stimulus locations or serially shifted and that these mechanisms could account for performance in both the sequential and the simultaneous condition. Further, the addition of the distractors in two fixed locations aligned with target locations in this experiment was insufficient to exclude the possibility of an annular or curved elliptical region of attentional enhancement avoiding the distractor locations (Criterion 4). In sum, the experiments from Bichot et al. discussed here do not strongly support the divided attention hypothesis.

Evidence from multiple-object tracking experiments. Pylyshyn and Storm (1988) demonstrated that it is possible to track several moving objects intermingled with other moving distractor objects (see also Cavanagh \& Alvarez, 2005; Flombaum, Scholl, \& Pylyshyn, 2008; Makovski, Vázquez, \& Jiang, 2008; Scholl \& Pylyshyn, 1999). This paradigm is very different from classical divided attention paradigms, but it has been claimed that the ability to simultaneously track several moving objects supports the hypothesis of spatially divided attention (Cavanagh \& Alvarez, 2005). We therefore discuss some of the findings in this interesting field of research and their implications for divided spatial attention.

In the multiple-object tracking paradigm, a number of simple shapes (e.g., small squares) are presented, each moving through space following its own curved pathway (other types of tracking are not considered; see Alvarez \& Cavanagh, 2005). Prior to movement onset, a few of the objects briefly change color (or are briefly flashed on and off) to indicate they are the targets that must be tracked, but during movement there is nothing that distinguishes target from distractor objects. Nevertheless, participants typically are capable of tracking four to five target objects presented among a similar number of distractor objects (visit http://ruccs.rutgers .edu/finstlab/demos.htm for demonstrations of this paradigm).

Original models by Pylyshyn (1989, 2000, 2001, 2003, 2007) of how multiple objects can be tracked simultaneously did not invoke split spatial attention. Pylyshyn proposed that by the brief change of the target objects at the beginning of the task, each of these objects is indexed with a so-called finger of instantiation, which shares similarities with the construction of "object files" (Kahneman \& Treisman, 1984; Kahneman, Treisman, \& Gibbs, 1992; Oksama \& Hyönä, 2004), which contain relevant information about each object and help to select various types of information about the object at once (e.g., previous location, speed, curvature of the motion path). Pylyshyn argued that although spatial attention is required to index the objects in the beginning of the task (i.e., when they change color), spatial attention is not involved in the actual tracking of the objects (see, e.g., Pylyshyn \& Annan, 2006). In agreement with this view, Blaser, Pylyshyn, and Holcombe (2000) showed that superimposed objects (sharing the same location) can be independently tracked when "moving through feature-space" (i.e., when their features change over time). This shows at the very least that there is no close link between hypothetical divided spatial attention and the tracking of multiple objects.

Cavanagh and Alvarez (2005), however, suggested that the tracking of target objects is mediated by fitting each target object with its own focus of spatial attention. Furthermore, Cavanagh and Alvarez found that when four targets were presented, they could be tracked only when they were divided between hemifields, not when all four were presented within a single hemifield. They therefore proposed independent attentional resources for the two hemifields and suggested that this idea was compatible with a model of divided attention in which the number of separate foci available for tracking would be limited by capacity within each hemifield.

However, in contrast to Pylyshyn and Storm's (1988) original study, Alvarez and Cavanagh (2005) and many other tracking studies did not monitor eye movements. This makes an interpretation of the data difficult, as it is doubtful that the (mostly inexperienced) participants would have successfully suppressed eye movements during the presentation of the dynamic displays used in tracking experiments without eye movement control. Consequently, participants may have applied different tracking strategies in different experimental conditions (Fehd \& Seiffert, 2007). In studies testing bilateral advantages during tracking without eye movement control, the participants' attempt to track objects covertly may have been contaminated by complex patterns of eye movements that may have differed between bilateral and unilateral conditions. For example, fixation errors occur more frequently in 
the horizontal direction (Gowen, Abadi, \& Poliakoff, 2005; Gowen, Abadi, Poliakoff, Hansen, \& Miall, 2007), and this asymmetry might contribute to the differential pattern of results in bilateral and unilateral conditions in Alvarez and Cavanagh. The lack of control over participants' tracking strategies without eye monitoring raises the question how robust the finding of a bilateral advantage during multiple-object tracking really is (see, e.g., Shim, Alvarez, \& Jiang, 2008).

Furthermore, an alternative to the hypothesis of divided attention and separate resources in the two hemifields is that participants made use of cross-callosal integration of information in the left and right hemifields. Participants may have used momentary similarities (or dissimilarities) of motion paths in mirror symmetric locations along the vertical meridian, of which cortical representations are connected by cross-callosal fibers, in order to come to a more compressed representation of relevant motion paths. It has been shown that the analysis of stimuli in mirror symmetrical locations to the left and right of the vertical meridian is faster and more efficient (Saarinen \& Levi, 2000; H. R. Wilson \& Wilkinson, 2002). If this mechanism applies to the tracking task, it may explain why tracking is more efficient for bilateral displays than for unilateral displays. This hypothesis is supported by another experiment by Alvarez and Cavanagh (2005) in which the rotation of stationary patterns was tracked. This experiment used mirror symmetrical locations across either the horizontal or the vertical meridian, and showed a strong bilateral advantage in rotation tracking, in agreement with the hypothesized advantage of mirror symmetry across the vertical meridian. An additional alternative explanation is that a single focus of attention was moving more quickly between left and right hemifields than within a hemifield. Although some studies on exogenous cuing suggest that orienting of attention across the vertical meridian is more time-consuming (Hughes \& Zimba, 1987; Rizzolatti et al., 1987), other studies do not confirm this (Henderson \& Macquistan, 1993; Macaluso \& Patria, 2007); and endogenous cuing experiments (Nomura, Katahata, \& Hashiya, 2005) point to an advantage for horizontal attention shifts. This suggests that efficient shifts of attention between hemifields cannot be discarded as a possibility. The general idea of attention switching during tracking was in fact confirmed by Blaser et al. (2000).

Moreover, some other findings constitute support for a serial mechanism during tracking. Alvarez and Franconeri (2007) have shown a linear relationship between the speed required to achieve near-perfect tracking and the logarithm of the number of targets to be tracked. At very slow speeds (on the order $1 \%$ ), up to seven or eight targets could be tracked; at somewhat faster speeds $(5 \%)$, about four targets could be tracked; and at a still relatively slow speed $(15 \%)$, only a single target could be tracked. The importance of low speeds is compatible with the idea that the position of the target dots is only occasionally inspected by a single traveling spotlight of attention. Two mechanisms might contribute to the trade-off between tracking speed and number of targets tracked. First, it is possible that in the retinotopic map where the dots move, the most recent part of the traversed path is actively maintained. This may involve an interaction between attention and lateral connectivity in early visual areas such as middle temporal (similar to what has been shown in V1 by Roelfsema, Lamme, \& Spekreijse, 1998). When the dots move too fast, limits on the lateral extent of horizontal connectivity may lead to a failure in linking the prior and present positions of a target dot during subsequent "inspections" by the focus of attention. Second, it is likely that in addition to a contribution of low-level perceptual mechanisms, working memory mechanisms may limit the number and speed of the different targets that can be tracked.

Several other studies have attempted to gain insight in the attentional mechanisms underlying tracking, with conflicting results (see Flombaum \& Scholl, 2008; Matsubara, Shiori, \& Yaguchi, 2007; Pylyshyn, 2006). It is clear, however, that performance in tracking tasks is determined by a large set of factors, including the number of targets and distractors, speed of movement, packing density of the objects, the extent to which targets or distractors group together by a common feature, and placement of targets in the same or different hemifields. The results and mechanisms underlying tracking performance may be critically dependent on the presence or absence of eye movements during tracking and the specific stimulus conditions chosen. Hence, more research is required to gain insight into the factors that contribute to multipleobject tracking. Because the critical experiments to understand the mechanisms underlying tracking remain to be done, we did not carry out a methodological evaluation of the discussed tracking studies.

\section{No Conclusive Evidence for Divided Attention From Psychophysical Studies}

The psychophysical evidence on the whole does not yield conclusive evidence in favor of divided spatial attention. Most studies show weaknesses on one or more of the criteria to be fulfilled before split attention can be considered plausible. In many studies, the choice of stimuli makes the task insufficiently difficult. In addition, stimuli are often not presented briefly enough to exclude attentional reallocation during divided attention tasks, and this issue is compounded in studies in which the stimuli are easy to detect or discriminate and not masked. Furthermore, the arrangement of target stimuli, probing stimuli, and distractors often does not take into account that the shape of a unitary region of attentional enhancement might show sufficient flexibility to include target locations and exclude irrelevant regions in the display. Finally, a diverse set of issues has been encountered with the cuing strategies employed in a number of studies, which included the use of inappropriate cue-to-target intervals and the use of featurerelated attention to cue attention to particular regions in the visual field.

\section{Electrophysiological Studies}

Several electrophysiological studies in humans have addressed spatial and temporal aspects of divided attention. The studies that addressed the spatial question are discussed first (Eimer, 2000; Heinze et al., 1994; M. M. Müller, Malinowski, Gruber, \& Hillyard, 2003). Then two studies by Woodman and Luck (1999, 2003) are discussed, which addressed temporal aspects of attentional allocation during two-target search.

\section{Using Electrophysiology to Investigate the Spatial Distribution of Attentional Allocation}

Heinze et al. (1994) used evidence from ERPs (event-related potentials) to suggest that attention is zoomed to include relevant 
locations. Two targets and two distractors were centered on a horizontal line $2.9^{\circ}$ above fixation, $6.3^{\circ}$ and $11.9^{\circ}$ to either side of the vertical meridian in each trial. Target positions were verbally indicated prior to a block of trials. Participants performed a matching task on the two target stimuli next to each other in the same hemifield (adjacent condition) or on the targets separated by a single distractor (separated condition). Heinze et al. found behavioral evidence for distractor interference (reaction times increased by $64 \mathrm{~ms}$, and $d^{\prime}$ increased by 0.52 ), as well as ERP evidence (from the P1 positivity) for attentional enhancement of probing stimuli placed in the location of distractor stimuli in between the target stimuli (the probing stimuli were used only for the measurement of ERPs). This finding is compatible with the zoom lens theory of attention but may also simply reflect limitations in spatial resolution of the ERP technique used. Furthermore, the experimental design used by Heinze et al. does not permit drawing strong conclusions about the distribution of attention. The interstimulus interval (250 ms to several seconds) and the lack of control over the duration of stimulus processing (100-ms exposure duration, unmasked) may have allowed prioritizing one target location before trial onset and switching attention to the other target during stimulus exposure (Criteria 2 and 3 ). The evidence that the task was more demanding in the separated than the adjacent conditions was modest (based mostly on reaction times), and accuracy was very high (on the order of $90 \%$ correct), raising doubts whether the task could not be done in parallel without much focusing of attention (Criterion 1). The large symbol sizes $\left(2.5^{\circ} \times 3.2^{\circ}\right)$ combined with the 100-ms exposure duration raise further questions about the difficulty of the task (Criteria 1 and 2). Finally, the lack of a sufficiently detailed measurement of the attention distribution away from the four stimulus locations precludes any conclusion on the presence of unified or divided attention in this study.

In another ERP study, Eimer (2000) suggested the existence "of selective mechanisms that exclude irrelevant stimuli between two simultaneously attended areas" (p. 255). Participants performed a single-target identification task in which they signaled the target letter $N$ ( $25 \%$ of trials) with a button press and ignored a distractor letter $M(75 \%$ of trials) at one of 12 possible locations along three concentric rings of a $1.7^{\circ}, 2.9^{\circ}$, or $4.1^{\circ}$ radius. Letters were $0.8^{\circ} \times$ $0.8^{\circ}$ in size and presented for $100 \mathrm{~ms}$ without masking. Attention was directed to a single annulus (inner, middle, or outer) or two annuli (inner and outer, or inner and middle). The annuli were demarcated by narrow outlines that remained in view throughout the trial. The different conditions were administered in blocks of 240 trials, and the intertrial interval was $1 \mathrm{~s}$.

The behavioral data showed that reaction times and error rates increased as a function of eccentricity for single annuli (reaction times of 529, 579, and $606 \mathrm{~ms}$ and error rates of $2.9 \%, 8.9 \%$, and $13.2 \%$ for inner, middle, and outer annuli, respectively). In the context of attention to two annuli, reaction times were 564, 584, and $611 \mathrm{~ms}$ for stimuli in inner, middle, and outer annuli, respectively, but error rates were not reported. However, because the overall error rate was $10.4 \%$ and the average error rate for the single-annulus condition was $8.3 \%$, the error rate in the two-annuli condition must have been about $12 \%$.

To investigate effects of spatial selectivity in the different attention conditions, Eimer (2000) compared ERPs to nontarget stimuli within attended regions with ERPs to nontarget stimuli located in unattended regions. Eimer reported that there were no attention-related modulations of $\mathrm{N} 1$ and $\mathrm{P} 1$ components in the single-annulus conditions, but there was an enhanced P3 from a number of electrodes (but not occipital ones). Furthermore, ERPs to attended stimuli in the middle ring in the context of attending middle and outer annuli showed a more negative course beyond $200 \mathrm{~ms}$ poststimulus compared with unattended stimuli in the middle ring in the context of attending the inner and the outer annuli. Eimer interpreted this result as "reflecting a subset of spatially selective processes able to exclude irrelevant stimuli located in between attended regions from full attentional processing" (p. 258).

This conclusion, however, should not be interpreted as support for spatially divided attention as we have defined it. The ERP data did not show the N1-P1 effects that could potentially link ERP results to a spatial distribution of enhanced processing in retinotopic areas in the occipital lobe (see, e.g., Di Russo, Martinez, Sereno, Pitzalis, \& Hillyard, 2001; Eimer, 1999). Instead, the results are related to late, higher order processes that are impossible to directly relate to specific spatial distributions of attention in visual cortex or in the visual field.

Furthermore, the study's experimental design makes it difficult to fully test the hypothesis of divided attention. The task was too easy ( $90 \%$ correct) to significantly draw on attentional resources, which is nonoptimal to induce a division of attention (either by shifting or by parallel allocation of attention; Criterion 1). Moreover, given the limited processing requirements, the 100-ms exposure (unmasked) may well have been sufficient to allow a shift in attention during stimulus processing (Criterion 2), and it is unlikely that a shift would have been detected with the ERP technique used. Eimer (2000) used blocking of trials and long intertrial intervals to facilitate setting up a fixed divided attention distribution (indicating that, a priori, divided attention was not seen as a flexible strategy). However, the long intertrial intervals also may have allowed participants to prioritize processing of one annulus and then shift during stimulus presentation to the other annulus (if the target was absent there; Criterion 3). In the same vein, participants also may have prioritized the outer or the inner annulus prior to stimulus onset, rather than select the two simultaneously - and because of the averaging inherent to ERPs, ERP data would not be able to distinguish these two strategies. In addition, the participants were never required to respond to targets in unattended regions, and therefore there was no behavioral evidence to document that attention was indeed absent in annuli that were not supposed to be attended (Criterion 4). Hence, Eimer's results cannot be considered as support for a division of spatial attention.

M. M. Müller et al. (2003) reinvestigated the possibility of dividing attention by using the steady state visually evoked potential (SSVEP), which is a sustained ERP component elicited by a flickering stimulus in the visual field. SSVEPs oscillate at the frequency of the flickering stimulus, and therefore SSVEPs associated with different stimuli can be separated from one another if the stimuli flicker at different frequencies. In this way, the low spatial resolution of the classical ERP technique can be circumvented, and signals from spatially nearby stimuli can be segregated. Previous studies had already shown that directing attention to a flickering stimulus increases the amplitude of the SSVEP (Morgan, Hansen, \& Hillyard, 1996; M. M. Müller \& Hübner, 2002; M. M. Müller, Picton, et al., 1998). This suggested that 
directing attention to one of several stimuli flickering at different rates should be revealed by an increase in the amplitude of the SSVEP coupled with the attended stimulus.

In M. M. Müller et al.'s (2003) experiment, four small flickering squares were presented on the horizontal meridian; two to the left of fixation (at approximately $4^{\circ}$ and $9^{\circ}$ of eccentricity) and two placed symmetrically to the right. Participants were instructed verbally to attend to two of the squares. In each square, a randomized RSVP sequence of symbols was presented at a rate of $181 \mathrm{~ms}$ per display. The participants' task was to attend to two squares and signal the co-occurrence of the same symbols within the squares (a two-target matching task). This was done in adjacent conditions, with two distractors and two targets side by side, or separated conditions, with a distractor separating two targets. Attention directed to target stimuli in the separated condition (one in the left hemifield, the other in the right hemifield) resulted in increased amplitudes of the SSVEPs coupled to the target stimuli compared with the SSVEPs corresponding to distractor stimuli positioned in between the target stimuli (or distractor stimuli next to the target stimuli but not in between). M. M. Müller et al. suggested therefore that attention could be divided between locations in different hemifields (in support of a splitting model).

Despite the beautiful idea of using temporal tagging to overcome limitations in spatial resolution, the suggestion that the data demonstrate split attention should be taken with caution. Performance in the divided attention task averaged $81 \%$ correct, suggesting the task was not exceedingly difficult (Criterion 1). Paradoxically, performance tended to be better for the separated than for the adjacent condition ( $84 \%$ and $79 \%$, respectively). This raises further doubts with regard of the difficulty of the task and therefore also about the idea that in this case the exposure time of $181 \mathrm{~ms}$ (Criterion 2) would have completely prevented a contribution of attention shifts. Furthermore, the increase of the SSVEPs in target compared with distractor locations was significant but not very large. To really exclude attention allocation to the in-between and other distractor locations, SSVEPs should have been used from at least one location far away from the four locations on the horizontal meridian. This extra location would have provided a reference to test whether the SSVEPs in distractor locations still constitute a significant up-regulation of attentional processing. Because this additional control condition was absent, significant attentional modulation at the in-between distractor locations cannot be excluded. In general, there was an insufficiently dense probing of attentional allocation, and it is plausible that an annular region of attentional enhancement was set up (e.g., a zoom lens with low enhancement in its interior to prevent distractor processing) to preferentially process the target locations (Criterion 4). Moreover, as is the case in many studies of divided attention, M. M. Müller et al.'s (2003) study tested divided attention only for targets placed in left and right hemifields. When all stimuli were placed within a single hemifield, Malinowski, Fuchs, and Müller (2007) failed to find a consistent difference between SSVEPs associated with targets and in-between distractors, with some suppression of distractor processing being present in upper but not lower visual field processing.

In addition to the study by Malinowski et al. (2007), some other studies have reported data hinting at the possibility that processing of targets in separate hemifields might facilitate the division of attention and that division of attention within a hemifield is more difficult or impossible (Alvarez \& Cavanagh, 2005; Awh \& Pashler, 2000; Kraft et al., 2004). Although this is an interesting hypothesis that deserves to be followed up, in some of the experiments findings were confounded with differences between upper and lower visual fields, and in all above-cited studies there were plausible alternative explanations for the separate hemifield advantage that did not require the assumption of divided spatial attention.

\section{Using Electrophysiology to Investigate Temporal Stability of Attention Allocation}

Woodman and Luck (1999) asked their participants to perform a search task simultaneously in left and right hemifields. Participants fixated in the middle of a screen filled with outlines of squares, one side of which (left, right, up, or down) showed a small gap. There were 21 black distractor squares spread in the visual field, and in each quadrant a differently colored (red, green, blue, or violet) square was presented, which was the potential target. The participants' task was to identify as quickly as possible the target with a gap in the left rib. Stimulus displays remained in view for $2 \mathrm{~s}$ (response latencies were on the order of $700 \mathrm{~ms}$ ). Before a trial began, participants were informed which two colors were potential targets, and they were also informed that the probability for one particular color to be a target was much higher $(75 \%$ for $\mathrm{T} 75)$ than for the other color (25\% for T25). They were not told how to use that probability information.

If divided attention is a general-purpose mechanism that is readily available and can be sustained without training in a variety of stimulus situations (e.g., Adamo et al., 2008; Cavanagh \& Alvarez, 2005; McMains \& Somers, 2005; N. G. Müller, Bartelt, Donner, Villringer, \& Brandt, 2003) with little or no cost (e.g., McMains \& Somers, 2005; Scharlau, 2004), this mechanism should be very useful in Woodman and Luck's (1999) study. Attention would be allocated in a stable distribution according to target probabilities with more resources for T75 than for T25 (for similar reasoning, see Castiello \& Umiltà, 1992; C. W. Eriksen \& Yeh, 1985; LaBerge \& Brown, 1989; McCormick et al., 1998), and electrophysiological measures during performance should therefore not show shifts of attention. Alternatively, if spatial attention is unitary, it might tend to be allocated first to one target (T75) and then shift to the other (T25) according to target probabilities, and electrophysiological measurements should reveal shifts of attention. That finding would strengthen the argument that in unconstrained conditions (long stimulus exposures), unified (serial) attention is used by default.

The presence or absence of shifts was assessed by measuring the $\mathrm{N} 2$ pc ERP component, which is a negative $(\mathrm{N})$ deflection that reaches its minimum $200-400 \mathrm{~ms}$ after stimulus onset (2) and is recorded posteriorly (p) on the scalp over the hemisphere contralateral (c) to the hemifield to which attention is directed. Modulations in the negativity of this postperceptual component have been shown to be related to attentional selection and to changes in covert attention (Luck \& Hillyard, 1994a, 1994b).

A comparison of the $\mathrm{N} 2 \mathrm{pc}$ recorded from left and right halves of the scalp (with T75 and T25 placed in opposite hemifields) showed that first (200-300 ms) the N2pc contralateral to T75 was more negative than the $\mathrm{N} 2 \mathrm{pc}$ contralateral to $\mathrm{T} 25$, whereas later on (300-400 ms) this became reversed. This result is compatible with 
a reallocation of attentional resources from one location to another and rejects the hypothesis that participants in the used paradigm would set up a sustained, parallel distribution of attention with unequal resources allocated to the two target locations. An elegant study that used a similar experimental design (Woodman \& Luck, 2003) confirmed and strengthened that conclusion.

It is interesting that the electrophysiological data indicate that movements of focused attention can be completed within possibly as fast as $100 \mathrm{~ms}$ (Woodman \& Luck, 1999), to about $150 \mathrm{~ms}$ (Woodman \& Luck, 2003). This implies that stimulus presentations in the order of 150-200 ms used by many studies may be too long to prevent shifts of attention.

The question can be asked why Woodman and Luck (1999, 2003) did not perform their experiment with equal-probability targets (e.g., T50 in each hemifield). The answer resides in the fact that ERP data analysis involves averaging over many trials. A lack of modulation of the averaged N2pc component would not be interpretable: It might indicate attention shifts from left to right, or vice versa, with equal probability on individual trials or no shifts at all. Hence, it could be argued that for the specific condition of equal-probability targets in the left and right hemifields, participants might have employed a divided attention strategy. However, if the hypothesis of a flexible division of spatial attention is to be taken seriously, it is hard to see why it would function only when two stimuli in different locations have the same probability of being a target and not when target probabilities are unequal. The experiments of Woodman and Luck indicate that in absence of time pressure, participants will shift attention rather than maintain a stable distribution of parallel, divided attention. Or, put differently, for experimental designs that would fail all our criteria for a stringent test of divided attention, human observers appear to use serial strategies. Woodman and Luck's studies confirm that observers use a unitary focus by default and that it is reasonable to treat the unitary attention view as the null hypothesis in divided attention studies (see introduction).

\section{No Conclusive Evidence for Divided Attention From Electrophysiology Studies}

Altogether the electrophysiological data suggest that setting up stable distributions of divided attention is not the default way in which humans solve tasks that require monitoring of stimuli in several locations (Woodman \& Luck, 1999, 2003). When attempts are made to constrain stimulus conditions to minimize the possibility of attention shifts, the evidence for divided attention remains weak for interhemispheric division of attention (M. M. Müller et al., 2003) and inconsistent for intrahemispheric division of attention (Malinowski et al., 2007). In sum, the evidence from electrophysiological studies does not provide overwhelming evidence in favor of divided attention.

\section{Functional MRI Studies}

Functional MRI studies have proven to be an effective tool to study attentional enhancement in retinotopic visual areas, which can be demonstrated in the presence (Brefczynski \& DeYoe, 1999; N. G. Müller et al., 2003; Tootell et al., 1998) or the absence of attended stimuli (Kastner et al., 1999). Because of the relatively good spatial resolution of fMRI, a number of fMRI studies have aimed to investigate attentional modulation in between multiple regions of attentionally enhanced processing, the presence of which is instrumental in distinguishing between single-focus and splitting models of attention.

\section{Using fMRI to Investigate the Spatial Distribution of Attentional Allocation}

McMains and Somers (2004) designed an fMRI experiment in which participants simultaneously monitored two of five available RSVP streams of small numbers and letters (one stream in each quadrant at $3.6^{\circ}$ of eccentricity and one in the middle of the screen at fixation; eye fixation was not monitored). The RSVP streams that had to be monitored by the participants were in a lower and an upper quadrant of different hemifields. In the RSVP streams, a new number or letter was presented every $173 \mathrm{~ms}$. Participants had to report when matching numbers were present simultaneously in the two attended RSVP streams (numbers always came in pairs). Based on a region-of-interest approach in retinotopic visual areas, the fMRI data showed attentional modulation for the RSVP streams that participants had to attend, and no attentional modulation for the RSVP stream in between (compared with the other two unattended RSVP streams).

The suggestion that this experiment would conclusively demonstrate divided attention runs into several problems. First, in the scanner, participants carrying out a control task in a single location (in which digits had to be matched with a prespecified target number) showed indistinguishable performance (84\% correct) compared with the divided attention task ( $81 \%$ correct). This indicates the task was not well suited to investigate divided attention (Criterion 1). Outside the scanner, for the 173-ms duration used in the fMRI experiment, psychophysical performance in both the divided attention task and the control task was close to $100 \%$ correct ( $d^{\prime}$ index of 3 ). Even when the presentation duration of the stimuli in the RSVP streams was reduced from $173 \mathrm{~ms}$ to $67 \mathrm{~ms}$, participants reached performance levels of about $70 \%$ correct, in both the divided attention task and the control task. This confirms that the task used during fMRI scanning was easy and that the 173-ms stimulus exposures used during fMRI scanning could not exclude reallocations of attention in participants who chose to do so (Criterion 2). The finding that the fMRI activity associated with the in-between RSVP stream was not modulated may argue against an attentional zoom lens account of the data, but it does not exclude the possibility of shifts of attention. The lack of significant modulation for the in-between RSVP stream may be related to a lack of sensitivity of fMRI for a rapid analogue shift of the focus of attention, or, alternatively, the focus may have been turned off during shifts.

More importantly, the block design of the study makes any interpretation of the data impossible because the temporal resolution limits of the negative blood-oxygen-level-dependent signal (e.g., Amaro \& Barker, 2006; Kim, Richter, \& Uğurbil, 1997) in such design preclude distinguishing activity during intertarget intervals (1.5-2.5 s) from activity during target presentations (173 $\mathrm{ms})$. During the long intertarget intervals, at least two uncontrolled attentional effects might have taken place. First, it cannot be excluded that participants (reflexively) moved attention to one of the locations containing a target after having made the match- 
nonmatch decision. Thus, the enhanced activity for attended RSVP streams may reflect postdecision shifts of attention between attended RSVP stream locations, rather than predecision attentiondriven activity during the $173 \mathrm{~ms}$ of target presentation. Postdecision shifts could have been done without a cost, in light of the long intertarget intervals. Second, participants might have intentionally prioritized processing in one location (knowing that a target in one attended stream implies another one in the other attended stream) to then shift attention to the other location during stimulus presentation (Criterion 3). In any case, it is unlikely that participants would maintain a stable division of attention among locations during the entire 34-s duration of the RSVP presentations. The fMRI signal measured in McMains and Somers's (2004) block design integrated all attention effects at each RSVP location over time, and is uninformative about the dynamical changes in allocation of covert attention. The only conclusion that can be drawn is that over the 34-s stimulation blocks, the total time of attentional enhancement was greater at target RSVP locations than at other locations. Note that the literature on free-viewing eye movements (Bichot et al., 2005; Mazer \& Gallant, 2003; Reinagel \& Zador, 1999; Thompson et al., 2005) and their relationship with covert attention (Mazer \& Gallant, 2003) confirms the idea of preferential orientation of covert attention to objects in a scene rather than empty spaces. To understand the data pattern in McMains and Somers, we assume only that when some of the objects are more relevant than others, they are much more likely to be selected by covert attention (see, e.g., Woodman and Luck, 1999, 2003).

A follow-up fMRI study from McMains and Somers (2005) produced data that are consistent with the reasoning laid out above. McMains and Somers intended to investigate whether there are extra costs associated with the deployment of attention over multiple locations compared with a unitary focus of attention. Participants were presented with five RSVP streams, one in each quadrant and one in the middle. Participants fixated the middle RSVP stream and covertly directed their attention (a) to a single peripheral RSVP stream (see Figure 9A), (b) to two adjacent RSVP streams (Figure 9B), (c) to three adjacent RSVP streams (Figure 9C), (d) to two segregated RSVP streams (Figure 9D), and (e) to an RSVP stream in a location not used in any of the previous four conditions. The fifth condition was used to measure an unattended baseline for the first four conditions. In all five conditions, participants were required to monitor the attended RSVP streams for the appearance of a single target letter (in contrast to the matching task in the previous study).

The participants' letter detection performance was the highest when just one RSVP stream was monitored, lower performance was observed when two RSVP streams were monitored, and worst performance was observed when three RSVP streams were monitored. The magnitude of the fMRI attentional enhancement signal (reflecting a subtraction of attend-ignore conditions) closely mimicked the performance data. The more RSVP regions had to be attended, the lower the attentional enhancement in each region, irrespective of whether those regions were adjacent (for a schematic summary of the design and data, see Figure 9). McMains and Somers (2005) took this as evidence for capacity limitations, with the monitoring of multiple locations taking place at the cost of more limited attentional resources in each attended location. Because no enhancement of the fMRI signal was observed that would correspond to a nonrelevant RSVP stream placed in between two relevant RSVP streams (see Figure 9D), it was concluded that the data were in agreement with a splitting (multiple-foci) model of attention.

As in their preceding study, McMains and Somers (2005) used a task that was not very taxing. Psychophysical performance in the scanner for a single RSVP stream was $87 \%$, which barely dropped (by about 2\%) when two RSVP streams had to be monitored. The authors also mentioned that the effects of increasing the number of RSVP streams was more pronounced in the data set recorded outside the scanner, with performance decreasing from $83 \%$ correct for a single RSVP stream to $75 \%$ for two RSVP streams to $74 \%$ for three RSVP streams. Although not indicated explicitly in McMains and Somers's report, the behavioral data outside the scanner might have been collected prior to scanning, and thus the behavioral data and fMRI signal measured during scanning might have reflected in part some training effects. More importantly, the main shortcoming in McMains and Somers (2004) was also present in their 2005 study. Even if participants maintained a true division of attention during target presentations, it is likely that they moved attention between attended RSVP streams during intertarget intervals ( $3.5 \mathrm{~s}$ long). During these serial reallocations of attention, the probability that a target RSVP stream would be selected by the attentional focus decreases as the number of target RSVP streams in the display increases. This is sufficient to explain the results of McMains and Somers (2005). As in McMains and Somers (2004), the lack of attentional enhancement in between attended RSVP locations may simply reflect lack of sensitivity of the fMRI signal to detect fast shifts of attention, or it may reflect a turning-off of the focus of attention during reallocation.

\section{No Conclusive Evidence for Divided Attention From fMRI Studies}

A fundamental problem of the fMRI studies published to date is that block designs mix activity related to attentional allocation during stimulus presentation with attention during pre- and posttarget discrimination time intervals. Even if reallocations of attention during target presentations had been successfully excluded, reallocations of attention during target-target intervals could still explain the entire pattern of fMRI results. A possibility to avoid this problem may be the use of event-related designs in future fMRI studies. To date, however, fMRI studies have not produced strong evidence that attention can be allocated simultaneously to several noncontiguous locations.

\section{Discussion}

In the last 15 years, a number of studies have been published that claimed support for divided spatial attention. In these studies, participants were instructed to apply the same task (detection, identification, discrimination, or comparison of stimuli) simultaneously in several locations of the visual field (single-task paradigm). From our evaluation of this literature, we conclude that there has been no demonstration so far that attention can be flexibly divided into separate spatial distributions to achieve efficient, simultaneous processing of information in noncontiguous locations. Furthermore, based on theoretical constraints of attentional mechanisms, the single-task paradigms used in current literature may not create the conditions in which a hypothetical 

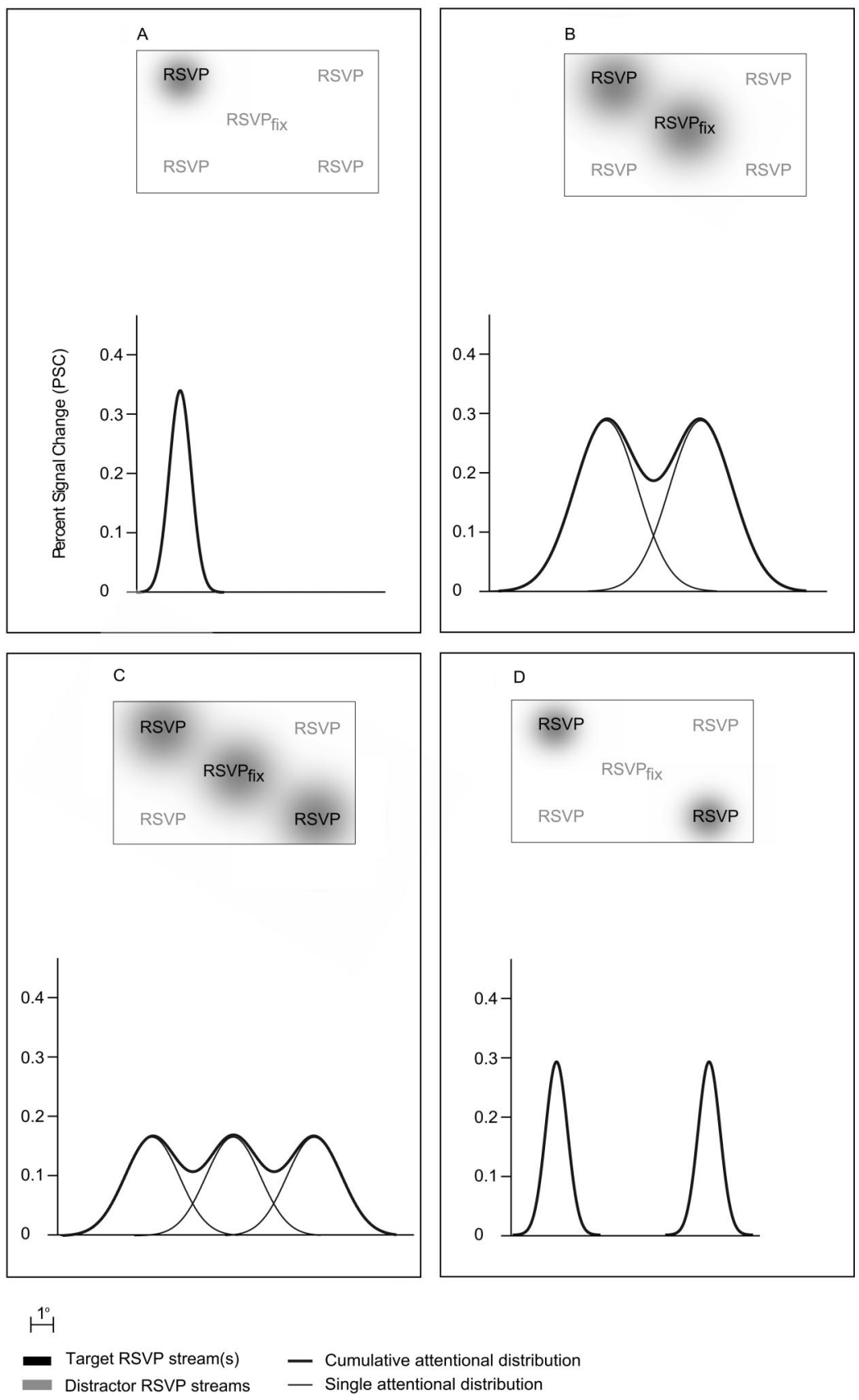

Figure 9. Principal experimental conditions and an appealing but likely incorrect hypothesis about the distribution of attention during each 173-ms presentation of stimuli in target and distractor locations, derived from functional magnetic resonance imaging (fMRI) data in McMains and Somers (2005). Each stimulus display (Figures 9A-9D) contained five rapid serial visual presentation (RSVP) streams, and item size was scaled for cortical magnification (item size not to scale). Attention could be directed to a single-target RSVP stream (A), to two adjacent RSVP streams (B), to three adjacent RSVP streams (C), or to two nonadjacent RSVP streams, separated by a RSVP stream in the middle (at fixation) that had to be ignored (D). The gray ellipsoids in the displays (and cross sections below) show theoretical distributions of attentional enhancement compatible with fMRI (and behavioral) evidence. In the cross sections, thin solid lines show the addends that after summation give the overall attention distribution shown in thick solid lines, in keeping with McMains and Somers' interpretation. Fix = fixation. 
division of spatial attention would permit effective processing of the target stimuli. Instead, dual- or multiple-task paradigms in which very different tasks are applied simultaneously on target stimuli in different locations may provide a better approach to demonstrate a division of spatially divided attention. We argue further that rather than a flexible strategy that can be applied in any stimulus situation, divided attention is a skill that must be acquired through training to process stimuli in unusual task and stimulus conditions, whereas ordinarily unified attention is the default. Taking these points together creates a fundamentally new perspective on the topic of divided spatial attention.

\section{According to Strict Criteria, Divided Spatial Attention Remains to Be Demonstrated}

Typical of current studies of divided attention is that they used a single task that was applied in multiple locations (single-task paradigm). During our analysis of these studies, we have used a restrictive definition of divided attention. Attention is divided when the presence of two or more stable attentional foci can be demonstrated, in the absence of attentional modulation outside those foci, and in the absence of a reallocation of attentional resources from one to the other focus during divided attention trials. This definition has led to the formulation of four criteria that we have used to evaluate studies of divided attention. In Table 1, each cell corresponds to a test of a particular criterion in a specific study. Normal, italicized, or bolded text is used to visually show the outcome of a test. A cell with normal font indicates that a criterion was positively evaluated. A cell with text in italics or bold indicates that a study failed a criterion test, based on one or more data included in that cell (for details, see main text and table note). Text in italics refers to a criterion test that instills at least moderate doubt on the conclusions of the study (i.e., a moderate decline in plausibility), and text in bold refers to a criterion test that instills strong doubt on the conclusions of the study (i.e., at least a strong decline in plausibility). Although some discussion about the outcome of a minority of the tests may be possible, Table 1 reveals important limits on the validity of conclusions from individual studies. Moreover, Table 1 shows that for the field of divided attention as a whole, it seems hard to maintain the idea that the accumulation of evidence from the different studies strengthens the case in support of spatial divided attention.

Table 1 shows that there is not a single study that passes all four criterion tests. All studies fall short against Criterion 4, which requires a sufficiently detailed assessment of the attentional landscape around target locations to exclude that a single continuous attention region of enhanced processing connects the two targets locations. Furthermore, with the exception of Experiment 6 in Bichot et al. (1999), all studies show additional shortcomings on Criteria 1-3, which are designed to test task difficulty, restriction of processing time (stimulus exposure), and length of cue-to-target intervals (or other pretarget periods). In some studies, combinations of easy tasks with stimulus exposures that were too long (and unmasked) make it doubtful that focused attention was necessary to solve the task or that focused attention could not be moved between target locations. In other studies, long cue-to-target intervals were not combined with measures to prevent plausibly successful strategies of prioritizing a single target location prior to stimulus onset. Remarkably, most divided attention studies use unmasked stimuli, and it is well known that even when using short exposures, this is not an effective way of limiting visual processing time. Furthermore, a majority of studies did not monitor eye movements, which raises questions about the effective control of stimulus duration and retinal stimulus position.

The overall pattern of criterion test outcomes in Table 1 shows that single-target detection or identification studies using multiple relevant locations show more weaknesses than two-target identification or matching studies. We also found that ERP and fMRI studies are among the studies showing the weakest experimental design. This may be due in part to compromises in experimental design to accommodate limitations of the techniques used to measure brain activity in humans (e.g., Amaro \& Barker, 2006; Kutas \& Dale, 1997; Luck, 2004; Menon \& Kim, 1999). The systematic evaluation of divided attention studies by using specific design criteria not only shows the weaknesses of current literature as a whole but also provides guidelines for the design of future divided attention studies.

\section{Theoretical Constraints for Spatial Divided Attention}

Many studies of divided attention operate in isolation from neurophysiological findings in the field of selective attention. Here we argue that these findings place constraints on the conditions in which future studies may show divided attention and suggest that a shift from a single-task paradigm to a dual-task paradigm may offer new avenues to investigate this issue. Note that the terms single-task paradigm and dual-task paradigm refer, respectively, to associating the same task to two relevant locations, and associating a different task to each location. This terminology should be distinguished from the terms single-target design and two-target design, which refer, respectively, to the presentation of only a single target at a time in a randomly selected relevant location and the presentation of two targets at once in the relevant locations. We assume that the discussion of dual-task paradigms and two-target designs can be expanded toward multiple-task paradigms and multiple-target designs, respectively.

Constraints placed on divided spatial attention by biased competition. One theoretical framework that places limits on divided attention is standard biased competition theory, in which parallel competition across the visual field is modulated by spatial attention focused on the target selected for action, in the context of a single-task paradigm (Desimone \& Duncan, 1995; see introduction). Competition follows from the anatomical principle that inputs from lower order neurons converge onto higher order neurons, which leads to automatic sensory interaction between stimuli placed in the higher order neurons' RFs. The anatomical feature of converging connectivity leads to increasingly larger RFs at increasingly higher levels of the visual system. Convergence applies to both inhibitory and excitatory inputs, so that the output of a neuron is determined by the mixture of both. Grossberg (1973) derived an important equation that formalizes this principle. In this equation, the spiking rate of a neuron is determined by a maximum spiking rate multiplied by the ratio between excitatory inputs and the sum of excitatory and inhibitory inputs. This equation explains why the response to two stimuli in a neuron's RF is the average of the responses to the individual stimuli rather than a sum. Reynolds et al. (1999) have used the term competition to refer to this averaging process, and they further demonstrated that the effect of attention is to bias the competition in favor of the attended stim- 
ulus in the RF. The term biased competition, therefore, refers to a weighted averaging process, in which the weight of the attended stimulus is increased to permit the propagation of the attended stimulus's information to higher levels in the visual system (and to suppress the irrelevant information). Increasing the weight of the attended stimulus implies that the specific ratio of excitatory and inhibitory inputs that are contributed by the lower order neurons processing the attended stimulus determines the spiking output of the higher order neuron, even though this higher order neuron also contains the unattended stimulus in its RF. Hence, biased competition is a neuronal process that is well suited to explain selective attention. In divided attention tasks, however, participants are typically asked to pay equal attention to two target stimuli, which causes an unweighted averaging process in which the information distinguishing the two stimuli is lost. Thus, divided attention, if it is possible, would in fact be counterproductive.

We speculate that the prohibitive consequences of automatic competition on performance in single-task divided attention paradigms can be avoided. Here we consider single-task paradigms in which stimulus conditions are chosen to exclude serial attention. Under these conditions, a possibility to avoid competition between two stimuli is to ensure that the information related to the stimuli exits the visual system (to drive behavior) before it reaches a level where information of individual stimuli is averaged out (i.e., enters in a competition). This is possible, as most ventral stream areas show direct projections to one or more intraparietal and/or prefrontal visual areas that are related to the programming of motor intention (Distler, Boussaoud, Desimone, \& Ungerleider, 1993; Felleman \& Van Essen, 1991; Ungerleider, Galkin, Desimone, \& Gattass, 2007). This "early exit" strategy is most likely to be successful when using target stimuli that can be processed in early visual areas (V1, V2). For complex target objects that would have to be processed fully (at the highest levels of temporal cortex) in order to be identified, this strategy would not be available, and divided attention might not be possible. Divided attention would not be possible because high-level neurons have RFs of the size of the entire visual field, and divided attention to the objects would therefore unavoidably lead to an unweighted averaging process and loss of information. Accordingly, a potential demonstration of divided attention may have the highest chance of success when using relatively simple stimuli in difficult tasks in which the attentional processing demands reach capacity limitations already at a low level of visual processing. This view fits with models of attention that leave room for some parallel processing of stimuli up to a particular level of processing and postulate capacity limitations for later, full processing of complex objects (Broadbent, 1958; Lachter, Forster, \& Ruthruff, 2004; Lavie, 1995; Lavie \& Tsal, 1994). Note that the constraints provided by standard biased competition apply only to paradigms in which the target stimuli are presented simultaneously in the relevant locations. In single-target paradigms competition is avoided, and therefore the constraints derived from biased competition do not apply. However, the theoretical constraints offered in the next section do apply to both single-target and multiple-target designs.

Constraints placed on divided attention by the "communication through neuronal coherence" theory. A functional principle that is relevant in determining the theoretical limits of divided attention is that populations of neurons in the visual system (and in the brain as a whole) communicate with one another by synchronizing their activity (e.g., Buschman \& Miller, 2007; for detailed reviews, see Buzsaki, 2006; Fries, 2005; Fries, Nikolić, \& Singer, 2007). Fries (2005) has referred to this idea as the "communication through neuronal coherence" hypothesis. This principle provides a mechanistic basis to understand why carrying out a single task on two or more stimuli or locations at the same time may be difficult to achieve.

When two stimuli are present in the visual field and processed at a lower hierarchical level of the visual system, there will be one population of neurons with small RFs containing one of the stimuli and another population of neurons with small RFs containing the other stimulus. Competition is related to the effectiveness of each lower order population in influencing firing rates in a neural population in a higher order visual area with RFs encompassing both stimuli (Reynolds et al., 1999). Selective attention to a target stimulus increases the effectiveness of communication among neural populations in different areas that process the attended stimulus (Buschman \& Miller, 2007; Fries, 2005; Fries et al., 2007; Fries Reynolds, Rorie, \& Desimone, 2001; Fries, Womelsdorf, Oostenveld, \& Desimone, 2008; Womelsdorf et al., 2007). This is accomplished by bringing locally produced oscillatory activity in the gamma range in separate populations into "resonance" with one another (for simplicity, we do not consider other temporal frequency domains here; see Fries, 2005; Fries et al., 2007, 2001). Thus, a biasing signal from an attentional control structure (Buschman \& Miller, 2007; Gregoriou, Gotts, Zhou, \& Desimone, 2009; Saalmann et al., 2007) may get into resonance with oscillatory activity in the population of lower order neurons processing the target (e.g., Fries et al., 2001, 2008; Womelsdorf et al., 2007), which may, in turn, entrain the oscillatory activity of the higher order neurons receiving input from those lower order neurons. As a consequence, the effective communication between lower order neurons processing the target and higher order neurons will be enhanced, whereas lower order neurons processing the irrelevant stimulus will be excluded from this process (Reynolds et al., 1999). However, when it is attempted to simultaneously carry out the same task on two stimuli, a single biasing signal originating from a single attentional control structure must be split and directed at the two lower order neural populations processing the stimuli. We suggest it is unlikely that the same biasing signal can be tuned at the same time to ongoing oscillatory activity in each of the separate lower order neuronal populations encoding the target stimuli, because of local network factors in each population that produce slight differences in local oscillatory activity (Hasenstaub et al., 2005). Hence, the impact of a "split" biasing signal on two lower order populations processing two stimuli will not be identical and after a brief time interval will necessarily yield a winner and a loser. Prior to the emergence of a winner, populations of neurons encoding the two stimuli may sometimes approach synchrony, which may lead to inappropriate binding of the features of the two stimuli (Gray, 1999; Singer, 2004; von der Malsburg, 1994). This reasoning reinforces the idea that a stable division of spatial attention based on splitting a single biasing signal is unlikely. The uncontrollable effects of splitting a single biasing signal also apply to single-target designs. This is because also prior to target stimulus presentation, it can be expected that a single biasing signal cannot be simultaneously tuned to the different temporal properties of local synchrony in the different groups of neurons with RFs covering the relevant locations. Thus, any at- 
Table 1

Attention to Multiple Locations

\begin{tabular}{|c|c|c|c|c|c|c|c|c|c|}
\hline \multirow[b]{2}{*}{ Item } & \multicolumn{9}{|c|}{ Psychophysics } \\
\hline & $\begin{array}{l}\text { C. W. Eriksen } \\
\text { \& Yeh (1985) }\end{array}$ & $\begin{array}{l}\text { Castiello } \\
\text { \& Umiltà } \\
\text { (1992) }\end{array}$ & $\begin{array}{l}\text { McCormick et al. } \\
\text { (1998) }\end{array}$ & Scharlau (2004) & $\begin{array}{l}\text { Gobell et al. } \\
\text { (2004) }\end{array}$ & \multirow[t]{2}{*}{$\begin{array}{c}\text { Kramer \& Hahn } \\
\text { (1995) }\end{array}$} & \multirow[t]{2}{*}{$\begin{array}{c}\text { Hahn \& } \\
\text { Kramer (1998) }\end{array}$} & \multirow[t]{2}{*}{$\begin{array}{l}\text { Bichot et al. } \\
(1999, \text { E6) }\end{array}$} & $\begin{array}{c}\text { Awh \& Pashler } \\
\text { (2000) }\end{array}$ \\
\hline \multicolumn{7}{|c|}{ General information } & & & \\
\hline 1. Method & $\mathrm{P}$ & $\mathrm{P}$ & $\mathrm{P}$ & $\mathrm{P}$ & $\mathrm{P}$ & $\mathrm{P}$ & $\mathrm{P}$ & $\mathrm{P}$ & $\mathrm{P}$ \\
\hline 2. Hypothesis & Shift & Split & Zoom & Split & Split & Split & Split & Split & Split \\
\hline 3. Design & One target & One target & One target & One target & One target & Two target & Two target & Two target & Two target \\
\hline $\begin{array}{l}\text { 4. Special design } \\
\text { feature }\end{array}$ & & & & Prior entry & & Sudden/no onset & $\begin{array}{l}\text { Stimulus } \\
\text { quality }\end{array}$ & & \\
\hline \multicolumn{10}{|c|}{ Criterion 1 (difficulty) } \\
\hline 5. Training? & $\begin{array}{l}4 \text { sessions, } \\
200 \text { trials }\end{array}$ & 200 trials & No & 16 trials & 1,700 trials & 60 trials & 80 trials & No & 120 trials \\
\hline $\begin{array}{l}\text { 6. Single versus } \\
\text { dual tested? }\end{array}$ & No & No & $\begin{array}{l}\text { Yes (but see Item } \\
\text { 19) }\end{array}$ & Yes & No & No & No & No & No \\
\hline $\begin{array}{l}\text { 7. Percent correct } \\
\text { (1), (2); } \\
\text { reaction time } \\
\text { (1), (2) }\end{array}$ & & & 315,334 & No difference & & & & & \\
\hline 8. Target & Letter (S/Y) & Dot & Dot & Ring $^{\#}$ & Disk & Letter & Letter & Letter & Digit (0-9) \\
\hline 9. Distractors & No & No & No & Ring $^{\#}$ & Disks & Letters & Letters & Letters & Letters \\
\hline 10. Task & Identification & Detection & Detection & Judge order & Search & Matching & Matching & Identification & Identification \\
\hline $\begin{array}{l}\text { 11. Target reaction } \\
\text { time }(\mathrm{ms})\end{array}$ & 420 (valid) & 260 & 325 (valid) & & & $550-700(E 1)$ & $635-800(E 1)$ & & \\
\hline $\begin{array}{l}\text { 12. Target } \\
\text { accuracy (\% } \\
\text { correct) }\end{array}$ & 100 & 100 & 100 & & $78-92^{\# \#}$ & $70-95(E 1)$ & 83-94 (E1) & $40^{\#}$ & 88 (E1, $H$ cuing) \\
\hline \multicolumn{10}{|c|}{ Criterion 2 (stimulus duration) } \\
\hline $\begin{array}{l}\text { 13. Fixation } \\
\text { monitored? }\end{array}$ & (No) & (Yes) & (Yes) & (No) & (No) & (No) & (No) & (No) & (No) \\
\hline $\begin{array}{l}\text { 14. Stimulus } \\
\text { duration (ms) }\end{array}$ & Tachistoscopic & 100 & Unlimited & & 150 & 60 & 70 & 60 & 118 \\
\hline 15. Masking? & No & No & No & & No & Yes & Yes & Yes & Yes \\
\hline \multicolumn{10}{|c|}{ Criterion 3 (pretarget interval) } \\
\hline 16. Cue type & Bar & Frame & Frame & Ring mask & Color grating & Frame & Frame & Shape match & Equal sign \\
\hline $\begin{array}{l}\text { 17. Cue to target } \\
(\mathrm{ms})\end{array}$ & 150 & 500 & 515 & -48 to 48 & $\begin{aligned}>350 \text { for best } \\
\text { performance }\end{aligned}$ & 150 & 150 & 105 & 750 \\
\hline $\begin{array}{l}\text { 18. Target to } \\
\text { target }(\mathrm{ms})\end{array}$ & & & & & & & & & \\
\hline $\begin{array}{l}\text { 19. Study-specific } \\
\text { issues related } \\
\text { to cuing }\end{array}$ & $\begin{array}{l}\text { Imbalance } \\
\text { endogenous/ } \\
\text { exogenous } \\
\text { cues }\end{array}$ & & $\begin{array}{l}\text { Very different target } \\
\text { probability in one } \\
\text { versus two } \\
\text { locations }\end{array}$ & $\begin{array}{l}\text { Feature-related } \\
\quad \text { cuing }\end{array}$ & $\begin{array}{l}\text { Feature-related } \\
\quad \text { cuing }\end{array}$ & & & & $\begin{array}{l}\text { Simultaneous } \\
\quad \text { identification of } \\
\text { two distractors }\end{array}$ \\
\hline \multicolumn{10}{|c|}{ Criterion 4 (noncontiguous attentional distribution) } \\
\hline $\begin{array}{l}\text { 20. Stimulus } \\
\text { arrangement }\end{array}$ & Circular & $\begin{array}{l}\text { LR linear } \\
\quad \text { on } \\
\quad \text { fixation }\end{array}$ & $\begin{array}{l}\text { LR linear on } \\
\text { fixation }\end{array}$ & Triangular & $\begin{array}{l}12 \times 12 \text { disk } \\
\text { array }\end{array}$ & $\begin{array}{l}\text { Arc } \\
\quad \text { above/below } \\
\quad \text { fixation }\end{array}$ & $\begin{array}{l}\text { Arc } \\
\quad \text { above/below } \\
\quad \text { fixation }\end{array}$ & Circular & $\begin{array}{l}5 \times 5 \text { letter/digit } \\
\quad \text { array }\end{array}$ \\
\hline $\begin{array}{l}\text { 21. Number of } \\
\text { stimulus } \\
\text { positions }\end{array}$ & $4^{\#}$ & 2 & 4 & 3 & $144^{\#}$ & 4 & 4 & 8 & $4^{\#}$ \\
\hline $\begin{array}{l}\text { 22. Eccentricity } \\
\text { target (degree) }\end{array}$ & 2.5 & \pm 10 & \pm 10 & $\begin{array}{l}5.7 \text { (E1), } 7.5 \\
\quad(\mathrm{E} 2)\end{array}$ & $18 \times 13.5$ & 8.5 & 8.5 & 6.1 & $6.3 \times 6.3$ \\
\hline $\begin{array}{l}\text { 23. Eccentricity } \\
\text { distractor } \\
\text { (degree) }\end{array}$ & 2.5 & & \pm 5 & $\begin{array}{l}5.7 \text { (E1), } 7.5 \\
\quad(\mathrm{E} 2)\end{array}$ & & 8.5 & 8.5 & 6.1 & \\
\hline $\begin{array}{l}\text { 24. Target- } \\
\text { distractor or } \\
\text { target-probe } \\
\text { distance } \\
\text { (degree) }\end{array}$ & 2 & & 5 & $\begin{array}{l}9.6(\mathrm{E} 1), 14.4 \\
(\mathrm{E} 2)\end{array}$ & 1 & 3.3 & 3.3 & 4.7 & 1 \\
\hline $\begin{array}{l}\text { 25. Target-target } \\
\text { distance } \\
\text { (degree) }\end{array}$ & 3.9 & 10 & 10 & $\begin{array}{l}9.6(\mathrm{E} 1), 14.4 \\
\text { (E2) }\end{array}$ & $>1$ & 12.0 & 12.0 & $\geq 4.7$ & 3 \\
\hline
\end{tabular}




\begin{tabular}{|c|c|c|c|c|c|c|c|c|c|}
\hline \multicolumn{5}{|c|}{ Psychophysics } & \multicolumn{3}{|c|}{ Electrophysiology } & \multicolumn{2}{|c|}{ fMRI } \\
\hline $\begin{array}{c}\text { Kraft et al. } \\
(2004)\end{array}$ & $\begin{array}{c}\text { Godijn \& } \\
\text { Theeuwes } \\
(2003)\end{array}$ & $\begin{array}{l}\text { Baldauf et al. } \\
\quad \text { (2006) }\end{array}$ & $\begin{array}{l}\text { Kawahara \& } \\
\text { Yamada } \\
\text { (2006) }\end{array}$ & $\begin{array}{c}\text { Bichot et al. } \\
\text { (1999, E1, } \\
\text { E2) }\end{array}$ & $\begin{array}{c}\text { Heinze et al. } \\
\text { (1994) }\end{array}$ & Eimer (2000) & $\begin{array}{l}\text { M. M. Müller et } \\
\text { al. (2003) }\end{array}$ & $\begin{array}{c}\text { McMains \& } \\
\text { Somers (2004) }\end{array}$ & $\begin{array}{c}\text { McMains \& } \\
\text { Somers (2005) }\end{array}$ \\
\hline $\begin{array}{l}\text { P } \\
\text { Split } \\
\text { Two target } \\
\text { Easy/hard task }\end{array}$ & $\begin{array}{l}\text { P } \\
\text { Split } \\
\text { Two target } \\
\text { Cue by } \\
\quad \text { saccade }\end{array}$ & $\begin{array}{l}\text { P } \\
\text { Split } \\
\text { One target } \\
\text { Cue by } \\
\quad \text { pointing }\end{array}$ & $\begin{array}{l}\text { P } \\
\text { Split } \\
\text { Two target } \\
\text { First 100-ms } \\
\quad \text { blink }\end{array}$ & $\begin{array}{l}\text { P } \\
\text { Split } \\
\text { Two target }\end{array}$ & $\begin{array}{l}\text { ERP (P1) } \\
\text { Zoom } \\
\text { Two target }\end{array}$ & $\begin{array}{l}\text { ERP (N1/P1/P3) } \\
\text { Split } \\
\text { One target }\end{array}$ & $\begin{array}{l}\text { SSVEP } \\
\text { Split } \\
\text { Two target }\end{array}$ & $\begin{array}{l}\text { fMRI } \\
\text { Split } \\
\text { Two target }\end{array}$ & $\begin{array}{l}\text { fMRI } \\
\text { Split } \\
\text { One target } \\
\text { Attend }>2 \\
\quad \text { RSVPs }\end{array}$ \\
\hline$<192$ trials & No & 96 trials & 20 trials & 48 trials & No & 480 trials & No & 1-3 sessions & $\begin{array}{l}\text { Yes } \\
\quad(\text { unspecified })\end{array}$ \\
\hline Yes & No & No & No & No & $\begin{array}{l}\text { Yes } \\
96,89 ; 537 \text {, } \\
\quad 588\end{array}$ & $\begin{array}{l}\text { Yes } \\
92,88 ; 569 \\
\quad 586\end{array}$ & $\begin{array}{l}\text { Yes } \\
79,84 ; 470,457\end{array}$ & $\begin{array}{l}\text { Yes } \\
84,81 \text { inside }\end{array}$ & $\begin{array}{l}\text { Yes } \\
\text { 83, } 75 \text { outside; } \\
\quad 87,85 \text { inside }\end{array}$ \\
\hline Shape & Letter & $\begin{array}{c}\text { E/3 letter/ } \\
\text { digit }\end{array}$ & Letter & Red digits & Symbols ${ }^{\#}$ & Letter $(N / M)$ & Symbols $\#$ & Digit & Letter $(S / K)$ \\
\hline Shapes & Letters & Digits & Digits & Letters & Symbols & No & Symbols ${ }^{\#}$ & Letters & Letters \\
\hline Matching & $\begin{array}{l}\text { Identification } \\
\quad \text { or matching }\end{array}$ & Identification & Identification & $\begin{array}{l}\text { Report } \\
\quad \text { highest }\end{array}$ & Matching & Identification & Matching & Matching & Identification \\
\hline $\begin{array}{c}833 \text { (easy)/935 } \\
\text { (hard) }\end{array}$ & & & & & $537-591$ & $529-606$ & $451-472$ & & \\
\hline $\begin{array}{l}90(\text { easy)/80 } \\
\text { (hard) }\end{array}$ & 77 (E1) & $86(E 1)$ & $40^{\#}$ & $80-85^{\# \#}$ & 89-96 & $87-97$ & $78-84$ & $\begin{array}{l}100 \text { outside, } 83 \\
\quad \text { inside }\end{array}$ & $\begin{array}{l}79 \text { outside, } 86 \\
\text { inside }\end{array}$ \\
\hline
\end{tabular}

\begin{tabular}{|c|c|c|c|c|c|c|c|c|c|}
\hline (Yes) & (Yes) & (Yes) & (No) & (No) & (EOG) & (EOG) & (EOG) & (No) & (No) \\
\hline 100 & $47-82$ & $170-220$ & 100 & 140 & 100 & 100 & 181 & 173 & 173 \\
\hline No & No & Yes & Yes (RSVP) & Yes (RSVP) & No & No & Yes (RSVP) & Yes (RSVP) & Yes (RSVP) \\
\hline $\begin{array}{c}\text { Frames and } \\
\text { central } \\
3,000-7,000\end{array}$ & $\begin{array}{l}\text { Colored disk } \\
\text { and central } \\
35 / 82 / 129\end{array}$ & Central & Block design & Verbal & Block design & Verbal & Verbal & $\begin{array}{l}\text { 6-s visual } \\
\text { instruction } \\
\text { Block design }\end{array}$ & $\begin{array}{l}\text { 6-s visual } \\
\text { instruction } \\
\text { Block design }\end{array}$ \\
\hline & & & $100-700$ & $140-280$ & $\begin{array}{l}250 \mathrm{~ms} \text { to } \\
\text { several } \\
\text { seconds }\end{array}$ & 1,000 & $\geq 905$ & $1,500-2,500$ & 3,500 \\
\hline
\end{tabular}

\begin{tabular}{|c|c|c|c|c|c|c|c|c|c|}
\hline $\begin{array}{l}\text { Linear } \\
\quad \text { oblique }\end{array}$ & Circular & Circular & $\begin{array}{l}\text { Linear LR } \\
\quad \text { on } \\
\text { fixation } \\
\text { (RSVPs) }\end{array}$ & $\begin{array}{l}\text { Linear LR } \\
\text { on } \\
\text { fixation } \\
\text { (RSVPs) }\end{array}$ & $\begin{array}{c}\text { Linear LR } \\
\text { above/ } \\
\text { below } \\
\text { fixation }\end{array}$ & $\begin{array}{c}\text { Circular (3 } \\
\text { rings) }\end{array}$ & $\begin{array}{l}\text { Linear LR on } \\
\text { fixation }\end{array}$ & RSVP array & RSVP array \\
\hline 4 & 4 & 12 & 6 & $\begin{array}{l}2(\text { E1) }-5 \\
(\text { E2 })\end{array}$ & 4 & $36^{\#}$ & 4 & 5 & 5 \\
\hline $3.4,6.1$ & 10.5 & 7.2 & \pm 1.7 & $\begin{array}{l} \pm 2.5(\mathrm{E} 1 \\
\quad \text { and } \mathrm{E} 2)\end{array}$ & $\pm 12.3, \pm 6.9$ & $1.7,2.9,4.1$ & 4 & 3.6 & 3.6 \\
\hline $3.4,6.1$ & 10.5 & 7.2 & $\begin{array}{c}-1.7,-8 \\
.8,1.7\end{array}$ & \pm 1.25 (E2) & $\pm 12.3, \pm 6.9$ & $1.7,2.9,4.1$ & 9 & 3.6 & 3.6 \\
\hline 2.5 & 8.3 & 3.7 & 0.8 & $1.25(\mathrm{E} 2)$ & 5.6 & $>0.9$ & 5 & 3.6 & 3.6 \\
\hline $2.5,5$ & 8.3 & 7.2 & 3.4 & $\begin{array}{c}5 \text { (E1 and } \\
\text { E2) }\end{array}$ & $5.6,18.2$ & $>0.9$ & 5,13 & $3.6,7.2$ & $3.6,7.2$ \\
\hline
\end{tabular}


Table 1 (continued)

\begin{tabular}{|c|c|c|c|c|c|c|c|c|c|}
\hline \multirow[b]{2}{*}{ Item } & \multicolumn{9}{|c|}{ Psychophysics } \\
\hline & $\begin{array}{l}\text { C. W. Eriksen } \\
\text { \& Yeh (1985) }\end{array}$ & $\begin{array}{l}\text { Castiello } \\
\text { \& Umiltà } \\
\text { (1992) }\end{array}$ & $\begin{array}{l}\text { McCormick et al. } \\
\text { (1998) }\end{array}$ & Scharlau (2004) & $\begin{array}{l}\text { Gobell et al. } \\
\text { (2004) }\end{array}$ & $\begin{array}{c}\text { Kramer \& Hahn } \\
\text { (1995) }\end{array}$ & $\begin{array}{c}\text { Hahn \& } \\
\text { Kramer (1998) }\end{array}$ & $\begin{array}{l}\text { Bichot et al. } \\
(1999, \text { E6) }\end{array}$ & $\begin{array}{c}\text { Awh \& Pashler } \\
\text { (2000) }\end{array}$ \\
\hline $\begin{array}{l}\text { 26. Probing } \\
\text { stimuli? }\end{array}$ & Yes (letter) & No & Yes (dot) & No & No & No & No & Yes (letters) & Yes (digits) \\
\hline $\begin{array}{l}\text { 27. Probe perfor- } \\
\text { mance (\% or } \\
\text { ms) }\end{array}$ & $\begin{array}{l}560 \mathrm{~ms} \\
\quad \text { (invalid) }\end{array}$ & & 335 ms (invalid) & & & & & $10 \%$ & $<40 \%$ (invalid) \\
\hline $\begin{array}{l}\text { 28. Distractor } \\
\text { interference? }\end{array}$ & No & No & No & No & Yes (disks) & Yes (letters) & No & No & No \\
\hline $\begin{array}{l}\text { 29. Interference } \\
\text { effect (\% or } \\
\mathrm{ms} \text { ) }\end{array}$ & & & & & $-(0-20) \%^{\#}$ & $\pm \mathbf{5 \%}, \pm \mathbf{3 0} \mathrm{ms}$ & & & \\
\hline $\begin{array}{l}\text { 30. Landscape } \\
\text { complete? }\end{array}$ & No (2) & No $(0)$ & No (2) & No (1) & No $(72)^{\#}$ & No (2) & No (2) & No (6) & No (2) \\
\hline
\end{tabular}

Note. Summary of 75 evaluations on four design criteria in 19 studies. Cells in italics and bold represent criterion tests with an outcome that at least moderately weakened, and strongly weakened plausibility of a conclusion of divided spatial attention, respectively. For Criterion 2 in Scharlau (2004), no test was performed because attention effects were measured by differences in prior entry. Only $19 \%$ of evaluations passed criterion. Criteria were evaluated on a combination of several items ( 26 divided over four criteria). Reaction and stimulus exposure times are given in milliseconds, accuracy in percentages, and eccentricities and distances in visual degrees. Missing or inapplicable parameters are indicated by empty spaces or by "No." Criterion 1 test: A large, significant performance drop from single- to dual-location condition indicates difficulty (e.g., from $90 \%$ to $75 \%$ ). Most studies do not compare single- and dual-location conditions, in which case overall percent correct was used to test the criterion (we show an average or a range). A divided attention task is difficult when performance is midway between chance and perfect performance ( $\leq 75 \%$ correct for $50 \%$ chance level) and when long response latencies ( $>800 \mathrm{~ms}$ ) are observed. Criterion 2 test: Stimulus duration should be $100 \mathrm{~ms}$ or less, and the stimuli should be masked. For rapid serial visual presentations (RSVPs), we accepted stimulus durations up to $200 \mathrm{~ms}$ in difficult tasks. Unmasked stimuli raise moderate to strong doubt depending on stimulus duration and task difficulty (Criterion 1). Criterion 3 test: Brief cue-to-target intervals (150 ms or less) prevent focusing attention on one of the target locations prior to target presentation (and, in two-target studies, subsequent shifting to the second target location during stimulus presentation). Hence, long cue-to-target intervals ( $>200 \mathrm{~ms}$ ) combined with stimulus durations that allow participants to shift attention (>100 ms unmasked; Criterion 2) cause moderate doubt about a study's conclusions. In two-target (or multiple-target) RSVP studies, we assumed an attentional blink of $700 \mathrm{~ms}$ and considered target-target intervals greater than $900 \mathrm{~ms}$ as reason for moderate doubt about attentional allocation prior to target presentation if stimulus exposure durations were too long (see Criterion 2) to prevent rapid attention shifts. Criterion 4 test: Upper bounds of attentional spatial resolution at eccentricities of $1.25^{\circ}, 2.5^{\circ}$, $5^{\circ}$, and $10^{\circ}$ are estimated $0.5^{\circ}, 1^{\circ}, 2^{\circ}$, and $4^{\circ}$, respectively. When spacing between a target and distractor (probing) location (Items 24 and 25 , Criterion 4 ) exceeds the upper bound of attentional resolution, endogenous attention is considered to have sufficient resolution to form a unitary distribution that may include target and avoid distractor (probing) locations, thereby undercutting a conclusion of divided spatial attention. (1) Methods: psychophysics (P), electrophysiology (event-related potential [ERP]; steady state visually evoked potential [SSVEP]), and functional magnetic resonance imaging [fMRI]). (2) Hypotheses: serial reallocation (shift), zooming (zoom), or splitting (split) of spatial attention (null hypotheses for a study). (3) Processing at multiple locations is tested with a single target (one-target design) or multiple targets (typically two-target design). (4) Special design feature is indicated when present. (5) Training prior to experiment is indicated in number of trials and/or sessions. (6) Single- versus dual-location test of difficulty has been performed (yes) or not (no). (7) Performance in single- versus dual-location test is indicated with four numbers indicating accuracy in single- and dual-location test and reaction time in single- and dual-location test, respectively. When only two numbers are shown, two-digit and three-digit values refer to accuracy and reaction times, respectively. (8) Target stimulus is $1^{\circ} \times 1^{\circ}$ or less in size, except for three studies marked by a hash sign: Scharlau (1999), size $=1.9^{\circ} \times 1.9^{\circ}$; Heinze et al. $(1994)$, size $=2.5^{\circ} \times 3.2^{\circ} ;$ and $\mathrm{M}$. M. Müller et al. $(2003)$, size $=2.5^{\circ} \times 3.2^{\circ}$. (9) Same conventions as in Item 8. (10) Operation performed on target stimuli by participants. (11) Target reaction time is indicated as an approximate range in different conditions or as an average. "Valid" refers to validly cued targets. (12) Target accuracy is indicated as an approximate range or average percentage correct. For Bichot et al. (1999, Experiment 6 [E6]), Awh and Pashler (2000), and Kawahara and Yamada (2006), random performance level was lower than 50\% (indicated with hash sign); for Gobell et al. (2004) and Bichot et al. (1999, Experiments 1 and 2 [E1, E2]), we converted performance

tempt to divide spatial attention over relevant locations during the preparatory cue-to-target interval will lead to an unstable division of attention over the relevant locations. From these considerations, it can be derived that divided attention is either counterproductive (i.e., destroying individual stimulus representations) or impossible (i.e., unavoidably leading to an attention shift). Recent findings of Dubois et al. (2009) support this view.

A dual-task approach to study divided attention. Earlier in the Discussion, we suggested an early-exit strategy to permit low-level processing of targets during divided attention. Here we suggest that by applying different tasks to two target stimuli, two control structures may be recruited, each of which may impose its own biasing signal, so that the two relevant stimuli are processed by distributed ensembles of neurons synchronized in different (gamma) frequency (sub)ranges. This idea is related to the suggestion that multiple objects might be attended by assigning different phase slots to them even within the same frequency band (Duncan, 1996). In this theoretical perspective, the need to limit processing depth to achieve divided attention may be less stringent in dual-task than in single-task paradigms.

In contrast to an influential series of publication from Goldberg and colleagues (e.g., Bisly \& Goldberg, 2003; Colby \& Goldberg, 1999; Gottlieb et al., 1998) that defends the idea of a single saliency map (lateral intraparietal area) in which "general purpose" relevance would be encoded, other studies have suggested that there may be different saliency maps that encode relevance in the context of different task requirements. Andersen et al. (1997), for example, emphasized that, in most cases, the activity in the lateral intraparietal area is predictive of eye movements and the activity in the parietal reach area is predictive of reaching movements (Quiroga, Snyder, Batista, Cui, \& Andersen, 2006). Thus, lateral intraparietal and parietal reach would be two maps in which the salience of a stimulus in a particular location of the visual field becomes associated with the intention to move different effectors (for related views, see Allport, 1987; Goodale \& Milner, 1992).

If one assumes that there are different saliency maps associated with different effectors (Quiroga et al., 2006; Thompson et al., 2005), a possible way to demonstrate split attention might be to use two effectors: one effector (e.g., the eye) to acquire a target in one location and another effector (e.g., arm and hand) to acquire another target in a different location. In this dual-task paradigm, human observers may be trained to perform a simultaneous reach and eye movement in response to stimuli in different target locations. A study in monkeys (L. A. Snyder, Batista, \& Andersen, 1997) has used a similar paradigm while recording in monkey parietal reach but without testing divided attention. By using two effectors (or using two "sources" for the biasing signal), populations of neurons processing the stimuli might be segregated from one another by adopting different rhythms 


\begin{tabular}{|c|c|c|c|c|c|c|c|c|c|}
\hline \multicolumn{5}{|c|}{ Psychophysics } & \multicolumn{3}{|c|}{ Electrophysiology } & \multicolumn{2}{|c|}{ fMRI } \\
\hline $\begin{array}{l}\text { Kraft et al. } \\
\text { (2004) }\end{array}$ & $\begin{array}{l}\text { Godijn \& } \\
\text { Theeuwes } \\
\text { (2003) }\end{array}$ & $\begin{array}{l}\text { Baldauf et al. } \\
\text { (2006) }\end{array}$ & $\begin{array}{l}\text { Kawahara \& } \\
\text { Yamada } \\
\text { (2006) }\end{array}$ & $\begin{array}{c}\text { Bichot et al. } \\
\text { (1999, E1, } \\
\text { E2) }\end{array}$ & $\begin{array}{l}\text { Heinze et al. } \\
\text { (1994) }\end{array}$ & Eimer (2000) & $\begin{array}{l}\text { M. M. Müller et } \\
\text { al. (2003) }\end{array}$ & $\begin{array}{c}\text { McMains \& } \\
\text { Somers (2004) }\end{array}$ & $\begin{array}{l}\text { McMains \& } \\
\text { Somers (2005) }\end{array}$ \\
\hline \multirow[t]{2}{*}{ No } & Yes (letters) & Yes (digits) & Yes (letters) & No & $\begin{array}{l}\text { Yes } \\
\text { (rectangle) }\end{array}$ & Yes & No & No & No \\
\hline & $58 \%(\mathrm{E} 1)$ & $54 \%($ E1) & $20 \%$ & & $\begin{array}{l}\text { Only for } \\
\text { ERP }\end{array}$ & Only for ERP & & & \\
\hline Yes (shapes) & No & No & No & No & Yes (symbols) & No & Yes (symbols) & Yes (letters) & Yes (letters) \\
\hline $\begin{array}{l}-(0-8) \%, \\
\quad+(40-80) \\
\quad \text { ms }\end{array}$ & & & & & $-8 \%, 54 \mathrm{~ms}$ & & $5 \%,-13 \mathrm{~ms}$ & $-3 \%$ inside & $-2 \%$ inside \\
\hline No $(2)$ & No (2) & No (6) & No (4) & $\begin{array}{c}(0[\mathrm{E} 1]-3 \\
[\mathrm{E} 2])\end{array}$ & No (2) & No $(12)^{\#}$ & No (2) & No (3) & No (2-3) \\
\hline
\end{tabular}

to estimates that would be found if random level had been $50 \%$ (indicated with double hash sign). Other conventions as in Items 7 and 11. (13) Fixation monitoring was carried out with conventional equipment (yes), with electro-oculography (EOG), or not (no). The presence or absence of fixation monitoring is not used for criterion evaluation purposes and is shown solely for completeness (hence placed in parentheses and always in normal font). (14) Stimulus duration is known in all studies but one (C. W. Eriksen \& Yeh, 1985). (15) Masking has been carried out (yes) or not (no). (16) A central, symbolic cue at fixation (indicated as "central"); a cue at the target location (a small bar, frame, grating, shape, colored shape, or other symbols); or a verbal instruction. (17) Interval between cue and target. When a "block design" is used, the relevant variable is the target-to-target interval (Item 18). (18) Cell is left blank when cue-to-target interval (Item 17) is the relevant value for that study. (19) Study-specific issues related to cuing are present in some studies. (20) Stimulus arrangements include "circular" (with a number of stimuli arranged equieccentrically around fixation), "LR linear on fixation" (with stimuli placed on a straight horizontal line to the left and right of fixation), an "array" of stimuli or RSVPs in a gridlike pattern, an "arc above/below fixation" (with stimuli on an arc above or below fixation), and "LR linear above/below fixation" (with stimuli place on a straight horizontal line to the left and right of vertical meridian, placed above or below fixation). (21) Total number of stimulus positions for visual targets and for the distractors/probes used for testing attention allocation away from target positions. For numbers marked with hash sign, see text for details. (22) Target eccentricity is an unsigned number for circular displays or a signed number for stimuli in a linear arrangement, with " \pm "indicating that stimuli are present to the right and the left of fixation at the indicated number of visual degrees. More than one number indicates that more eccentricities for target locations were used. Search arrays are centered on fixation and described by their overall size. Note that these conventions are also used in Items 23-27. (23) Distractor eccentricity; see Item 22. (24) Refers to the smallest distance between a target and a neighboring distractor or probing stimuli used in the study. In Gobell et al. (2000) and Eimer (2000), these distances are variable, and their maximum is limited by display size (only an underlimit is shown in the table). (25) The distance between two target locations. If two values are given, this refers to values from different experiments in the article under study or to the two typical target-target distances in that study. In Gobell et al. (2000) and Eimer (2000), target-target distances are variable, and their maximum is limited by display size (only an underlimit is shown in the table). (26) Probing stimuli are indicated to be present (yes) when targets are occasionally placed in an unexpected position (labeled "invalid probes"; one-target design) or when participants were asked to occasionally report on a distractor (two-target design). The type of probe is indicated in parentheses. (27) Probe performance is indicated by accuracy or reaction time. In studies with a classical invalid cuing design, probe performance is qualified with "invalid." In some electrophysiological studies, probe stimuli are used to measure ERPs, without measuring behavior (indicated as "only for ERP"). (28) Distractor interference is marked as "yes" if it has been measured, with the type of distractor stimulus indicated in parentheses, or marked "no" if not measured. (29) Interference effect (i.e., change in target-related performance due to distractor presence) is shown as decreased (-) or increased (+) accuracy or reaction times. Other conventions as in Items 11 and 12. (30) Refers to whether the attentional distribution has been sampled finely enough to reasonably exclude the possibility that enhanced performance at target locations resulted from a single region of attentional enhancement. Evaluation is based on a comparison of the eccentricity of stimuli (Items 22, 23) and the spacing among stimuli (Items 24, 25) with estimated resolution of spatial attention, on overall stimulus arrangement and target positions (Item 20), and on numbers of probing/distractor locations used to test attention away from target positions in divided attention conditions (numbers in parentheses). For numbers with hash sign, see text for details.

of coherence, and under these conditions, high-level, simultaneous processing of objects in different locations might be possible in divided attention tasks. Different cognitive tasks relying on differential contributions of parietal and prefrontal cortex are associated with neuronal coherence in different frequency ranges (Buschman \& Miller, 2007), and therefore segregated processing of stimuli in different target locations may also be achieved by associating them with different cognitive tasks. In addition, recent neurophysiological findings and modeling studies support the theoretical possibility of simultaneous attention to and representation of multiple objects by differentiating the phase of coherent activity associated with each object (e.g., Günay \& Maida, 2003; Neitzel, Mandon, \& Kreiter, 2009), which may be another neural mechanism that could potentially underlie divided attention to targets associated with different tasks or effectors.

There is a long tradition in using dual-task paradigms to study divided attention (e.g., Pashler \& Johnston, 1998; Ruthruff, Pashler, \& Hazeltine, 2003). However, here we use the term dual-task paradigm in a mechanistic manner that is different from the traditional meaning. Traditional dual-task paradigms usually involve tasks in which participants have to respond with a set of buttons or fingers to one set of stimuli (e.g., letter presentations) and with another set of buttons or fingers to another set of stimuli (e.g., tones). The reaction times of motor responses are used to address issues related to the sequential or parallel allocation of resources to the two tasks, interference, and capacity limitations. In the dual-task paradigm we propose, the aim is to choose two tasks that depend on attentional control structures that use attentional biasing signals in different temporal frequency ranges (e.g., different subdomains of gamma), with the goal of testing the hypothesis of spatially divided attention, which has not been the focus of interest in prior dual-task research.

In summary, we suggest two principal constraints on divided spatial attention and associated sets of predictions. First, with respect to the single-task paradigms that are currently used in the literature and involve dividing attention among simultaneously presented targets in the context of a single task, we suggest that divided attention, if it exists, will be most likely demonstrated with relatively simple stimuli (shapes or patterns) that differ little on one or maybe a few elementary features. We predict that the simultaneous identification or discrimination of multiple highlevel objects (e.g., faces, cars) during hypothetical, truly divided attention will not be possible. The underlying theoretical constraint is that the simultaneous processing of targets receiving the same biasing signal will erase target presentations at the processing level where RFs encompass the target stimuli. Therefore, the only possible strategy for achieving a semblance of divided attention would be to avoid high-level processing. Theoretical considerations on the role of coherence during attention reinforce this 
constraint in multiple- and single-target designs. Second, in a two-task paradigm in which different targets are processed in the context of different tasks, we suggest that processing of the targets at a high level may be achievable within spatially distinct distributions of attention. The theoretical basis for this proposal is that different control structures may create (gamma) coherence in temporal frequency ranges that will be distinct for the subpopulations of visual neurons in retinotopic maps and in high-level visual areas that encode the target stimuli in different locations.

\section{Divided Spatial Attention Is a Skill, Not a Flexible Strategy of Attentional Allocation}

Participants in divided attention studies typically received no significant training prior to experimentation (see Table 1). Only five studies indicate that participants received significant training, and in only three of those the number of trials was specified (Eimer, 2000; C. W. Eriksen \& Yeh, 1985; Gobell et al., 2004). McMains and Somers $(2004,2005)$ also reported that their participants received training but did not specify the number of trials. In the remaining studies summarized in Table 1, the number of practice trials given to participants varied between 0 and 200 (59 trials on average). This shows that most investigators implicitly assume that divided attention is an attentional strategy that human observers can apply at will. We suggest that this assumption is incorrect and has led investigators to devise tasks that were easy enough for inexperienced participants to carry out but also unsuitable to demonstrate divided spatial attention.

The idea that a potential demonstration of divided attention will require highly restrictive stimulus conditions in a single-task paradigm, or combined execution of two very different tasks, implies that an experiment designed to test divided attention would necessarily be demanding for the participants. To achieve successful performance in such an experiment, it is likely that much more training will be required than has been applied in divided attention studies to date. Hence, we suggest that future research will show that divided attention is a skill that needs to be trained much like any other perceptual or motor skill. That attention can be trained (e.g., in conjunction search tasks) is supported by several studies (Pavlovskaya, Ring, Groswasser, Keren, \& Hochstei, 2001; Scialfa, Jenkins, Hamaluk, \& Skaloud, 2000), and it is known that plasticity in parietal cortex contributes to these training effects (Walsh, Ellison, Ashbridge, \& Cowey, 1999). Performance of visual skills can be highly specific to the precise stimuli and stimulus locations used during training. If future research confirms that the ability to divide attention develops over time with practice, an important question will be whether the acquired skill is specific to trained locations, stimuli, task, and cuing strategies (see, e.g., Karni \& Bertini, 1997).

\section{Sequential Allocation of Spatial Attention Is a Default Strategy, Whereas Divided Spatial Attention Is Not}

Some modeling studies (e.g., Bundesen, Habekost, \& Kyllingsbæk, 2005; Standage, Trappenberg, \& Klein, 2005a, 2005b) have devised network architectures that produced simulated output compatible with a division of spatial attention over noncontiguous locations. The massive parallelism in the design of the visual system (Bender, 1981; Lyon, Jain, \& Kaas, 2003; McIlwain, 1973;
Stanton, Bruce, \& Goldberg, 2004; see also Andersen \& Buneo, 2002; Colby \& Goldberg, 1999) seems in line with the idea of a parallel modulation of retinotopic bottom-up sensory input by top-down biasing signals (see also LaBerge \& Brown, 1986, 1989; Pratt \& Quilty, 2002). However, the presence of parallel, topographically organized connectivity says little about other structural and functional constraints in the visual system that may determine whether spatial attention can be split. In the introduction, we proposed several theoretical arguments suggesting that human observers by default use unified (serial) attention strategies whenever stimulus conditions permit. Here we present additional empirical backing for this view. Rollenhagen and Olson (2005) found that monkeys confronted with two stimuli in their visual field while maintaining central fixation showed modulations in the response of inferior temporal neurons in the order of 5-6 Hz. This slow oscillatory activity was present in the context of an attention task (in which one stimulus was a target and the other a distractor) and to a lesser degree during passive fixation. Although Rollenhagen and Olson did not discuss this possibility, a plausible explanation for their findings is a covert shifting of attention between the stimuli. As in monkey studies of parietal function during attention (Balan \& Gottlieb, 2006; Colby \& Goldberg, 1999; Gottlieb et al., 1998), the monkeys were not required to perform a divided attention task on the stimuli, and there was no attempt to impose a true division of attention. The data, therefore, only suggest that the default mode to inspect multiple stimuli is by shifting attention, not by allocating attention in parallel. A recent psychophysical study of divided attention in humans came to a very similar conclusion. VanRullen, Carlson, and Cavanagh (2007) used a difficult target detection task to determine a psychometric function relating detection probability to target presentation time. This function was then used to derive quantitative predictions of target detection probability as a function of presentation time from parallel and serial computational models during detection in two locations. They found that behaviorally measured probabilities during dual-task requirements were in line with a serial model, and they found optimal fits with their data when assuming that the focus of attention discretely sampled the environment at $7 \mathrm{~Hz}$ (shifting every $143 \mathrm{~ms}$ ), close to the 5-6- $\mathrm{Hz}$ modulations in spike rate observed in monkey inferior temporal neurons (Rollenhagen \& Olson, 2005). VanRullen et al. also pointed out that the increase in target detection probability for a single target as a function of presentation time was in agreement with the same $7-\mathrm{Hz}$ sampling rate. They suggested that this cyclical process of attentional allocation is not unlike the discrete way in which sensory information itself is gathered: during eye fixations in vision, in many species during whisking in the somatosensory systems, or during sniffing in the olfactory system.

Moreover, data from a large set of multiple-target search studies analyzed by Thornton and Gilden (2007) in a manner that can be argued to be more decisive than in prior studies (reviewed in Townsend, 1990, and in Townsend \& Wenger, 2004) suggests that there is an identifiable category of search tasks in which humans use a single, serially allocated focus of attention. In a related field of research, Roelfsema, Khayat, and Spekreijse (2003) have recently shown that also the subtasks (related to the concept of visual "routines"; Ullman, 1984) of more complex attentional tasks (such as the combined visual search and spatial curve tracing task used in their study) are performed in serial order rather than in parallel 
(see also Houtkamp \& Roelfsema, 2009). The strong evidence for serial processing of multiple-target stimuli or tasks in a variety of conditions underscores the validity of taking the unified attention hypothesis as the null hypothesis when evaluating or designing studies of divided spatial attention.

\section{Conclusion}

In summary, we suggest that up to today, there are no studies that have convincingly demonstrated divided spatial attention. The current absence of that demonstration evidence does not exclude the possibility that with better designed future experiments, divided spatial attention can be demonstrated. We hypothesize that a demonstration of efficient, high-level processing of several objects by means of split spatial attention requires the use of specific stimulus conditions and tasks that cannot be carried out without considerable training. A compelling demonstration of divided attention in humans may come from a combination of a clever psychophysical design with electrophysiological methods to demonstrate a lack of attentional movement and fMRI to demonstrate a lack of attentional modulation outside attended locations. The most definite evidence for divided spatial attention might come from neurophysiological recording studies in nonhuman primates because of the superior spatial and temporal resolution of the signal that can be recorded in these experiments.

\section{References}

Adamo, M., Pun, C., Pratt, J., \& Ferber, S. (2008). Your divided attention, please! The maintenance of multiple attentional control sets over distinct regions in space. Cognition, 107, 295-303.

Allport, D. A. (1987). Selection for action: Some behavioral and neurophysiological considerations of attention and action. In H. Heuer \& F. Sanders (Eds.), Perspectives on perception and action (pp. 395-419). Hillsdale, NJ: Erlbaum.

Alvarez, G. A., \& Cavanagh, P. (2005). Independent resources for attentional tracking in the left and right visual hemifields. Psychological Science, 16, 637-643.

Alvarez, G. A., \& Franconeri, S. L. (2007). How many objects can you attentively track?: Evidence for a resource-limited tracking mechanism. Journal of Vision, 7, 1-10.

Amaro, E., Jr., \& Barker, G. J. (2006). Study design in fMRI: Basic principles. Brain and Cognition, 60, 220-232.

Andersen, R. A., \& Buneo, C. A. (2002). Intentional maps in posterior parietal cortex. Annual Review of Neuroscience, 25, 189-220.

Andersen, R. A., Snyder, L. H., Bradley, D. C., \& Xing, J. (1997). Multimodal representation of space in the posterior parietal cortex and its use in planning movements. Annual Review of Neuroscience, 20, 303-330.

Arguin, M., Lassonde, M., Quattrini, A., Del Pesce, M., Foschi, N., \& Papo, I. (2000). Divided visuo-spatial attention systems with total and anterior callosotomy. Neuropsychologia, 38, 283-291.

Atkinson, R. C., Holmgren, J. E., \& Juola, J. F. (1969). Processing time as influenced by the number of elements in a visual display. Perception \& Psychophysics, 6, 321-326.

Averbach, E., \& Coriell, A. S. (1961). Short-term memory in vision. Bell System Technical Journal, 40, 309-328.

Awh, E., \& Pashler, H. (2000). Evidence for split attentional foci. Journal of Experimental Psychology: Human Perception and Performance, 26, $834-846$.

Bacon, W. F., \& Egeth, H. E. (1994). Overriding stimulus-driven attentional capture. Perception \& Psychophysics, 55, 485-496.
Bahcall, D. O., \& Kowler, E. (1999). Attentional interference at small spatial separations. Vision Research, 39, 71-86.

Balan, P. F., \& Gottlieb, J. (2006). Integration of exogenous input into a dynamic salience map revealed by perturbing attention. Journal of Neuroscience, 26, 9239-9924.

Baldauf, D., Wolf, M., \& Deubel, H. (2006). Deployment of visual attention before sequences of goal-directed hand movements. Vision Research, 46, 4355-4374.

Banich, M. T. (1998). The missing link: The role of interhemispheric interaction in attentional processing. Brain and Cognition, 36, 128-157.

Bell, A. H., \& Munoz, D. P. (2008). Activity in the superior colliculus reflects dynamic interactions between voluntary and involuntary influences on orienting behaviour. European Journal of Neuroscience, 8, 1654-1660.

Belopolsky, A. V., Zwaan, L., Theeuwes, J., \& Kramer, A. F. (2007). The size of an attentional window modulates attentional capture by color singletons. Psychonomic Bulletin \& Review, 14, 934-938.

Bender, D. B. (1981). Retinotopic organization of macaque pulvinar. Journal of Neurophysiology, 46, 672-693.

Berger, A., Henik, A., \& Rafal, R. (2005). Competition between endogenous and exogenous orienting of visual attention. Journal of Experimental Psychology: General, 134, 207-221.

Bichot, N. P., Cave, K. R., \& Pashler, H. (1999). Visual selection mediated by location: Feature-based selection of noncontiguous locations. Perception \& Psychophysics, 61, 403-423.

Bichot, N. P., Rossi, A. F., \& Desimone, R. (2005). Parallel and serial neural mechanisms for visual search in macaque area V4. Science, 308, $529-534$.

Bisley, J. W., \& Goldberg, M. E. (2003). Neuronal activity in the lateral intraparietal area and spatial attention. Science, 299, 81-86.

Blaser, E., Pylyshyn, Z. W., \& Holcombe, A. O. (2000). Tracking an object through feature-space. Nature, 408, 196-199.

Boehnke. S. E., \& Munoz, D. P. (2008). On the importance of the transient visual response in the superior colliculus. Current Opinion in Neurobiology, 18, 544-551.

Boynton, G. M., Ciaramitaro, V. M., \& Arman, A. C. (2006). Effects of feature-based attention on the motion aftereffect at remote locations. Vision Research, 46, 2968-2976.

Braun, J., \& Sagi, D. (1991). Texture-based tasks are little affected by second tasks requiring peripheral or central attentive fixation. Perception, 20, 483-500.

Brefczynski, J. A., \& DeYoe, E. A. (1999). A physiological correlate of the "spotlight" of visual attention. Nature Neuroscience, 2, 370-374.

Breitmeyer, B. G., \& Kersey, M. (1981). Backward masking by pattern stimulus offset. Journal of Experimental Psychology: Human Perception and Performance, 7, 972-977.

Bricolo, E., Gianesini, T., Fanini, A., Bundesen, C., \& Chelazzi, L. (2002). Serial attention mechanisms in visual search: A direct behavioral demonstration. Journal of Cognitive Neuroscience, 14, 980-993.

Broadbent, D. E. (1958). Perception and communication. London, England: Pergamon.

Broadbent, D. E. (1982). Task combination and selective intake of information. Acta Psychologica, 50, 253-290.

Brown, W. S., \& Jeeves, M. A. (1993). Bilateral visual field processing and evoked potential interhemispheric transmission time. Neuropsychologia, 31, 1267-1281.

Bundesen, C., Habekost, T., \& Kyllingsbæk, S. (2005). A neural theory of visual attention: Bridging cognition and neurophysiology. Psychological Review, 112, 291-328.

Buschman, T. J., \& Miller, E. K. (2007). Top-down versus bottom-up control of attention in the prefrontal and posterior parietal cortices. Science, 315, 1860-1862.

Busse, L., Katzner, S., \& Treue, S. (2008). Temporal dynamics of neuronal modulation during exogenous and endogenous shifts of visual attention 
in macaque area MT. Proceedings of the National Academy of Sciences, USA, 105, 16380-16385.

Buzsaki, G. (2006). Rhythms of the brain. New York, NY: Oxford University Press.

Caputo, G., \& Guerra, S. (1998). Attentional selection by distractor suppression. Vision Research, 3, 669-689.

Castiello, U., \& Umiltà, C. (1992). Splitting focal attention. Journal of Experimental Psychology: Human Perception and Performance, 18, 837-848.

Cavanagh, P., \& Alvarez, G. A. (2005). Tracking multiple targets with multifocal attention. Trends in Cognitive Sciences, 9, 349-354.

Cave, K. R., \& Bichot, N. P. (1999). Visuospatial attention: Beyond a spotlight model. Psychonomic Bulletin \& Review, 6, 204-223.

Cave, K. R., \& Zimmerman, J. M. (1997). Flexibility in spatial attention before and after practice. Psychological Science, 8, 399-403.

Cepeda, N. J., Cave, K. R., Bichot, N. P., \& Kim, M.-S. (1998). Spatial selection via feature-driven inhibition of distractor locations. Perception \& Psychophysics, 60, 727-746.

Chastain, G. (1992). Time-course of sensitivity changes as attention shifts to an unpredictable location. Journal of General Psychology, 119, 105-111.

Chelazzi, L., Miller, E. K., Duncan, J., \& Desimone, R. (1993). A neural basis for visual search in inferior temporal cortex. Nature, 363, 345-347.

Chirimuuta, M., Burr, D., \& Morrone, M. C. (2007). The role of perceptual learning on modality-specific visual attentional effects. Vision Research, $47,60-70$

Chun, M. M., \& Potter, M. C. (1995). A two-stage model for multiple target detection in rapid serial visual presentation. Journal of Experimental Psychology: Human Perception and Performance, 21, 109-127.

Colby, C. L., \& Goldberg, M. E. (1999). Space and attention in parietal cortex. Annual Review of Neuroscience, 22, 319-349.

Coltheart, M., \& Arthur, B. (1972). Evidence for an integration theory of visual masking. Quarterly Journal of Experimental Psychology, 24, 262-269.

Corbetta, M. (1998). Frontoparietal cortical networks for directing attention and the eye to visual locations: Identical, independent, or overlapping neural systems? Proceedings of the National Academy of Sciences, USA, 95, 831-838.

Corbetta, M., \& Shulman, G. L. (2002). Control of goal-directed and stimulus-driven attention in the brain. Nature Reviews Neuroscience, 3 , 201-215.

Cousineau, D., Charbonneau, D., \& Jolicœur, P. (2006). Parameterizing the attentional blink effect. Canadian Journal of Experimental Psychology, 60, 175-189.

Czerwinski, M., Lightfoot, N., \& Shiffrin, R. M. (1992). Automatization and training in visual search. American Journal of Psychology, 105, 271-315.

Davis, G., Driver, J., Pavani, F., \& Shepherd, A. (2000). Reappraising the apparent costs of attending to two separate visual objects. Vision Research, 40, 1323-1332.

Deco, G., Pollatos, O., \& Zihl, J. (2002). The time course of selective visual attention: Theory and experiments. Vision Research, 42, 2925-2945.

Deco, G., \& Zihl, J. (2004). A biased competition based neurodynamical model of visual neglect. Medical Engineering \& Physics, 26, 733-743.

Desimone, R., Albright, T. D., Gross, C. G., \& Bruce, C. (1984). Stimulusselective properties of inferior temporal neurons in the macaque. Journal of Neuroscience, 4, 2051-2062.

Desimone, R., \& Duncan, J. (1995). Neural mechanisms of selective visual attention. Annual Review of Neuroscience, 18, 193-222.

Desimone, R., \& Ungerleider, L. G. (1989). Neural mechanisms of visual processing in monkeys. In F. Boller \& J. Grafman (Eds.), Handbook of neurophysiology (Vol. 2, pp. 267-299). Amsterdam, the Netherlands: Elsevier.

Desimone, R., Wessinger, M., Thomas, L., \& Schneider, W. (1990). Attentional control of visual perception: Cortical and subcortical mech- anisms. Cold Spring Harbor Symposium on Quantitative Biology, 55, 963-971.

Deubel, H., \& Schneider, W. X. (1996). Saccade target selection and object recognition: Evidence for a common mechanism. Vision Research, 36, $1827-1837$

De Weerd, P., Peralta, M. R., III, Desimone, R., \& Ungerleider, L. G. (1999). Loss of attentional stimulus selection after extrastriate cortical lesions in macaques. Nature Neuroscience, 2, 753-758.

Di Russo, F., Martinez, A., Sereno, M. I., Pitzalis, S., \& Hillyard, S. A. (2001). Cortical sources of the early components of the visual evoked potential. Human Brain Mapping, 5, 95-111.

Distler, C., Boussaoud, D., Desimone, R., \& Ungerleider, L. G. (1993). Cortical connections of inferior temporal area TEO in macaque monkeys. Journal of Comparative Neurology, 334, 125-150.

Donk, M., \& Theeuwes, J. (2001). Visual marking beside the mark: Prioritizing selection by abrupt onsets. Perception \& Psychophysics, 63, 891-900.

Downing, C. J., \& Pinker, S. (1985). The spatial structure of visual attention. In M. I. Posner \& O. S. M. Marin (Eds.), Attention and Performance XI: Mechanisms of attention and visual search (pp. 171188). Hillsdale, NJ: Erlbaum.

Driver, J., \& Baylis, G. C. (1989). Movement and visual attention: The spotlight metaphor breaks down. Journal of Experimental Psychology: Human Perception and Performance, 15, 448-456.

Dubois, J., Hamker, F. H., \& VanRullen, R. (2009). Attentional selection of noncontiguous locations: The spotlight is only transiently "split." Journal of Vision, 9, 1-11.

Duncan, J. (1984). Selective attention and the organization of visual information. Journal of Experimental Psychology, 113, 501-517.

Duncan, J. (1996). Cooperating brain systems in selective perception and action. In M. I. Posner \& O. S. M. Marin (Eds.), Attention and Performance XI: Mechanisms of attention and visual search (pp. 205-219). Hillsdale, NJ: Erlbaum.

Duncan, J., Ward, R., \& Shapiro, K. L. (1994). Direct measurement of attentional dwell time in human vision. Nature, 369, 313-315.

Egeth, H. E. (1966). Parallel versus serial processes in multidimensional stimulus discrimination. Perception \& Psychophysics, 1, 245-252.

Egeth, H. E. (1977). Attention and pre-attention. In G. E. Bower (Ed.), The psychology of learning and motivation (Vol. 11, pp. 277-320). New York, NY: Academic Press.

Egeth, H. E., \& Yantis, S. (1997). Visual attention: Control, representation, and time course. Annual Review of Psychology, 48, 269-297.

Egly, R., Driver, J., \& Rafal, R. D. (1994). Shifting visual attention between objects and locations: Evidence from normal and parietal lesion subjects. Journal of Experimental Psychology: General, 123, 161-177.

Egly, R., \& Homa, D. (1984). Sensitization of the visual field. Journal of Experimental Psychology: Human Perception and Performance, 10, $778-$ 793.

Eimer, M. (1999). Attending to quadrants and ring-shaped regions: ERP effects of visual attention in different spatial selection tasks. Psychophysiology, 36, 491-503.

Eimer, M. (2000). The time course of spatial orienting elicited by central and peripheral cues: Evidence from event-related brain potentials. Biological Psychology, 53, 253-258.

Engbert, R., Nuthmann, A., Richter, E. M., \& Kliegl, R. (2005). SWIFT: A dynamical model of saccade generation during reading. Psychological Review, 112, 777-813.

Engel, A. K., Fries, P., \& Singer, W. (2001). Dynamic predictions: Oscillations and synchrony in top-down processing. Nature Reviews Neuroscience, 2, 704-716.

Enns, J. T., Austen, E. L., Di Lollo, V., Rauschenberger, R., \& Yantis, S. (2001). New objects dominate luminance transients in setting attentional priority. Journal of Experimental Psychology: Human Perception and Performance, 27, 1287-1302. 
Enns, J. T., \& Di Lollo, V. (2000). What's new in visual masking? Trends in Cognitive Sciences, 4, 345-352.

Eriksen, B. A., \& Eriksen, C. W. (1974). Effects of noise letters upon the identification of a target letter in a nonsearch task. Perception \& Psychophysics, 16, 433-149.

Eriksen, C. W., \& Murphy, T. D. (1987). Movement of attentional focus across the visual field: A critical look at the evidence. Perception \& Psychophysics, 42, 299-305.

Eriksen, C. W., \& St. James, J. D. (1986). Visual attention within and around the field of focal attention: A zoom lens model. Perception \& Psychophysics, 40, 225-240.

Eriksen, C. W., \& Yeh, Y.-Y. (1985). Allocation of attention in the visual field. Journal of Experimental Psychology: Human Perception and Performance, 11, 583-597.

Fehd, H. M., \& Seiffert, A. E. (2007). Eye movements during multiple object tracking: Where do participants look? Cognition, 108, 201-209.

Felleman, D. J., \& Van Essen, C. C. (1991). Distributed hierarchical processing in the primate cerebral cortex. Cerebral Cortex, 1, 1-47.

Flombaum, J. I., \& Scholl, B. J. (2008). How does attention operate during multiple object tracking? Evidence from the "slot-machine" task for parallel access to target features [Abstract]. Journal of Vision, 8(6), 223a.

Flombaum, J. I., Scholl, B. J., \& Pylyshyn, Z. W. (2008). Attentional resources in visual tracking through occlusion: The high-beams effect. Cognition, 107, 904-931.

Folk, C. L., Remington, R. W., \& Johnston, J. C. (1992). Involuntary covert orienting is contingent on attentional control settings. Journal of Experimental Psychology: Human Perception and Performance, 18, $1030-1044$.

Forster, S., \& Lavie, N. (2008). Failures to ignore entirely irrelevant distractors: The role of load. Journal of Experimental Psychology: Applied, 14, 73-83.

Franconeri, S. L., \& Simons, D. J. (2005). The dynamic events that capture visual attention: A reply to Abrams and Christ (2005). Perception \& Psychophysics, 67, 962-966.

Frey, R. D. (1990). Selective attention, event perception and the criterion of acceptability principle: Evidence supporting and rejecting the doctrine of prior entry. Human Movement Science, 9, 481-530.

Fries, P. (2005). A mechanism for cognitive dynamics: Neuronal communication through neuronal coherence. Trends in Cognitive Sciences, 9, 474480.

Fries, P., Nikolić, D., \& Singer, W. (2007). The gamma cycle. Trends in Neurosciences, 30, 309-316.

Fries, P., Reynolds, J. H., Rorie, A. E., \& Desimone, R. (2001). Modulation of oscillatory neuronal synchronization by selective visual attention. Science, 291, 1560-1563.

Fries, P., Womelsdorf, T., Oostenveld, R., \& Desimone, R. (2008). The effects of visual stimulation and selective visual attention on rhythmic neuronal synchronization in macaque area V4. Journal of Neuroscience, 28, 4823-4835.

Fu, S., Caggiano, D. M., Greenwood, P. M., \& Parasuraman, R. (2005). Event-related potentials reveal dissociable mechanisms for orienting and focusing visuospatial attention. Cognitive Brain Research, 23, 341-353.

Gallant, J. L., Shoup, R. E., \& Mazer, J. A. (2000). A human extrastriate area functionally homologous to macaque V4. Neuron, 27, 227-235.

Gattass, R., Gross, C. G., \& Sandell, J. H. (1981). Visual topography of V2 in the macaque. Journal of Comparative Neurology, 201, 519-539.

Gattass, R., Oswaldo-Cruz, E., \& Sousa, A. P. (1979). Visual receptive fields of units in the pulvinar of cebus monkey. Brain Research, 160, 413-430.

Gobell, J. L., Tseng, C., \& Sperling, G. (2004). The spatial distribution of visual attention. Vision Research, 44, 1273-1296.

Godijn, R., \& Theeuwes, J. (2003). Parallel allocation of attention prior to the execution of saccade sequences. Journal of Experimental Psychology: Human Perception and Performance, 29, 882-896.

Goldberg, M. E., \& Wurtz, R. H. (1972). Activity of superior colliculus in behaving monkey: I. Visual receptive fields of single neurons. Journal of Neurophysiology, 35, 542-559.

Goodale, M. A., \& Milner, A. D. (1992). Separate visual pathways for perception and action. Trends in Neurosciences, 15, 20-25.

Gopher, D. (1986). In defense of resources: On structures, energies, pools and the allocation of attention. In R. J. Hockey, A. W. K. Gaillard, \& M. G. H. Coles (Eds.), Energetics and human information processing (pp. 353-371). Dordrecht, the Netherlands: Nijhoff.

Gottlieb, J. P., Kusunoki, M., \& Goldberg, M. E. (1998). The representation of visual salience in monkey parietal cortex. Nature, 391, 481-484.

Gowen, E., Abadi, R. V., \& Poliakoff, E. (2005). Paying attention to saccadic intrusions. Cognitive Brain Research, 25, 810-825.

Gowen, E., Abadi, R. V., Poliakoff, E., Hansen, P. C., \& Miall, R. C. (2007). Modulation of saccadic intrusions by exogenous and endogenous attention. Brain Research, 1141, 154-167.

Gray, C. M. (1999). The temporal correlation hypothesis of visual feature integration: Still alive and well. Neuron, 24, 31-47.

Graziano, M. S. (2001). Is reaching eye-centered, body-centered, handcentered, or a combination? Reviews in the Neurosciences, 12, 175-185.

Gregoriou, G. G., Gotts, S. J., Zhou, H., \& Desimone, R. (2009). Highfrequency, long-range coupling between prefrontal and visual cortex during attention. Science, 324, 1207-1210.

Gross, C. G., Bender, D. B., \& Mishkin, M. (1977). Contributions of the corpus callosum and the anterior commissure to visual activation of inferior temporal neurons. Brain Research, 131, 227-239.

Gross, C. G., Rocha-Miranda, C. E., \& Bender, D. B. (1972). Visual properties of neurons in inferotemporal cortex of the macaque. Journal of Neurophysiology, 35, 96-111.

Grossberg, S. (1973). Contour enhancement, short term memory, and constancies in reverberating neural networks. Studies in Applied Mathematics, 52, 213-257.

Günay, C., \& Maida, A. S. (2003). Temporal binding as an inducer for connectionist recruitment learning over delayed lines. Neural Networks, $16,593-600$

Hahn, S., \& Kramer, A. (1998). Further evidence for the division of attention among non-contiguous locations. Visual Cognition, 5, 217-256.

Hasenstaub, A., Shu, Y., Haider, B., Kraushaar, U., Duque, A., \& McCormick, D. A. (2005). Inhibitory postsynaptic potentials carry synchronized frequency information in active cortical networks. Neuron, 47, 423-435.

Heinze, H.-J., Luck, S. J., Münte, T. F., Gös, A., Mangun, G. R., \& Hillyard, S. A. (1994). Attention to adjacent and separate positions in space: An electrophysiological analysis. Perception \& Psychophysics, $56,42-52$.

Henderson, J. M. (1992). Visual attention and eye movement control during reading and picture viewing. In K. Rayner (Ed.), Eye movements and visual cognition: Scene perception and reading (pp. 261-283). New York, NY: Springer-Verlag.

Henderson, J. M., \& Macquistan, A. D. (1993). The spatial distribution of attention following an exogenous cue. Perception \& Psychophysics, 53, 221-230.

Henderson, J. M., Pollatsek, A., \& Rayner, K. (1989). Covert visual attention and extrafoveal information use object identification. Perception \& Psychophysics, 45, 196-208.

Hillyard, S. A., \& Anllo-Vento, L. (1998). Event-related brain potentials in the study of visual selective attention. Proceedings of the National Academy of Sciences, USA, 95, 781-787.

Holtzman, J. D. (1981). Interactions between cortical and subcortical visual areas: Evidence from human commissurotomy patients. Visual Research, 24, 801-813.

Hopf, J.-M., Boelmans, K., Schoenfeld, M. A., Luck, S. J., \& Heinze, H.-J. (2004). Attention to features precedes attention to locations in visual search: Evidence from electromagnetic brain responses in humans. Journal of Neuroscience, 24, 1822-1832.

Hopf, J.-M., Luck, S. J., Girelli, M., Hagner, T., Mangun, G. R., Scheich, 
H., \& Heinze, H.-J. (2000). Neural sources of focused attention in visual search. Cerebral Cortex, 10, 1233-1241.

Houtkamp, R., \& Roelfsema, P. R. (2009). Matching of visual input to only one item at any one time. Psychological Research, 73, 317-326.

Hughes, H. C., \& Zimba, L. D. (1985). Spatial maps of directed visual attention. Journal of Experimental Psychology: Human Perception and Performance, 11, 409-430.

Hughes, H. C., \& Zimba, L. D. (1987). Natural boundaries for the spatial spread of directed visual attention. Neuropsychologia, 25, 5-18.

Intriligator, J., \& Cavanagh, P. (2001). The spatial resolution of visual attention. Cognitive Psychology, 43, 171-216.

Jaskowski, P., van der Lubbe, R. H. J., Schlotterbeck, E., \& Verleger, R. (2002). Traces left on visual selective attention by stimuli that are not consciously identified. Psychological Science, 13, 48-54.

Johansson, R. S., Westling, G., Bäckström, A., \& Flanagan, J. R. (2001). Eye-hand coordination in object manipulation. Journal of Neuroscience, 21, 6917-6932.

Jonides, J., \& Yantis, S. (1988). Uniqueness of abrupt visual onset in capturing attention. Perception \& Psychophysics, 43, 346-354.

Jordan, T. R., \& Patching, G. R. (2006). Assessing effects of fixation demands on perception of lateralized words: A visual window technique for studying hemispheric asymmetry. Neuropsychologia, 44, 686-692.

Jordan, T. R., Patching, G. R., \& Milner, A. D. (1998). Central fixations are inadequately controlled by instructions alone: Implications for studying cerebral asymmetry. Quarterly Journal of Experimental Psychology: Human Experimental Psychology, 51A, 371-391.

Jordan, T. R., Patching, G. R., \& Milner, A. D. (2000). Lateralized word recognition: Assessing the role of hemispheric specialization, modes of lexical access, and perceptual asymmetry. Journal of Experimental Psychology: Human Perception and Performance, 26, 1192-1208.

Jordan, T. R., Patching, G. R., \& Thomas, S. M. (2003). Assessing the role of hemispheric specialization, serial-position processing and retinal eccentricity in lateralized word perception. Cognitive Neuropsychology, $20,49-71$.

Joseph, J. S., Chun, M. M., \& Nakayama, K. (1997). Attentional requirements in a "preattentive" feature search task. Nature, 387, 805-807.

Juan, C.-H., Shorter-Jacobi, S. M., \& Schall, J. D. (2004). Dissociation of spatial attention and saccade preparation. Proceedings of the National Academy of Sciences, USA, 101, 15541-15544.

Juola, J. F., Bouwhuis, D. G., Cooper, E. E., \& Warner, C. B. (1991). Control of attention around the fovea. Journal of Experimental Psychology: Human Perception and Performance, 17, 125-141.

Juola, J. F., Koshino, H., \& Warner, C. B. (1995). Tradeoffs between attentional effects of spatial cues and abrupt onsets. Perception \& Psychophysics, 57, 333-342.

Kahneman, D. (1973). Attention and effort. Englewood Cliffs, NJ: Prentice Hall.

Kahneman, D., \& Treisman, A. (1984). Changing views of attention and automaticity. In R. Parasuraman \& D. R. Davies (Eds.), Varieties of attention (pp. 29-61). New York, NY: Academic Press.

Kahneman, D., Treisman, A., \& Gibbs, B. J. (1992). The reviewing of object files: Object-specific integration of information. Cognitive Psychology, 24, 174-219.

Karni, A., \& Bertini, G. (1997). Learning perceptual skills: Behavioral probes into adult cortical plasticity. Current Opinion in Neurobiology, 7 , $530-535$.

Karni, A., \& Sagi, D. (1991). Where practice makes perfect in texture discrimination: Evidence for primary visual cortex plasticity. Proceedings of the National Academy of Sciences, USA, 88, 4966-4970.

Kastner, S., De Weerd, P., Desimone, R., \& Ungerleider, L. G. (1998). Mechanisms of directed attention in the human extrastriate cortex as revealed by functional MRI. Science, 282, 108-111.

Kastner, S., Pinsk, M. A., De Weerd, P., Desimone, R., \& Ungerleider,
L. G. (1999). Increased activity in human visual cortex during directed attention in the absence of visual stimulation. Neuron, 22, 751-761.

Kawahara, J.-I., \& Yamada, Y. (2006). Two noncontiguous locations can be attended concurrently: Evidence from the attentional blink. Psychonomic Bulletin \& Review, 13, 594-599.

Khayat, P. S., Spekreijse, H., \& Roelfsema, P. R. (2006). Attention lights up new object representations before the old ones fade away. Journal of Neuroscience, 26, 138-142.

Kim, S.-G., Richter, W., \& Uğurbil, K. (1997). Limitations of temporal resolution in functional MRI. Magnetic Resonance in Medicine, 37, 631-636.

Klein, R. M. (1980). Does oculomotor readiness mediate cognitive control of visual attention? In R. S. Nickerson (Ed.), Attention and performance VIII (pp. 259-276). Hillsdale, NJ: Erlbaum.

Klotz, W., \& Neumann, O. (1999). Motor activation without conscious discrimination in metacontrast masking. Journal of Experimental Psychology: Human Perception and Performance, 25, 976-992.

Koch, C., \& Ullman, S. (1985). Shifts in selective visual attention: Towards the underlying neural circuitry. Human Neurobiology, 4, 219-227.

Kowler, E., Anderson, E., Dosher, B., \& Blaser, E. (1995). The role of attention in the programming of saccades. Vision Research, 35, 1897-1916.

Kraft, A., Müller, N. G., Hagendorf, H., Schira, M. M., Dick, S., Fendrich, R. M., \& Brandt, S. A. (2004). Interactions between task difficulty and hemispheric distribution of attended locations: Implications for the splitting attention debate. Cognitive Brain Research, 24, 19-32.

Kramer, A. F., \& Hahn, S. (1995). Splitting the beam: Distribution of attention over noncontiguous regions of the visual field. Psychological Science, 6, 381-386.

Kröse, B. J., \& Julesz, B. (1989). The control and speed of shifts of attention. Vision Research, 29, 1607-1619.

Kusunoki, M., Gottlieb, J., \& Goldberg, M. E. (2000). The lateral intraparietal area as a salience map: The representation of abrupt onset, stimulus motion, and task relevance. Vision Research, 40, 1459-1468.

Kutas, M., \& Dale, A. (1997). Electrical and magnetic readings of mental functions. In M. D. Rugg (Ed.), Cognitive neuroscience: Studies in cognition (pp. 197-237). King's Lynn, England: Biddles.

LaBerge, D. (1983). Spatial extent of attention to letters and words. Journal of Experimental Psychology: Human Perception and Performance, 9, 371-379.

LaBerge, D., \& Brown, V. (1986). Variations in size of the visual field in which targets are presented: An attentional range effect. Perception \& Psychophysics, 40, 188-200.

LaBerge, D., \& Brown, V. (1989). Theory of attentional operations in shape identification. Psychological Review, 96, 101-124.

Lachter, J., Forster, K. I., \& Ruthruff, E. (2004). Forty-five years after Broadbent (1958): Still no identification without attention. Psychological Review, 111, 880-913.

Larson, E. B., \& Brown, W. S. (1997). Bilateral field interactions, hemispheric specialization and evoked potential interhemispheric transmission time. Neuropsychologia, 35, 573-581.

Lavie, N. (1995). Perceptual load as a necessary condition for selective attention. Journal of Experimental Psychology: Human Perception and Performance, 21, 451-468.

Lavie, N., \& Cox, S. (1997). On the efficiency of visual selective attention: Efficient visual search leads to inefficient distractor rejection. Psychological Science, 8, 395-398.

Lavie, N., \& Tsal, Y. (1994). Perceptual load as a major determinant of the locus of selection in visual attention. Perception \& Psychophysics, 56 183-197.

Lomber, S. G., Payne, B. R., Hilgetag, C. C., \& Rushmore, R. J. (2002). Restoration of visual orienting into a cortically blind hemifield by reversible deactivation of posterior parietal cortex or the superior colliculus. Experimental Brain Research, 142, 463-474.

Luck, S. J. (2004). Ten simple rules for designing ERP experiments. In 
T. C. Handy (Ed.), Event-related potentials: A methods handbook (pp. 17-32). Cambridge, MA: MIT Press.

Luck, S. J., Chelazzi, L., Hillyard, S. A., \& Desimone, R. (1997). Neural mechanisms of spatial selective attention in areas V1, V2, and V4 of macaque visual cortex. Journal of Neurophysiology, 77, 24-42.

Luck, S. J., \& Hillyard, S. A. (1994a). Electrophysiological correlates of feature analysis during visual search. Psychophysiology, 31, 291-308.

Luck, S. J., \& Hillyard, S. A. (1994b). Spatial filtering during visual search: Evidence from human electrophysiology. Journal of Experimental Psychology: Human Perception and Performance, 20, 1000-1014.

Luck, S. J., Hillyard, S. A., Mangun, G. R., \& Gazzaniga, M. S. (1989). Independent hemispheric attentional systems mediate visual search in split-brain patients. Nature, 342, 543-545.

Lueschow, A., Miller, E. K., \& Desimone, R. (1994). Inferior temporal mechanisms for invariant object recognition. Cerebral Cortex, 4, 523531

Lyon, D. C., Jain, N., \& Kaas, J. H. (2003). The visual pulvinar in tree shrews: II. Projections of four nuclei to areas of visual cortex. Journal of Comparative Neurology, 467, 607-627.

Macaluso, E., \& Patria, F. (2007). Spatial re-orienting of visual attention along the horizontal or the vertical axis. Experimental Brain Research, 180, 23-34

Makovski, T., Vázquez, G. A., \& Jiang, Y. V. (2008). Visual learning in multiple-object tracking. PLoS ONE, 3, e2228. doi:10.1371/journal .pone.0002228

Malinowski, P., Fuchs, S., \& Müller, M. M. (2007). Sustained division of spatial attention to multiple locations within one hemifield. Neuroscience Letters, 414, 65-70.

Mangun, G. R. (1995). Neural mechanisms of visual selective attention. Psychophysiology, 32, 4-18.

Mangun, G. R., Luck, S. J., Plager, R., Loftus, W., Hilllyard, S. A., \& Handy, T. (1994). Monitoring the visual world: Hemispheric asymmetries and subcortical processes in attention. Journal of Cognitive Neuroscience, 6, 267-275.

Martin, E. W., \& Shapiro, K. L. (2008). Does failure to mask T1 cause lag-1 sparing in the attentional blink? Perception \& Psychophysics, 70, 562-570.

Matsubara, K., Shioiri, S., \& Yaguchi, H. (2007). Spatial spread of visual attention while tracking a moving object. Optical Review, 14, 57-63.

Mattingley, J. B., Davis, G., \& Driver, J. (1997). Preattentive filling-in of visual surfaces in parietal extinction. Science, 275, 671-673.

Maunsell, J. H. R., \& Treue, S. (2006). Feature-based attention in visual cortex. Trends in Neurosciences, 29, 317-322.

Mazer, J. A., \& Gallant, J. L. (2003). Goal-related activity in V4 during free viewing visual search evidence for a ventral stream visual salience map. Neuron, 40, 1241-1250.

McCormick, P. A. (1997). Orienting attention without awareness. Journal of Experimental Psychology: Human Perception and Performance, 23, $168-180$

McCormick, P. A., \& Klein, R. (1990). The spatial distribution of attention during covert visual orienting. Acta Psychologica, 75, 225-242.

McCormick, P. A., Klein, R. M., \& Johnston, S. (1998). Splitting versus sharing focal attention: Comment on Castiello and Umiltà (1992). Journal of Experimental Psychology: Human Perception and Performance, $24,350-357$

McIlwain, J. (1973). Retinotopic fidelity of striate cortex-superior colliculus interactions in the cat. Journal of Neurophysiology, 36, 702-710.

McMains, S. A., \& Somers, D. C. (2004). Multiple spotlights of attentional selection in human visual cortex. Neuron, 42, 677-686.

McMains, S. A., \& Somers, D. C. (2005). Processing efficiency of divided spatial attention mechanisms in human visual cortex. Journal of Neuroscience, 25, 9444-9448.

Menon, R. S., \& Kim, S.-G. (1999). Spatial and temporal limits in cognitive neuroimaging with fMRI. Trends in Cognitive Sciences, 3, 207-216.
Miller, J. (1988). Discrete and continuous models of human information processing: Theoretical distinctions and empirical results. Acta Psychologica, 67, 191-257.

Miller, J. (1991). The flanker compatibility effect as a function of visual angle, attentional focus, visual transients, and perceptual load: A search for boundary conditions. Perception \& Psychophysics, 49, 270-288.

Mordkoff, J. T., Yantis, S., \& Egeth, H. E. (1990). Detecting conjunctions of color and form in parallel. Perception \& Psychophysics, 48, 157-168.

Moore, C. M., Egeth, H., Berglan, L. R., \& Luck, S. J. (1996). Are attentional dwell times inconsistent with serial visual search? Psychonomic Bulletin \& Review, 3, 360-365.

Moore, T., \& Armstrong, K. M. (2003). Selective gating of visual signals by microstimulation of frontal cortex. Nature, 421, 370-373.

Moore, T., \& Fallah, M. (2004). Microstimulation of the frontal eye field and its effects on covert spatial attention. Journal of Neurophysiology, 91, 152-162.

Moran, J., \& Desimone, R. (1985). Selective attention gates visual processing in the extrastriate cortex. Science, 229, 782-784.

Morgan, S. T., Hansen, J. C., \& Hillyard, S. A. (1996). Selective attention to stimulus location modulates the steady-state visual evoked potential. Proceedings of the National Academy of Sciences, USA, 93, 4770-4774.

Morrison, R. E. (1984). Manipulation of stimulus onset delay in reading: Evidence for parallel programming of saccades. Journal of Experimental Psychology: Human Perception and Performance, 10, 667-682.

Morrone, M. C., Denti, V., \& Spinelli, D. (2002). Color and luminance contrasts attract independent attention. Current Biology, 12, 1134-1137.

Morrone, M. C., Denti, V., \& Spinelli, D. (2004). Different attentional resources modulate the gain mechanisms for color and luminance contrast. Vision Research, 44, 1389-1401.

Motter, B. C. (1993). Focal attention produces spatially selective processing in visual cortical areas V1, V2, and V4 in the presence of competing stimuli. Journal of Neurophysiology, 70, 909-919.

Motter, B. C. (1994a). Neural correlates of attentive selection for color or luminance in extrastriate area V4. Journal of Neuroscience, 14, 2178 2189.

Motter, B. C. (1994b). Neural correlates of feature selective memory and pop-out in extrastriate area V4. Journal of Neuroscience, 14, 2190 2199.

Müller, H. J., \& Ebeling, D. (2008). Attention-modulated activity in visual cortex-More than a simple "spotlight." NeuroImage, 40, 818-827.

Müller, H. J., \& Findlay, J. M. (1987). Sensitivity and criterion effects in the spatial cuing of visual attention. Perception \& Psychophysics, 42, 383-399.

Müller, M. M., \& Hübner, R. (2002). Can the spotlight of attention be shaped like a doughnut? Evidence from steady-state visual evoked potentials. Psychological Science, 13, 119-124.

Müller, M. M., Malinowski, P., Gruber, T., \& Hillyard, S. A. (2003). Sustained division of the attentional spotlight. Nature, 424, 309-312.

Müller, M. M., Picton, T. W., Valdes-Sosa, P., Riera, J., Teder-Sälejärvi, W. A., \& Hillyard, S. A. (1998). Effects of spatial selective attention on the steady-state visual evoked potential in the $20-28 \mathrm{~Hz}$ range. Cognitive Brain Research, 6, 249-261.

Müller, M. M., Teder-Sälejärvi, W., \& Hillyard, S. A. (1998). The time course of cortical facilitation during cued shifts of spatial attention. Nature Neuroscience, 1, 631-634.

Müller, N. G., Bartelt, O. A., Donner, T. H., Villringer, A., \& Brandt, S. A (2003). A physiological correlate of the "zoom lens" of visual attention. Journal of Neuroscience, 23, 3561-3565.

Müller, N. G., \& Kleinschmidt, A. (2004). The attentional "spotlight's" penumbra: Center-surround modulation in striate cortex. NeuroReport, 15, 977-980.

Müller, N. G., Mollenhauer, M., Rösler, A., \& Kleinschmidt, A. (2005). The attentional field has a Mexican hat distribution. Vision Research, 45, $1129-1137$. 
Navon, D., \& Gopher, D. (1979). On the economy of the human-processing system. Psychological Review, 86, 214-255.

Neitzel, S., Mandon, S., \& Kreiter, A. K. (2009, September-October). Attention modulates the phase coherence between macaque visual areas V1 and V4. Poster presented at the Bernstein Conference on Computational Neuroscience, Frankfurt am Main, Germany. doi:10.3389/ conf.neuro.10.2009.14.061

Nobre, A. C., Rao, A., \& Chelazzi, L. (2006). Selective attention to specific features within objects: Behavioral and electrophysiological evidence. Journal of Cognitive Neuroscience, 18, 539-561.

Nomura, M., Katahata, M., \& Hashiya, K. (2005). Visual orienting occurs asymmetrically in horizontal vs. vertical planes. Psychologia, 48, 205217.

O'Craven, K. M., Downing, P. E., \& Kanwisher, N. (1999). fMRI evidence for objects as units of attentional selection. Nature, 401, 584-587.

Oksama, L., \& Hyönä, J. (2004). Is multiple object tracking carried out automatically by an early vision mechanism independent of higher-order cognition? An individual difference approach. Visual Cognition, 11, 631-671.

Olson, C. R. (2003). Brain representation of object-centered space in monkeys and humans. Annual Review of Neuroscience, 26, 331-354.

Pan, K., \& Eriksen, C. W. (1993). Attentional distribution in the visual field during same-different judgments as assessed by response competition. Perception \& Psychophysics, 53, 134-144.

Pashler, H., \& Johnston, J. C. (1998). Attentional limitations in dual-task performance. In H. Pashler (Ed.), Attention (pp. 155-189). Philadelphia, PA: Taylor \& Francis.

Pavlovskaya, M., Ring, H., Groswasser, Z., Keren, O., \& Hochstei, S. (2001). Visual search in peripheral vision: Learning effects and set-size dependence. Spatial Vision, 14, 151-173.

Payne, B. R., Lomber, S. G., Geeraerts, S., van der Gucht, E., \& Vandenbussche, E. (1996). Reversible visual hemineglect. Proceedings of the National Academy of Sciences, USA, 93, 290-294.

Petersen, S. E., Robinson, D. L., \& Morris, J. D. (1987). Contributions of the pulvinar to visual spatial attention. Neuropsychologia, 25, 97-105.

Peterson, M. S., \& Juola, J. F. (2000). Evidence for distinct attentional bottlenecks in attention switching and attentional blink tasks. Journal of General Psychology, 127, 6-26.

Pollatsek, A., Reichle, E. D., \& Rayner, K. (2006). Attention to one word at a time in reading is still a viable hypothesis: Rejoinder to Inhoff, Radach, and Eiter (2006). Journal of Experimental Psychology: Human Perception and Performance, 32, 1496-1500.

Popper, K. (1959). The logic of scientific discovery. New York, NY: Basic Books.

Posner, M. I. (1980). Orienting of attention. Quarterly Journal of Experimental Psychology, 32, 3-25.

Posner, M. I., \& Cohen, Y. (1984). Components of visual orienting. In H. Bouma \& D. G. Bouwhuis (Eds.), Attention and performance $X$ (pp. 531-556). Hillsdale, NJ: Erlbaum.

Posner, M. I., Snyder, C. R., \& Davidson, B. J. (1980). Attention and the detection of signals. Journal of Experimental Psychology: General, 109, $160-174$

Potter, M. C., Chun, M. M., Banks, B. S., \& Muckenhoupt, M. (1998). Two attentional deficits in serial target search: The visual attentional blink and an amodal task-switch deficit. Journal of Experimental Psychology: Learning, Memory, and Cognition, 24, 979-992.

Pouget, A., \& Sejnowski, T. J. (2001). Simulating a lesion in a basis function model of spatial representations: Comparison with hemineglect. Psychological Review, 108, 653-673.

Pratt, J., \& Quilty, L. (2002). Examining the activity-distribution model of visual attention with exogenous cues and targets. Quarterly Journal of Experimental Psychology: Human Experimental Psychology, 55A, 627641
Pylyshyn, Z. (1989). The role of location indexes in spatial perception: A sketch of the FINST spatial-index model. Cognition, 32, 65-97.

Pylyshyn, Z. W. (2000). Situating vision in the world. Trends in Cognitive Sciences, 4, 197-207.

Pylyshyn, Z. W. (2001). Visual indexes, preconceptual objects, and situated vision. Cognition, 80, 127-158.

Pylyshyn, Z. W. (2003). Seeing and visualizing: It's not what you think. Cambridge, MA: MIT Press.

Pylyshyn, Z. W. (2006). Some puzzling findings in multiple object tracking (MOT): II. Inhibition of moving nontargets. Visual Cognition, 14(2), 175-198.

Pylyshyn, Z. W. (2007). Things and places: How the mind connects with the world. Cambridge, MA: MIT Press.

Pylyshyn, Z. W., \& Annan, V., Jr. (2006). Dynamics of target selection in Multiple Object Tracking (MOT). Spatial Vision, 19, 485-504.

Pylyshyn, Z. W., \& Storm, R. W. (1988). Tracking multiple independent targets: Evidence for a parallel tracking mechanism. Spatial Vision, 3, 179-197.

Quiroga, R. Q., Snyder, L. H., Batista, A. P., Cui, H., \& Andersen, R. A. (2006). Movement intention is better predicted than attention in the posterior parietal cortex. Journal of Neuroscience, 26, 3615-3620.

Rao, S. C., Rainer, G., \& Miller, E. K. (1997). Integration of what and where in the primate prefrontal cortex. Science, 276, 821-824.

Raymond, J. E., Shapiro, K. L., \& Arnell, K. M. (1992). Temporary suppression of visual processing in an RSVP task: An attentional blink? Journal of Experimental Psychology: Human Perception and Performance, 18, 849-860.

Reeves, A., \& Sperling, G. (1986). Attention gating in short-term visual memory. Psychological Review, 93, 180-206.

Reichle, E. D., Pollatsek, A., Fisher, D. L., \& Rayner, K. (1998). Toward a model of eye movement control in reading. Psychological Review, 105, $125-157$.

Reinagel, P., \& Zador, A. M. (1999). Natural scene statistics at the centre of gaze. Network: Computation in Neural Systems, 10, 341-350.

Reynolds, J. H., Chelazzi, L., \& Desimone, R. (1999). Competitive mechanisms subserve attention in macaque areas V2 and V4. Journal of Neuroscience, 19, 1736-1753.

Reynolds, J. H., \& Heeger, D. J. (2009). The normalization model of attention. Neuron, 61, 168-185.

Rizzolatti, G. (1983). Mechanisms of selective attention in mammals. In J.-P. Ewert, R. R. Capranica, \& D. J. Ingle (Eds.), Advances in vertebrate neuroethology (pp. 261-297). London, England: Plenum Press.

Rizzolatti, G., Riggio, L., Dascola, I., \& Umiltà, C. (1987). Reorienting attention across the horizontal and vertical meridians: Evidence in favor of a premotor theory of attention. Neuropsychologia, 25, 31-40.

Roelfsema, P. R., Khayat, P. S., \& Spekreijse, H. (2003). Subtask sequencing in the primary visual cortex. Proceedings of the National Academy of Sciences, USA, 100, 5467-5472.

Roelfsema, P. R., Lamme, V. A., \& Spekreijse, H. (1998). Object-based attention in the primary visual cortex of the macaque monkey. Nature 395, 376-381.

Rollenhagen, J. E., \& Olson, C. R. (2005). Low-frequency oscillations arising from competitive interactions between visual stimuli in macaque inferotemporal cortex. Journal of Neurophysiology, 94, 3368-3387.

Ruthruff, E., Pashler, H. E., \& Hazeltine, E. (2003). Dual-task interference with equal task emphasis: Graded capacity sharing or central postponement? Perception \& Psychophysics, 65, 801-816.

Saalmann, Y. B., Pigarev, I. N., \& Vidyasagar, T. R. (2007). Neural mechanisms of visual attention: How top-down feedback highlights relevant locations. Science, 316, 1612-1615.

Saarinen, J., \& Levi, D. M. (2000). Perception of mirror symmetry reveals long-range interactions between orientation-selective cortical filters NeuroReport, 11, 2133-2138.

Sack, A. T., Sperling, J. M., Prvulovic, D., Formisano, E., Goebel, R., Di 
Salle, F., ... Linden, D. E. (2002). Tracking the mind's image in the brain II: Transcranial magnetic stimulation reveals parietal asymmetry in visuospatial imagery. Neuron, 35, 195-204.

Sàenz, M., Buracas, G. T., \& Boynton, G. M. (2003). Global feature-based attention for motion and color. Vision Research, 43, 629-637.

Sagi, D., \& Julesz, B. (1985). "Where" and "what" in vision. Science, 228, $1217-1219$

Sagi, D., \& Julesz, B. (1986). Enhanced detection in the aperture of focal attention during simple discrimination tasks. Nature, 321, 693-695.

Scharlau, I. (2004). Evidence for split foci of attention in a priming paradigm. Perception \& Psychophysics, 66, 988-1002.

Scharlau, I., \& Ansorge, U. (2003). Direct parameter specification of an attention shift: Evidence from perceptual latency priming. Vision Research, 43, 1351-1363.

Scharlau, I., \& Neumann, O. (2003a). Perceptual latency priming by masked and unmasked stimuli: Evidence for an attentional interpretation. Psychological Research, 67, 184-196.

Scharlau, I., \& Neumann, O. (2003b). Temporal Parameters and the time course of perceptual latency priming. Acta Psychologica, 113, 185-203.

Schenkluhn, B. M., Ruff, C. C., Heinen, K., \& Chambers, C. D. (2008). Parietal stimulation decouples spatial and feature-based attention. Journal of Neuroscience, 28, 11106-11110.

Schneider, K. A., \& Bavelier, D. (2003). Components of visual prior entry. Cognitive Psychology, 47, 333-366.

Scholl, B. J., \& Pylyshyn, Z. W. (1999). Tracking multiple items through occlusion: Clues to visual objecthood. Cognitive Psychology, 38, 259290

Scialfa, C. T., Jenkins, L., Hamaluk, E., \& Skaloud, P. (2000). Aging and the development of automaticity in conjunction search. Journals of Gerontology: Series B. Psychological Sciences and Social Sciences, 55, P27-P46.

Sereno, A. B. (1992). Programming saccades: The role of attention. In K. Rayner (Ed.), Eye movements and visual cognition: Scene perception and reading (pp. 304-316). New York, NY: Springer-Verlag.

Shapiro, K. L., Arnell, K., \& Raymond, J. E. (1997). The attentional blink: A view on attention and a glimpse on consciousness. Trends in Cognitive Sciences, 1, 291-295.

Shaw, M. L., \& Shaw, P. (1977). Optimal allocation of cognitive resources to spatial locations. Journal Experimental Psychology: Human Perception and Performance, 3, 201-211.

Sheliga, B. M., Riggio, L., \& Rizzolatti, G. (1994). Orienting of attention and eye movements. Experimental Brain Research, 98, 507-522.

Shiffrin, R. M., \& Gardner, G. T. (1972). Visual processing capacity and attentional control. Journal of Experimental Psychology, 93, 72-82.

Shiffrin, R. M., \& Schneider, W. (1977). Controlled and automatic human information processing: II. Perceptual learning, automatic attending, and a general theory. Psychological Review, 82, 127-190.

Shim, W. M., Alvarez, G. A., \& Jiang, Y. V. (2008). Spatial separation between targets constrains maintenance of attention on multiple objects. Psychonomic Bulletin \& Review, 15, 390-397.

Shmuel, A., Yacoub, E., Pfeuffer, J., Van de Moortele, P. F., Adriany, G., Hu, X., \& Uğurbil, K. (2002). Sustained negative BOLD, blood flow and oxygen consumption response and its coupling to the positive response in the human brain. Neuron, 36, 1195-1210.

Shore, D. I., Spence, C., \& Klein, R. M. (2001). Visual prior entry. Psychological Science, 12, 205-212.

Shulman, G. L., Remington, R. W., \& McLean, J. P. (1979). Moving attention through visual space. Journal of Experimental Psychology: Human Perception and Performance, 5, 522-526.

Singer, W. (2004). Synchrony, oscillations, and relational codes. In L. M. Chalupa \& J. S. Werner (Eds.), The visual neurosciences (Vol. 2, pp. 1665-1681). Cambridge, MA: MIT Press.

Slotnick, S. D., Hopfinger, J. B., Klein, S. A., \& Sutter, E. E. (2002).
Darkness beyond the light: Attentional inhibition surrounding the classic spotlight. NeuroReport, 13, 773-778.

Slotnick, S. D., Schwarzbach, J., \& Yantis, S. (2003). Attentional inhibition of visual processing in human striate and extrastriate cortex. $\mathrm{Neu}-$ roImage, 19, 1602-1611.

Smith, A. T., Singh, K. D., \& Greenlee, M. W. (2000). Attentional suppression of activity in the human visual cortex. NeuroReport, 11, 271-277.

Smith, A. T., Williams, A. L., \& Singh, K. D. (2004). Negative BOLD in the visual cortex: Evidence against blood stealing. Human Brain Mapping, 21, 213-220.

Snyder, J. J., \& Chatterjee, A. (2006). The frontal cortex and exogenous attentional orienting. Journal of Cognitive Neuroscience, 18, 1913-1923.

Snyder, L. A., Batista, A. P., \& Andersen, R. A. (1997). Coding of intention in the posterior parietal cortex. Nature, 386, 167-170.

Somers, D. C., Dale, A. M., Seiffert, A. E., \& Tootell, R. B. (1999). Functional MRI reveals spatially specific attentional modulation in human primary visual cortex. Proceedings of the National Academy of Sciences, USA, 96, 1663-1668.

Sperling, G., \& Reeves, A. (1980). Measuring the reaction time of an unobservable response: A shift of visual attention. In R. S. Nickerson (Ed.), Attention and performance VIII (pp. 347-360). Hillsdale, NJ: Erlbaum.

Sprague, J. M. (1996). Neural mechanisms of visual orienting responses. Progress in Brain Research, 112, 1-15.

Standage, D. I., Trappenberg, T. P., \& Klein, R. M. (2005a, July-August). A continuous attractor neural network model of divided visual attention. Paper presented at the International Joint Conference on Neural Networks, Montreal, Canada.

Standage, D. I., Trappenberg, T. P., \& Klein, R. M. (2005b). Modelling divided visual attention with a winner-take-all network. Neural Networks, 18, 620-627.

Stanton, G. B., Bruce, C. J., \& Goldberg, M. E. (2004). Topography of projections to posterior cortical areas from the macaque frontal eye fields. Journal of Comparative Neurology, 353, 291-305.

Steinman, S. B., \& Steinman, B. A. (1998). Vision and attention. I: Current models of visual attention. Optometry and Vision Science, 75, 146-155.

Sternberg, S. (1966). High-speed scanning in human memory. Science, $153,652-654$.

Theeuwes, J. (1995). Abrupt luminance change pops out; abrupt color change does not. Perception \& Psychophysics, 57, 637-644.

Theeuwes, J., Godijn, R., \& Pratt, J. (2004). A new estimation of the duration of attentional dwell time. Psychonomic Bulletin \& Review, 11, $60-64$.

Thompson, K. G., Biscoe, K. L., \& Sato, T. R. (2005). Neuronal basis of covert spatial attention in the frontal eye field. Journal of Neuroscience, 25, 9479-9487.

Thornton, T. L., \& Gilden, D. L. (2007). Parallel and serial processes in visual search. Psychological Review, 114, 71-103.

Titchener, E. B. (1908). Lectures on the elementary psychology of feeling and attention. New York, NY: Macmillan.

Tootell, R. B., Hadjikhani, N., Hall, E. K., Marrett, S., Vanduffel, W., Vaughan, J. T., \& Dale, A. M. (1998). The retinotopy of visual spatial attention. Neuron, 21, 1409-1422.

Townsend, J. T. (1969). Mock parallel and serial models and experimental detection of these. In Proceedings of the Purdue Centennial Symposium on Information Processing (pp. 617-628). West Lafayette, IN: Purdue University Press.

Townsend, J. T. (1990). Serial vs. parallel processing: Sometimes they look like Tweedledum and Tweedledee but they can (and should) be distinguished. Psychological Science, 1, 46-54.

Townsend, J. T., \& Wenger, M. J. (2004). The serial-parallel dilemma: A case study in a linkage of theory and method. Psychonomic Bulletin \& Review, 11, 391-418.

Treisman, A. (1982). Perceptual grouping and attention in visual search for 
features and objects. Journal of Experimental Psychology: Human Perception and Performance, 8, 194-212.

Treisman, A. M., \& Gelade, G. (1980). A feature-integration theory of attention. Cognitive Psychology, 12, 97-136.

Treisman, A., \& Gormican, S. (1988). Feature analysis in early vision: Evidence from search asymmetries. Psychological Review, 95, 15-48.

Treisman, A., \& Sato, S. (1990). Conjunction search revisited. Journal of Experimental Perception and Performance: Human Perception and Performance, 16, 459-478.

Treue, S., \& Mart'inez Trujillo, J. C. (1999). Feature-based attention influences motion processing gain in macaque visual cortex. Nature, 399, 575-579.

Treue, S., \& Maunsell, J. H. (1999). Effects of attention on the processing of motion in macaque middle temporal and medial superior temporal visual cortex areas. Journal of Neuroscience, 19, 7591-7602.

Tsal, Y. (1983). Movements of attention across the visual field. Journal of Experimental Psychology: Human Perception and Performance, 9, 523530 .

Turatto, M., Benso, F., Facoetti, A., Galfano, G., Mascetti, G. G., \& Umiltà, C. (2000). Automatic and voluntary focusing of attention. Perception \& Psychophysics, 62, 935-952.

Ullman, S. (1984). Visual routines. Cognition, 18, 97-159.

Umiltà, C., Mucignat, C., Riggio, L., Barbieri, C., \& Rizzolatti, G. (1994). Programming shifts of spatial attention. European Journal of Cognitive Psychology, 6, 23-41.

Umiltà, C., Riggio, L., Dascola, I., \& Rizzolatti, G. (1991). Differential effects of central and peripheral cues on the reorienting of spatial attention. European Journal of Cognitive Psychology, 3, 247-267.

Ungerleider, L. G., Galkin, T. W., Desimone, R., \& Gattass, R. (2007). Cortical connections of area V4 in the macaque. Cerebral Cortex, 18, 477-499.

VanRullen, R., Carlson, T., \& Cavanagh, P. (2007). The blinking spotlight of attention. Proceedings of the National Academy of Sciences, USA, 104, 19204-19209.

VanRullen, R., Reddy, L., \& Koch, C. (2004). Visual search and dual tasks reveal two distinct attentional resources. Journal of Cognitive Neuroscience, 16, 4-14.

von der Malsburg, C. (1994). The correlation theory of brain function. In E. Domany, J. L. van Hemmen, \& K. Schulten (Eds.), Models of neural networks II (pp. 95-119). Berlin, Germany: Springer.

Walsh, V., Ellison, A., Ashbridge, E., \& Cowey, A. (1999). The role of the parietal cortex in visual attention-Hemispheric asymmetries and the effects of learning: A magnetic stimulation study. Neuropsychologia, 37, 245-251.
Ward, R., Duncan, J., \& Shapiro, K. (1996). The slow time-course of visual attention. Cognitive Psychology, 30, 79-109.

Weichselgartner, E., \& Sperling, G. (1987). Dynamics of automatic and controlled visual attention. Science, 238, 778-780.

Weintraub, S., \& Mesulam, M.-M. (1987). Right cerebral dominance in spatial attention. Further evidence based on ipsilateral neglect. Archives of Neurology, 44, 621-625.

Wickens, C. D. (1980). The structure of attentional resources. In R. S. Nickerson (Ed.), Attention and performance VIII (pp. 239-257). Hillsdale, NJ: Erlbaum.

Wickens, C. D. (1984). Processing resources in attention. In R. Parasuraman \& D. R. Davies (Eds.), Varieties of attention (pp. 63-102). New York, NY: Academic Press.

Wilson, F. A., Scalaidhe, S. P., \& Goldman-Rakic, P. S. (1993). Dissociation of object and spatial processing domains in primate prefrontal cortex. Science, 260, 1955-1958.

Wilson, H. R., \& Wilkinson, F. (2002). Symmetry perception: A novel approach for biological shapes. Vision Research, 42, 589-597.

Wolfe, J. M. (1994). Guided search 2.0: A revised model of visual search. Psychonomic Bulletin \& Review, 1, 202-238.

Womelsdorf, T., Schoffelen, J.-M., Oostenveld, R., Singer, W., Desimone, R., Engel, A. K., \& Fries, P. (2007). Modulation of neuronal interactions through neuronal synchronization. Science, 316, 1609-1612.

Woodman, G. F., \& Luck, S. J. (1999). Electrophysiological measurement of rapid shifts of attention during visual search. Nature, 400, 867-869.

Woodman, G. F., \& Luck, S. J. (2003). Serial deployment of attention during visual search. Journal of Experimental Psychology: Human Perception and Performance, 29, 121-138.

Wurtz, R. H., \& Goldberg, M. E. (1972). The primate superior colliculus and the shift of visual attention. Investigative Ophthalmology, 11, 441450.

Yantis, S. (1988). On analog movements of visual attention. Perception \& Psychophysics, 43, 203-206.

Yantis, S., \& Johnston, J. C. (1990). On the locus of visual selection: Evidence from focused attention tasks. Journal of Experimental Psychology: Human Perception and Performance, 16, 135-149.

Zimba, L. D., \& Hughes, H. C. (1987). Distractor-target interactions during directed visual attention. Spatial Vision, 2, 117-149.

Zuvic, S. M., Visser, T. A., \& Di Lollo, V. (2000). Direct estimates of processing delays in the attentional blink. Psychological Research, 63, 192-198.

Received June 24, 2008

Revision received October 22, 2009

Accepted October 23, 2009
Postscript: Split Spatial Attention? The Data Remain Difficult to Interpret

Bert Jans

Maastricht University

Judith C. Peters

Maastricht University and Netherlands Institute for Neuroscience, Amsterdam, the Netherlands

Peter De Weerd Maastricht University

A growing number of studies claim that spatial attention can be split "on demand" into several, segregated foci of enhanced processing. Intrigued by the theoretical ramifications of this proposal, we analyzed 19 relevant sets of experiments using four methodological criteria. We typically found several methodological limitations in each study that precluded convincing conclusions. Cave, Bush and Taylor (2010), however, find our criteria unnecessarily constraining and suggest that we have a theoretical bias that prevented us from considering valid evidence for divided spatial attention.

Divided spatial attention is defined as a temporally stable allocation of attentional resources over separate spatial locations permitting simultaneous processing of stimuli in multiple locations. We suggested that sufficiently difficult tasks (Criterion 1), briefly presented stimuli (Criterion 2), an appropriate cue-to-target interval (Criterion 3), and sufficiently dense probing of attention away from target locations (Criterion 4) increase the probability of a plausible conclusion of divided spatial attention. Contrary to our 